Supporting Infromation Table S2: Primary data

Flow cytometry

\begin{tabular}{|c|c|c|c|c|c|c|c|}
\hline locality & ID & date & index & 1/index & $2 \mathrm{C}$ & CV sample & CV std \\
\hline \multirow{7}{*}{ CZ, Beskydy Mts, Kladnatá hill } & BES 01 & 16.11 .2010 & 1,235 & 0,810 & 7,36 & 2,40 & 2,04 \\
\hline & BES 02 & 16.11 .2010 & 1,234 & 0,810 & 7,37 & 2,55 & 2,03 \\
\hline & BES 03 & 16.11 .2010 & 1,619 & 0,618 & 5,61 & 2,71 & 1,96 \\
\hline & BES 04 & 16.11 .2010 & 1,210 & 0,826 & 7,51 & 2,82 & 2,54 \\
\hline & BES 05 & 16.11 .2010 & 1,239 & 0,807 & 7,34 & 2,26 & 2,10 \\
\hline & BES 08 & 16.11 .2010 & 1,698 & 0,589 & 5,35 & 2,82 & 1,97 \\
\hline & BES 09 & 16.11 .2010 & 1,259 & 0,794 & 7,22 & 2,44 & 2,08 \\
\hline $\begin{array}{l}\text { CZ, Brdy, Hutě pod } \\
\text { Třemšínem, Kobylí hlava hill } \\
\end{array}$ & TRI+P & 13.7 .2010 & 1,801 & 0,555 & 5,05 & 3,50 & 2,56 \\
\hline \multirow[b]{2}{*}{ CZ, Brdy, Věšín } & $1+P$ & 11.5 .2010 & 1,220 & 0,820 & 7,45 & 4,84 & 2,57 \\
\hline & $2+P$ & 11.5 .2010 & 1,238 & 0,808 & 7,34 & 4,48 & 2,10 \\
\hline \multirow{10}{*}{$\begin{array}{l}\text { CZ, Bohemian forest, } \\
\text { Malinová hill }\end{array}$} & Malin.H.1+P & 14.7.2010 & 1,415 & 0,707 & 6,42 & 3,16 & 2,49 \\
\hline & Malin.H.2 & 14.7.2010 & 1,654 & 0,605 & 5,50 & 3,84 & 2,40 \\
\hline & Malin.H.3 & 14.7.2010 & 1,396 & 0,716 & 6,51 & 2,79 & 2,49 \\
\hline & Malin.H.4 & 14.7.2010 & 1,414 & 0,707 & 6,43 & 2,84 & 2,56 \\
\hline & Malin.H.5 & 14.7 .2010 & 1,241 & 0,806 & 7,32 & 2,85 & 2,05 \\
\hline & Malin.H.6 & 16.7.2010 & 1,400 & 0,714 & 6,49 & 2,58 & 2,53 \\
\hline & Malin.H.7 & 16.7.2010 & 1,426 & 0,701 & 6,37 & 3,35 & 2,84 \\
\hline & Malin.H.8 & 16.7.2010 & 1,408 & 0,710 & 6,46 & 2,76 & 2,55 \\
\hline & Malin.H.9 & 16.7.2010 & 1,430 & 0,699 & 6,36 & 4,27 & 2,87 \\
\hline & Malin.H.10 & 16.7.2010 & 1,423 & 0,703 & 6,39 & 3,81 & 2,62 \\
\hline \multirow{2}{*}{ CZ, Dolní Cerekev, Lysá hill } & BES 06 & 16.11 .2010 & 1,676 & 0,597 & 5,42 & 2,64 & 1,84 \\
\hline & BES 07 & 16.11.2010 & 1,663 & 0,601 & 5,47 & 2,67 & 2,51 \\
\hline \multirow{4}{*}{$\begin{array}{l}\text { CZ, Jeseníky Mts, Kamzičí } \\
\text { hill }\end{array}$} & Je $1 / 1+P$ & 27.9 .2010 & 1,184 & 0,845 & 7,68 & 3,32 & 1,87 \\
\hline & Je $1 / 2$ & 27.9.2010 & 1,195 & 0,837 & 7,61 & 3,18 & 2,43 \\
\hline & Je $1 / 3$ & 27.9 .2010 & 1,185 & 0,844 & 7,67 & 3,35 & 2,11 \\
\hline & Je $1 / 4$ & 27.9.2010 & 1,222 & 0,818 & 7,44 & 1,93 & 2,02 \\
\hline \multirow{5}{*}{$\begin{array}{l}\text { CZ, Jeseníky Mts, Praděd, } \\
\text { Peter's rocks }\end{array}$} & Je $3 / 1$ & 27.9.2010 & 1,188 & 0,842 & 7,65 & 3,29 & 2,35 \\
\hline & Je $3 / 2$ & 27.9.2010 & 1,228 & 0,814 & 7,40 & 2,37 & 2,25 \\
\hline & Je $3 / 3$ & 27.9 .2010 & 1,201 & 0,833 & 7,57 & 2,47 & 2,43 \\
\hline & Je $3 / 4$ & 27.9.2010 & 1,233 & 0,811 & 7,37 & 3,31 & 2,64 \\
\hline & Je $3 / 5$ & 27.9.2010 & 1,235 & 0,810 & 7,36 & 2,79 & 2,40 \\
\hline \multirow{2}{*}{$\begin{array}{l}\text { CZ, Jeseníky Mts, Praděd, } \\
\text { Table's rocks }\end{array}$} & Je $2 / 1$ & 27.9.2010 & 1,187 & 0,842 & 7,66 & 3,33 & 2,12 \\
\hline & Je $2 / 2$ & 27.9 .2010 & 1,210 & 0,826 & 7,51 & 3,02 & 1,99 \\
\hline \multirow{2}{*}{$\begin{array}{l}\text { CZ, Jeseníky Mts, Praděd, } \\
\text { Vysoká hole hill }\end{array}$} & Je $4 / 1$ & 27.9 .2010 & 1,214 & 0,824 & 7,49 & 2,57 & 2,03 \\
\hline & Je $4 / 2$ & 27.9.2010 & 1,213 & 0,824 & 7,49 & 2,49 & 2,05 \\
\hline & RAMZ 01 & 4.11 .2010 & 1,438 & 0,695 & 6,32 & 2,43 & 2,35 \\
\hline & RAMZ 02 & 4.11 .2010 & 1,794 & 0,557 & 5,07 & 2,41 & 2,39 \\
\hline & RAMZ 03 & 4.11 .2010 & 1,645 & 0,608 & 5,53 & 2,88 & 2,56 \\
\hline & RAMZ 04 & 4.11 .2010 & 1,221 & 0,819 & 7,44 & 2,09 & 2,53 \\
\hline & RAMZ 05 & 4.11 .2010 & 1,627 & 0,615 & 5,59 & 3,96 & 2,14 \\
\hline & RAMZ 06 & 4.11 .2010 & 1,662 & 0,602 & 5,47 & 2,81 & 2,89 \\
\hline & RAMZ 07 & 4.11 .2010 & 1,594 & 0,627 & 5,70 & 3,72 & 2,54 \\
\hline & RAMZ 08 & 4.11 .2010 & 1,827 & 0,547 & 4,98 & 3,19 & 2,47 \\
\hline & RAMZ 09 & 4.11 .2010 & 1,714 & 0,583 & 5,30 & 3,33 & 2,52 \\
\hline & RAMZ 10 & 4.11 .2010 & 1,417 & 0,706 & 6,41 & 3,14 & 2,58 \\
\hline
\end{tabular}




\begin{tabular}{|c|c|c|c|c|c|c|c|}
\hline & RAMZ 11 & 4.11 .2010 & 1,419 & 0,705 & 6,41 & 2,34 & 2,30 \\
\hline & RAMZ 12 & 4.11 .2010 & 1,447 & 0,691 & 6,28 & 2,66 & 2,22 \\
\hline & RAMZ 13 & 4.11 .2010 & 1,826 & 0,548 & 4,98 & 2,94 & 2,65 \\
\hline & $\mathrm{R} 1+\mathrm{P}$ & 23.10 .2009 & 1,651 & 0,606 & 5,51 & 2,64 & 1,84 \\
\hline & $R 2+P$ & 23.10 .2009 & 1,718 & 0,582 & 5,29 & 2,83 & 2,43 \\
\hline & $R 3+P$ & 23.10 .2009 & 1,658 & 0,603 & 5,48 & 2,95 & 2,15 \\
\hline & R4+P & 23.10 .2009 & 1,692 & 0,591 & 5,37 & 2,74 & 1,89 \\
\hline & R5+P & 23.10 .2009 & 1,833 & 0,546 & 4,96 & 2,79 & 2,06 \\
\hline & R6+P & 23.10 .2009 & 1,741 & 0,574 & 5,22 & 2,61 & 1,96 \\
\hline & $R 7+P$ & 23.10 .2009 & 1,686 & 0,593 & 5,39 & 2,47 & 1,51 \\
\hline & R8+P & 23.10 .2009 & 1,617 & 0,618 & 5,62 & 2,89 & 2,17 \\
\hline & R9+P & 23.10 .2009 & 1,249 & 0,801 & 7,28 & 2,81 & 2,27 \\
\hline & R10+P & 23.10 .2009 & 1,419 & 0,705 & 6,41 & 2,76 & 2,39 \\
\hline & R11+P & 23.10 .2009 & 1,650 & 0,606 & 5,51 & 2,73 & 2,16 \\
\hline & $R 12+P$ & 23.10 .2009 & 1,663 & 0,601 & 5,47 & 2,64 & 1,93 \\
\hline & $R 13+P$ & 23.10 .2009 & 1,742 & 0,574 & 5,22 & 2,78 & 2,31 \\
\hline & R14+P & 23.10 .2009 & 1,744 & 0,573 & 5,21 & 2,80 & 2,49 \\
\hline & R15+P & 23.10 .2009 & 1,647 & 0,607 & 5,52 & 2,70 & 2,58 \\
\hline CZ, Jeseníky Mts, Ramzová- & R16+P & 23.10 .2009 & 1,249 & 0,801 & 7,28 & 2,81 & 2,27 \\
\hline Černava ski slope & $R 17+P$ & 23.10 .2009 & 1,655 & 0,604 & 5,49 & 3,51 & 2,71 \\
\hline & R18+P & 23.10 .2009 & 1,782 & 0,561 & 5,10 & 2,73 & 2,61 \\
\hline & R19+P & 23.10 .2009 & 1,426 & 0,701 & 6,37 & 2,91 & 2,08 \\
\hline & $R 20+P$ & 23.10 .2009 & 1,675 & 0,597 & 5,43 & 2,81 & 2,26 \\
\hline & $R 21+P$ & 23.10 .2009 & 1,838 & 0,544 & 4,95 & 2,82 & 2,06 \\
\hline & $R 22+P$ & 23.10 .2009 & 1,264 & 0,791 & 7,19 & 2,88 & 2,34 \\
\hline & $R 23+P$ & 23.10 .2009 & 1,465 & 0,683 & 6,20 & 2,90 & 2,22 \\
\hline & R24+P & 23.10 .2009 & 1,693 & 0,591 & 5,37 & 2,39 & 2,40 \\
\hline & $R 25+P$ & 23.10 .2009 & 1,272 & 0,786 & 7,15 & 2,25 & 1,83 \\
\hline & $R 26+P$ & 23.10 .2009 & 1,243 & 0,805 & 7,31 & 2,35 & 2,49 \\
\hline & $R 27+P$ & 23.10 .2009 & 1,782 & 0,561 & 5,10 & 2,73 & 2,61 \\
\hline & $R 28+P$ & 23.10 .2009 & 1,596 & 0,627 & 5,70 & 3,95 & 2,75 \\
\hline & R29+P & 23.10 .2009 & 1,642 & 0,609 & 5,54 & 2,80 & 1,94 \\
\hline & R30+P & 23.10 .2009 & 1,643 & 0,609 & 5,53 & 2,86 & 2,13 \\
\hline & R31+P & 23.10 .2009 & 1,256 & 0,796 & 7,24 & 2,67 & 2,50 \\
\hline & $R 32+P$ & 23.10 .2009 & 1,680 & 0,595 & 5,41 & 2,68 & 2,34 \\
\hline & R33+P & 23.10.2009 & 1,671 & 0,598 & 5,44 & 2,85 & 2,35 \\
\hline & R34+P & 23.10 .2009 & 1,685 & 0,593 & 5,39 & 3,52 & 2,57 \\
\hline & R35+P & 23.10 .2009 & 1,442 & 0,693 & 6,30 & 2,78 & 2,58 \\
\hline & $R 36+P$ & 23.10 .2009 & 1,672 & 0,598 & 5,44 & 2,78 & 2,44 \\
\hline & R37+P & 23.10.2009 & 1,819 & 0,550 & 5,00 & 2,54 & 2,45 \\
\hline & R38+P & 26.10 .2009 & 1,720 & 0,581 & 5,28 & 2,70 & 2,34 \\
\hline & R39+P & 26.10 .2009 & 1,414 & 0,707 & 6,43 & 2,48 & 1,79 \\
\hline & R40+P & 26.10 .2009 & 1,629 & 0,614 & 5,58 & 2,59 & 1,76 \\
\hline & R41+P & 26.10 .2009 & 1,230 & 0,813 & 7,39 & 2,39 & 1,91 \\
\hline & $R 42+P$ & 26.10.2009 & 1,814 & 0,551 & 5,01 & 2,59 & 2,20 \\
\hline & $R 43+P$ & 26.10 .2009 & 1,695 & 0,590 & 5,36 & 2,99 & 1,99 \\
\hline & R44+P & 26.10 .2009 & 1,613 & 0,620 & 5,64 & 2,74 & 1,92 \\
\hline & R45+P & 26.10 .2009 & 1,727 & 0,579 & 5,26 & 2,71 & 1,85 \\
\hline & Je $6 / 1$ & 27.9 .2010 & 1,216 & 0,822 & 7,48 & 3,88 & 1,79 \\
\hline & Je $6 / 1 a$ & 27.9.2010 & 1,267 & 0,789 & 7,17 & 2,77 & 2,78 \\
\hline
\end{tabular}




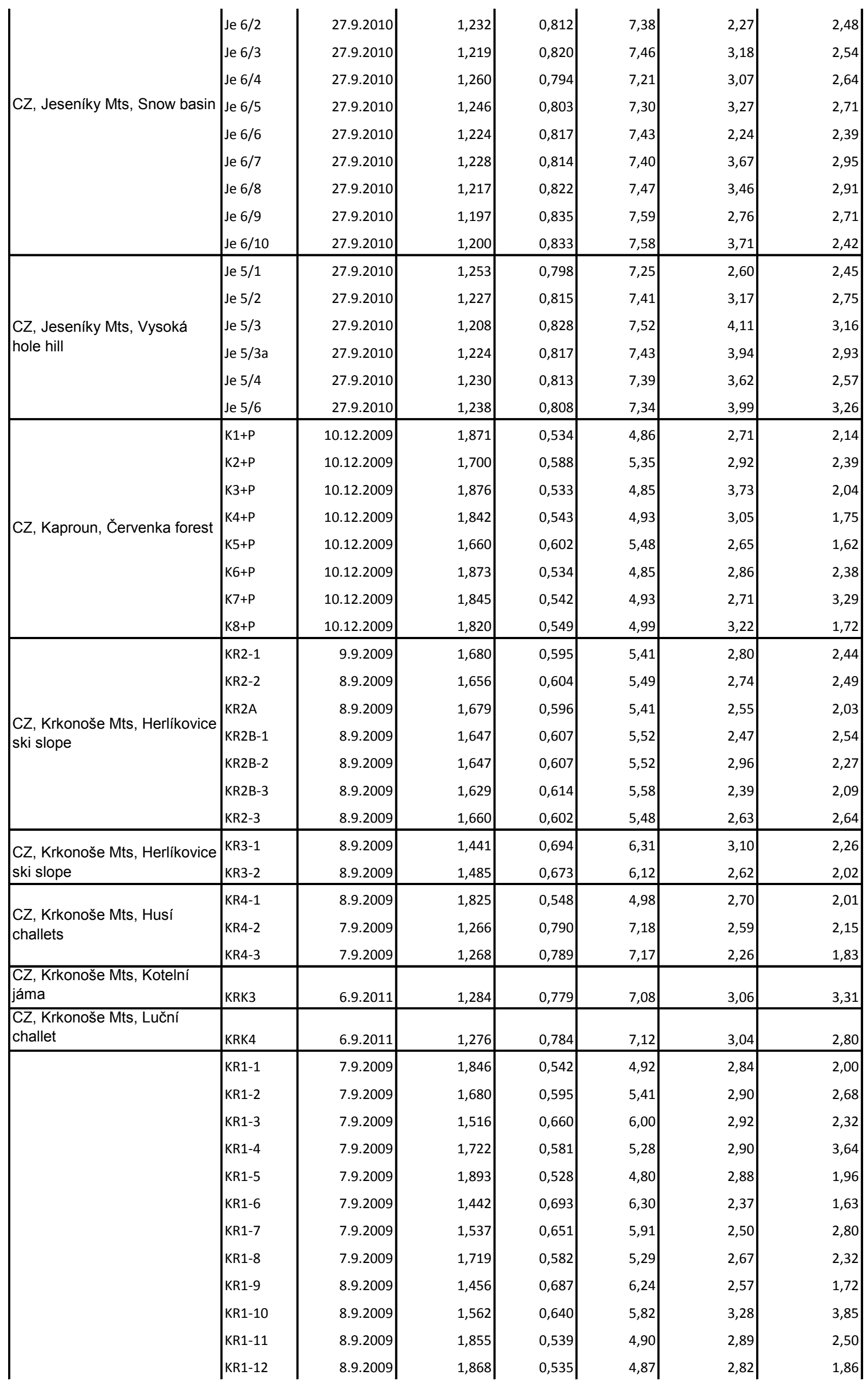




\begin{tabular}{|c|c|c|c|c|c|c|c|}
\hline & KR1-13 & 8.9 .2009 & 1,723 & 0,580 & 5,28 & 2,76 & 2,85 \\
\hline & KR1-14 & 8.9.2009 & 1,851 & 0,540 & 4,91 & 3,53 & 2,23 \\
\hline & KR1-15 & 8.9 .2009 & 1,506 & 0,664 & 6,04 & 2,75 & 2,49 \\
\hline & KR1-16 & 8.9 .2009 & 1,866 & 0,536 & 4,87 & 2,63 & 1,84 \\
\hline & KR1-17 & 7.9 .2009 & 1,522 & 0,657 & 5,97 & 2,39 & 2,51 \\
\hline & KR1-18 & 7.9.2009 & 1,290 & 0,775 & 7,05 & 2,15 & 2,11 \\
\hline & KR1-19 & 7.9.2009 & 1,880 & 0,532 & 4,84 & 3,52 & 2,68 \\
\hline & KR1-20 & 7.9.2009 & 1,860 & 0,538 & 4,89 & 3,39 & 2,43 \\
\hline & KR1-21 & 9.9 .2009 & 1,276 & 0,784 & 7,12 & 2,37 & 1,64 \\
\hline $\begin{array}{l}\text { LL, nikomuse IVIS, IVIedvedin } \\
\text { ski slope }\end{array}$ & KR1-22 & 9.9 .2009 & 1,476 & 0,678 & 6,16 & 1,67 & 2,61 \\
\hline & KR1-23 & 8.9 .2009 & 1,527 & 0,655 & 5,95 & 2,78 & 2,00 \\
\hline & KR1-24 & 8.9 .2009 & 1,497 & 0,668 & 6,07 & 2,68 & 1,88 \\
\hline & KR1-25 & 8.9 .2009 & 1,800 & 0,556 & 5,05 & 2,23 & 1,56 \\
\hline & KR1-26 & 8.9.2009 & 1,453 & 0,688 & 6,26 & 2,80 & 2,44 \\
\hline & KR1-27 & 8.9 .2009 & 1,512 & 0,661 & 6,01 & 3,32 & 3,09 \\
\hline & KR1-28 & 8.9 .2009 & 1,869 & 0,535 & 4,86 & 3,15 & 2,45 \\
\hline & KR1-29 & 8.9 .2009 & 1,651 & 0,606 & 5,51 & 2,91 & 2,12 \\
\hline & KR1-30 & 8.9 .2009 & 1,816 & 0,551 & 5,01 & 3,30 & 2,52 \\
\hline & KR1-31 & 8.9 .2009 & 1,645 & 0,608 & 5,53 & 2,25 & 2,06 \\
\hline & KR1-32 & 8.9.2009 & 1,482 & 0,675 & 6,13 & 2,86 & 2,66 \\
\hline & KR1-33 & 8.9.2009 & 1,500 & 0,667 & 6,06 & 2,57 & 1,56 \\
\hline & KR1-34 & 9.9.2009 & 1,506 & 0,664 & 6,04 & 2,71 & 2,26 \\
\hline & KRK1 -1 & 6.9 .2011 & 1,562 & 0,640 & 5,82 & 3,58 & 3,18 \\
\hline & & 2.11.2011 & 1,490 & 0,671 & 6,10 & 2,63 & 2,67 \\
\hline & & 3.11 .2011 & 1,540 & 0,649 & 5,90 & 2,64 & 2,45 \\
\hline & KRK1 -2 & 1.11 .2011 & 1,774 & 0,564 & 5,12 & 3,63 & 3,05 \\
\hline & & 2.11 .2011 & 1,856 & 0,539 & 4,90 & 2,97 & 2,62 \\
\hline & & 3.11 .2011 & 1,837 & 0,544 & 4,95 & 3,07 & 2,39 \\
\hline & KRK1 -3 & 1.11 .2011 & 1,496 & 0,668 & 6,08 & 3,43 & 3,14 \\
\hline & & 2.11 .2011 & 1,508 & 0,663 & 6,03 & 3,09 & 1,48 \\
\hline & & 3.11 .2011 & 1,490 & 0,671 & 6,10 & 2,77 & 2,83 \\
\hline & $1+P$ & 7.7.2010 & 1,248 & 0,801 & 7,28 & 2,20 & 2,06 \\
\hline & $2+P$ & 8.7.2010 & 1,233 & 0,811 & 7,37 & 2,37 & 2,52 \\
\hline lcZ Krušné Mts Boží Dar & $3+P$ & 8.7 .2010 & 1,243 & 0,805 & 7,31 & 2,35 & 2,42 \\
\hline 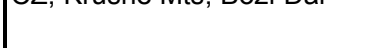 & $4+P$ & 8.7.2010 & 1,248 & 0,801 & 7,28 & 2,78 & 2,71 \\
\hline & $5+P$ & 8.7 .2010 & 1,236 & 0,809 & 7,35 & 2,71 & 2,13 \\
\hline & $6+P$ & 8.7.2010 & 1,237 & 0,808 & 7,35 & 3,09 & 2,10 \\
\hline & $1+P$ & 7.7.2010 & 1,442 & 0,693 & 6,30 & 3,08 & 2,15 \\
\hline CZ, Krušné Mts, Horní Blatná & $2+P$ & 7.7.2010 & 1,415 & 0,707 & 6,42 & 3,44 & 2,28 \\
\hline & $3+P$ & 7.7.2010 & 1,424 & 0,702 & 6,38 & 3,67 & 2,69 \\
\hline & KAL 1 & 5.9 .2011 & 1,660 & 0,602 & 5,48 & 2,34 & 2,07 \\
\hline & & 1.11 .2011 & 1,666 & 0,600 & 5,46 & 3,25 & 1,83 \\
\hline CZ, Krušné Mts, Kalek & & 3.11 .2011 & 1,700 & 0,588 & 5,35 & 2,87 & 2,85 \\
\hline & KAL 2 & 5.9 .2011 & 1,680 & 0,595 & 5,41 & 3,03 & 2,10 \\
\hline & & 1.11 .2011 & 1,693 & 0,591 & 5,37 & 2,68 & 2,35 \\
\hline & & 3.11 .2011 & 1,683 & 0,594 & 5,40 & 2,96 & 2,50 \\
\hline & RYŽ 1 & 31.10 .2011 & 1,676 & 0,597 & 5,42 & 2,78 & 2,15 \\
\hline & & 1.11.2011 & 1,673 & 0,598 & 5,43 & 2,93 & 2,50 \\
\hline & & 3.11 .2011 & 1,682 & 0,595 & 5,40 & 2,85 & 2,50 \\
\hline & RYŽ 2 & 5.9.2011 & 1,266 & 0,790 & 7,18 & 2,73 & \\
\hline
\end{tabular}




\begin{tabular}{|c|c|c|c|c|c|c|c|}
\hline \multirow[t]{5}{*}{ CZ, Krušné Mts, Ryžovna } & \multirow{5}{*}{ RYŽ 3} & 1.11 .2011 & 1,267 & 0,789 & 7,17 & 2,97 & 2,51 \\
\hline & & 3.11 .2011 & 1,297 & 0,771 & 7,01 & 2,23 & 1,64 \\
\hline & & 5.9 .2011 & 1,254 & 0,797 & 7,25 & 2,11 & 2,48 \\
\hline & & 1.11 .2011 & 1,261 & 0,793 & 7,21 & 2,99 & 2,62 \\
\hline & & 3.11 .2011 & 1,273 & 0,786 & 7,14 & 2,83 & 2,44 \\
\hline \multirow{9}{*}{ CZ, Krušné Mts, Výsluní } & \multirow[t]{3}{*}{ VÝSL 1} & 5.9.2011 & 1,694 & 0,590 & 5,37 & 2,86 & 2,36 \\
\hline & & 1.11 .2011 & 1,703 & 0,587 & 5,34 & 2,57 & 2,12 \\
\hline & & 2.11 .2011 & 1,725 & 0,580 & 5,27 & 2,95 & 1,77 \\
\hline & \multirow[t]{3}{*}{ VÝSL 2} & 5.9.2011 & 1,673 & 0,598 & 5,43 & 2,87 & 2,19 \\
\hline & & 1.11 .2011 & 1,681 & 0,595 & 5,41 & 2,71 & 2,11 \\
\hline & & 3.11 .2011 & 1,672 & 0,598 & 5,44 & 3,20 & 2,35 \\
\hline & \multirow[t]{3}{*}{ VÝSL 3} & 1.11 .2011 & 1,693 & 0,591 & 5,37 & 2,82 & 2,44 \\
\hline & & 2.11 .2011 & 1,695 & 0,590 & 5,36 & 2,72 & 2,86 \\
\hline & & 3.11.2011 & 1,702 & 0,588 & 5,34 & 2,82 & 2,24 \\
\hline \multirow{11}{*}{$\begin{array}{l}\text { CZ, Orlické Mts, Sedloňov, } \\
\text { Polom hill }\end{array}$} & POL 1+P & 2.8 .2010 & 1,795 & 0,557 & 5,06 & 2,88 & 1,93 \\
\hline & POL 2 & 2.8 .2010 & 1,826 & 0,548 & 4,98 & 3,19 & 2,36 \\
\hline & POL 3 & 2.8 .2010 & 1,794 & 0,557 & 5,07 & 3,49 & 2,95 \\
\hline & POL 4 & 2.8 .2010 & 1,822 & 0,549 & 4,99 & 2,82 & 2,80 \\
\hline & POL 5 & 2.8 .2010 & 1,847 & 0,541 & 4,92 & 3,90 & 2,61 \\
\hline & POL 5 & 4.11 .2010 & 1,825 & 0,548 & 4,98 & 3,63 & 2,37 \\
\hline & POL 5 & 3.3.2011 & 1,847 & 0,541 & 4,92 & 1,88 & 1,71 \\
\hline & POL 6 & 2.8 .2010 & 1,828 & 0,547 & 4,97 & 4,05 & 2,45 \\
\hline & POL 7 & 2.8 .2010 & 1,743 & 0,574 & 5,22 & 3,82 & 3,11 \\
\hline & POL 8 & 2.8 .2010 & 1,830 & 0,546 & 4,97 & 4,41 & 3,27 \\
\hline & POL 9 & 2.8 .2010 & 1,826 & 0,548 & 4,98 & 4,05 & 2,86 \\
\hline \multirow{5}{*}{$\begin{array}{l}\text { CZ, Radhošt'ské Beskydy Mts, } \\
\text { Krásná hill }\end{array}$} & BESK1 & 8.12 .2010 & 1,660 & 0,602 & 5,48 & 2,25 & 1,78 \\
\hline & BESK2 & 8.12 .2010 & 1,675 & 0,597 & 5,43 & 2,89 & 2,25 \\
\hline & BESK3 & 8.12 .2010 & 1,595 & 0,627 & 5,70 & 3,60 & 2,05 \\
\hline & BESK4 & 8.12 .2010 & 1,669 & 0,599 & 5,45 & 2,37 & 1,74 \\
\hline & BESK5 & 8.12 .2010 & 1,702 & 0,588 & 5,34 & 2,18 & 1,82 \\
\hline \multirow{5}{*}{ CZ, Sklené nad Oslavou } & SK1+P & 26.10 .2009 & 1,658 & 0,603 & 5,48 & 2,50 & 2,10 \\
\hline & SK1 & 10.12.2009 & 1,688 & 0,592 & 5,39 & 3,41 & 2,08 \\
\hline & SK2+P & 26.10 .2009 & 1,667 & 0,600 & 5,45 & 2,92 & 2,09 \\
\hline & SK3+P & 26.10.2009 & 1,638 & 0,611 & 5,55 & 2,32 & 1,95 \\
\hline & SK3 & 10.12 .2009 & 1,656 & 0,604 & 5,49 & 2,67 & 1,76 \\
\hline \multirow{15}{*}{$\begin{array}{l}\text { CZ, Slavkovský les, Bečov } \\
\text { nad Teplou }\end{array}$} & BEČ $1+P$ & 14.9 .2010 & 1,697 & 0,589 & 5,36 & 3,62 & 2,11 \\
\hline & BEČ 2 & 14.9 .2010 & 1,679 & 0,596 & 5,41 & 4,65 & 2,92 \\
\hline & BEČ 3 & 14.9 .2010 & 1,675 & 0,597 & 5,43 & 4,29 & 2,34 \\
\hline & BEČ 4 & 14.9 .2010 & 1,648 & 0,607 & 5,52 & 3,74 & 2,97 \\
\hline & BEČ 5 & 14.9 .2010 & 1,667 & 0,600 & 5,45 & 4,74 & 2,71 \\
\hline & BEČ 6 & 14.9 .2010 & 1,421 & 0,704 & 6,40 & 3,99 & 2,33 \\
\hline & BEČ 7 & 14.9 .2010 & 1,415 & 0,707 & 6,42 & 2,69 & 2,57 \\
\hline & BEČ 8 & 14.9 .2010 & 1,688 & 0,592 & 5,39 & 4,87 & 2,85 \\
\hline & BEČ 9 & 14.9 .2010 & 1,627 & 0,615 & 5,59 & 3,49 & 2,40 \\
\hline & BEČ 10 & 14.9 .2010 & 1,632 & 0,613 & 5,57 & 3,87 & 2,91 \\
\hline & BEČ 11 & 14.9 .2010 & 1,631 & 0,613 & 5,57 & 4,96 & 3,54 \\
\hline & BEČ 12 & 14.9.2010 & 1,618 & 0,618 & 5,62 & 3,76 & 2,90 \\
\hline & BEČ 13 & 14.9 .2010 & 1,654 & 0,605 & 5,50 & 3,64 & 2,90 \\
\hline & $B \cup C 01+P$ & 10.9 .2010 & 1,244 & 0,804 & 7,31 & 4,80 & 4, \\
\hline & BUC 02 & 10.9.2010 & 1,292 & 0,774 & 7,04 & 3,85 & 2,66 \\
\hline
\end{tabular}




\begin{tabular}{|c|c|c|c|c|c|c|c|}
\hline \multirow{14}{*}{ CZ, Šumava, Bučina } & BUC 03 & 10.9 .2010 & 1,299 & 0,770 & 7,00 & 3,63 & 3,40 \\
\hline & BUC 04 & 10.9 .2010 & 1,266 & 0,790 & 7,18 & 3,39 & 3,59 \\
\hline & BUC 05 & 10.9 .2010 & 1,272 & 0,786 & 7,15 & 3,57 & 3,68 \\
\hline & BUC 06 & 10.9 .2010 & 1,257 & 0,796 & 7,23 & 3,49 & 3,23 \\
\hline & BUC 07 & 10.9 .2010 & 1,294 & 0,773 & 7,02 & 2,89 & 3,01 \\
\hline & BUC 08 & 10.9 .2010 & 1,305 & 0,766 & 6,97 & 2,18 & 3,72 \\
\hline & BUC 09 & 10.9 .2010 & 1,315 & 0,760 & 6,91 & 2,43 & 2,73 \\
\hline & BUC 1 & 18.10.2011 & 1,357 & 0,737 & 6,70 & 2,64 & 1,29 \\
\hline & BUC 2 & 18.10 .2011 & 1,296 & 0,772 & 7,01 & 2,56 & 1,64 \\
\hline & BUC 3 & 18.10 .2011 & 1,242 & 0,805 & 7,32 & 2,49 & 2,25 \\
\hline & BUC 4 & 18.10.2011 & 1,273 & 0,786 & 7,14 & 2,54 & 2,15 \\
\hline & BUC 5 & 18.10 .2011 & 1,413 & 0,708 & 6,43 & 3,03 & 2,59 \\
\hline & BUC 6 & 18.10 .2011 & 1,247 & 0,802 & 7,29 & 2,76 & 2,43 \\
\hline & BUC 7 & 18.10.2011 & 1,382 & 0,724 & 6,58 & 2,38 & 2,27 \\
\hline \multirow{6}{*}{ CZ, Šumava, Český Rudolec } & CRU 01 & 22.6 .2010 & 1,640 & 0,610 & 5,54 & 3,70 & 2,01 \\
\hline & CRU 02 & 22.6 .2010 & 1,648 & 0,607 & 5,52 & 4,54 & 1,98 \\
\hline & CRU 03 & 22.6 .2010 & 1,631 & 0,613 & 5,57 & 5,78 & 2,35 \\
\hline & CRU 04 & 22.6 .2010 & 1,662 & 0,602 & 5,47 & 3,80 & 2,37 \\
\hline & CRU 05 & 22.6 .2010 & 1,620 & 0,617 & 5,61 & 4,90 & 2,47 \\
\hline & CRU 06 & 22.6 .2010 & 1,598 & 0,626 & 5,69 & 5,22 & 2,80 \\
\hline \multirow{3}{*}{ CZ, Šumava, Hůrka } & HUR 01+P & 9.9 .2010 & 1,266 & 0,790 & 7,18 & 3,99 & 3,54 \\
\hline & HUR 02 & 9.9 .2010 & 1,326 & 0,754 & 6,86 & 4,55 & 3,46 \\
\hline & HUR 03 & 9.9 .2010 & 1,239 & 0,807 & 7,34 & 5,06 & 4,08 \\
\hline & CBS 01A & 9.7 .2010 & 1,384 & 0,723 & 6,57 & 3,55 & 2,17 \\
\hline & CBS 01B & 9.7 .2010 & 1,431 & 0,699 & 6,35 & 2,61 & 2,96 \\
\hline & CBS 02 & 9.7 .2010 & 1,253 & 0,798 & 7,25 & 2,66 & 2,42 \\
\hline & CBS 03 & 9.7 .2010 & 1,254 & 0,797 & 7,25 & 2,58 & 1,74 \\
\hline & CBS 04 & 9.7 .2010 & 1,399 & 0,715 & 6,50 & 2,63 & 1,97 \\
\hline & CBS 05 & 9.7 .2010 & 1,657 & 0,604 & 5,49 & 2,75 & 1,98 \\
\hline & CBS 06 & 9.7 .2010 & 1,651 & 0,606 & 5,51 & 3,39 & 2,26 \\
\hline & CBS 07 & 9.7 .2010 & 1,437 & 0,696 & 6,33 & 2,47 & 2,01 \\
\hline & CBS 08 & 9.7 .2010 & 1,264 & 0,791 & 7,19 & 2,36 & 2,31 \\
\hline & CBS 09 & 9.7 .2010 & 1,254 & 0,797 & 7,25 & 2,86 & 1,95 \\
\hline & CBS 10 & 9.7 .2010 & 1,635 & 0,612 & 5,56 & 3,06 & 2,06 \\
\hline & ZAD 2-1 & 18.10 .2011 & 1,411 & 0,709 & 6,44 & 2,42 & 2,04 \\
\hline & & 24.10.2011 & 1,441 & 0,694 & 6,31 & 2,52 & 2,31 \\
\hline & & 2.11.2011 & 1,422 & 0,703 & 6,39 & 2,55 & 1,60 \\
\hline & ZAD 2-2 & 24.10 .2011 & 1,455 & 0,687 & 6,25 & 2,21 & 1,86 \\
\hline & & 31.10 .2011 & 1,435 & 0,697 & 6,33 & 2,12 & 2,10 \\
\hline & & 2.11 .2011 & 1,455 & 0,687 & 6,25 & 2,78 & 2,17 \\
\hline & ZAD 2-3 & 18.10.2011 & 1,259 & 0,794 & 7,22 & 2,38 & 2,15 \\
\hline & & 31.10 .2011 & 1,256 & 0,796 & 7,24 & 2,26 & 2,11 \\
\hline & & 2.11 .2011 & 1,279 & 0,782 & 7,11 & 2,81 & 2,99 \\
\hline & ZAD 2-4 & 18.10.2011 & 1,388 & 0,720 & 6,55 & 2,36 & 2,06 \\
\hline & & 31.10 .2011 & 1,418 & 0,705 & 6,41 & 2,68 & 2,73 \\
\hline & & 2.11 .2011 & 1,403 & 0,713 & 6,48 & 2,93 & 2,85 \\
\hline & ZAD 2-5 & 18.10 .2011 & 1,429 & 0,700 & 6,36 & 2,89 & 2,72 \\
\hline & & 24.10.2011 & 1,435 & 0,697 & 6,33 & 2,27 & 1,94 \\
\hline & & 2.11.2011 & 1,430 & 0,699 & 6,36 & 2,74 & 2,23 \\
\hline & ZAD 2-6 & 18.10 .2011 & 1,573 & 0,636 & 5,78 & 1,66 & 2,16 \\
\hline
\end{tabular}




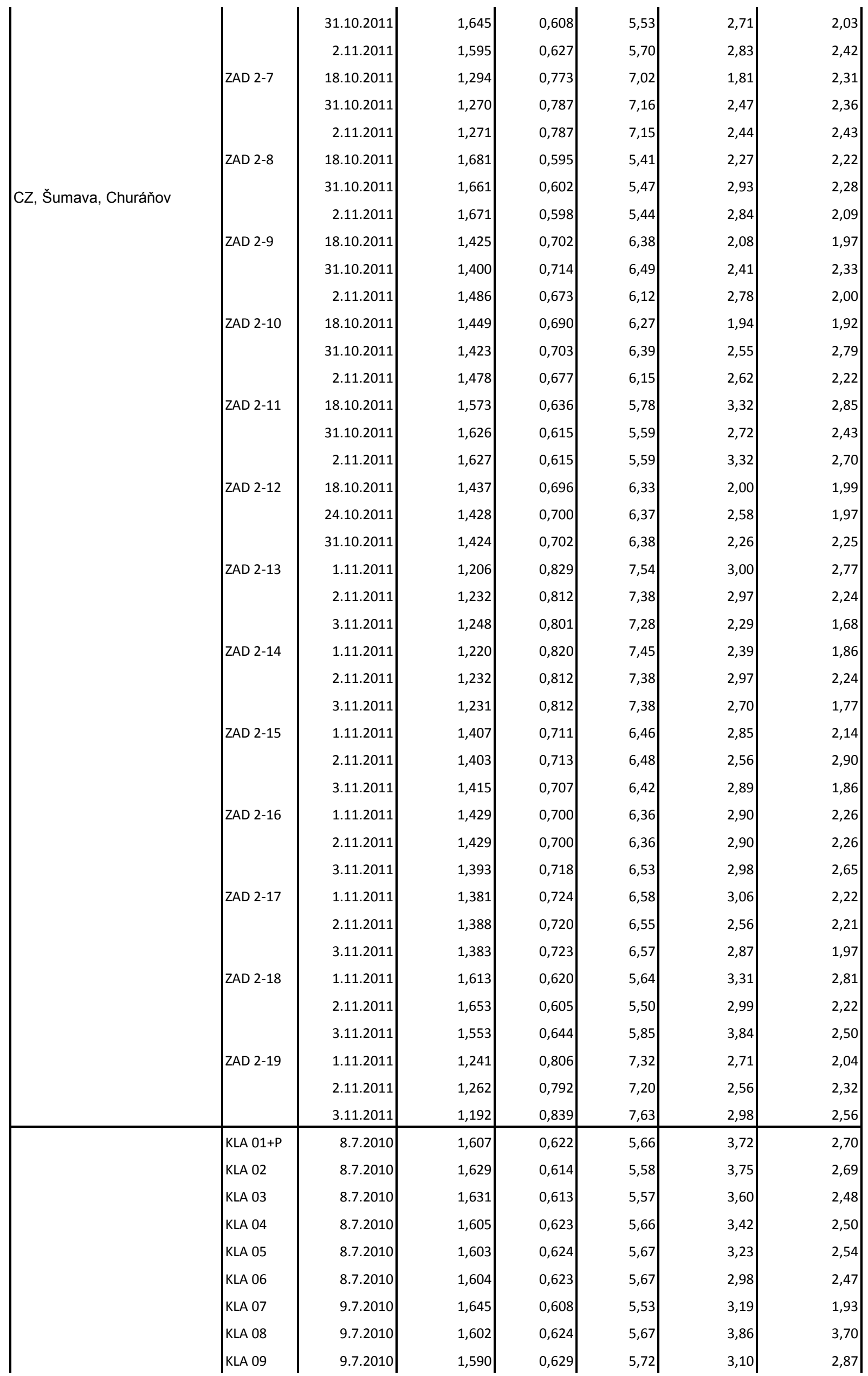




\begin{tabular}{|c|c|c|c|c|c|c|c|}
\hline & KLA 10 & 9.7.2010 & 1,624 & 0,616 & 5,60 & 3,18 & 2,50 \\
\hline & KLA 1 & 18.10 .2011 & 1,650 & 0,606 & 5,51 & 2,78 & 1,86 \\
\hline & & 31.10 .2011 & 1,611 & 0,621 & 5,64 & 2,55 & 2,09 \\
\hline CZ, Šumava, Klášterec & & 2.11 .2011 & 1,650 & 0,606 & 5,51 & 3,06 & 2,59 \\
\hline & KLA 2 & 18.10 .2011 & 1,603 & 0,624 & 5,67 & 3,27 & 2,16 \\
\hline & & 31.10 .2011 & 1,602 & 0,624 & 5,67 & 2,94 & 2,98 \\
\hline & & 2.11.2011 & 1,624 & 0,616 & 5,60 & 2,73 & 2,38 \\
\hline & KLA 3 & 18.10 .2011 & 1,641 & 0,609 & 5,54 & 2,85 & 2,31 \\
\hline & & 31.10 .2011 & 1,611 & 0,621 & 5,64 & 3,13 & 3,02 \\
\hline & & 2.11.2011 & 1,648 & 0,607 & 5,52 & 2,54 & 2,79 \\
\hline & KLA 4 & 24.10 .2011 & 1,621 & 0,617 & 5,61 & 2,08 & 2,21 \\
\hline & & 31.10 .2011 & 1,618 & 0,618 & 5,62 & 2,41 & 2,82 \\
\hline & & 2.11.2011 & 1,643 & 0,609 & 5,53 & 2,92 & 2,62 \\
\hline & KLA 5 & 24.10 .2011 & 1,626 & 0,615 & 5,59 & 2,42 & 2,03 \\
\hline & & 31.10 .2011 & 1,672 & 0,598 & 5,44 & 3,04 & 2,65 \\
\hline & & 2.11.2011 & 1,653 & 0,605 & 5,50 & 3,42 & 3,46 \\
\hline & KNI 01+P & 9.7.2010 & 1,427 & 0,701 & 6,37 & 2,88 & 2,18 \\
\hline & KNI 02 & 9.7 .2010 & 1,264 & 0,791 & 7,19 & 3,00 & 2,56 \\
\hline & KNI 03 & 9.7 .2010 & 1,712 & 0,584 & 5,31 & 3,24 & 2,38 \\
\hline & KNI 04 & 9.7 .2010 & 1,802 & 0,555 & 5,04 & 3,11 & 2,28 \\
\hline C7 Šumava Knížecí nláně & KNI 05 & 9.7 .2010 & 1,813 & 0,552 & 5,01 & 3,10 & 2,49 \\
\hline & KNI 06 & 9.7 .2010 & 1,809 & 0,553 & 5,02 & 3,56 & 2,92 \\
\hline & KNI 07 & 9.7 .2010 & 1,264 & 0,791 & 7,19 & 2,29 & 2,26 \\
\hline & KNI 08 & 9.7 .2010 & 1,254 & 0,797 & 7,25 & 2,39 & 2,07 \\
\hline & KNI 09 & 9.7 .2010 & 1,231 & 0,812 & 7,38 & 2,52 & 2,26 \\
\hline & KNI 10 & 9.7 .2010 & 1,241 & 0,806 & 7,32 & 2,40 & 2,14 \\
\hline & KTI 01+P & 12.7 .2010 & 1,686 & 0,593 & 5,39 & 3,02 & 2,93 \\
\hline CZ, Šumava, Ktiš & KTI 02 & 12.7 .2010 & 1,688 & 0,592 & 5,39 & 5,21 & 3,73 \\
\hline & KTI 03 & 12.7 .2010 & 1,720 & 0,581 & 5,28 & 4,75 & 3,73 \\
\hline & KUB 01 & 1.6 .2010 & 1,858 & 0,538 & 4,89 & 3,19 & 2,49 \\
\hline & KUB 02 & 2.6.2010 & 1,762 & 0,568 & 5,16 & 3,99 & 3,29 \\
\hline & KUB 03 & 2.6 .2010 & 1,265 & 0,791 & 7,19 & 4,09 & 3,01 \\
\hline & KUB 04 & 2.6.2010 & 1,240 & 0,806 & 7,33 & 4,79 & 4,18 \\
\hline & KUB 05 & 2.6.2010 & 1,416 & 0,706 & 6,42 & 5,10 & 3,51 \\
\hline & KUB 06 & 1.6.2010 & 1,270 & 0,787 & 7,16 & 3,21 & 2,65 \\
\hline & KUB 07 & 2.6.2010 & 1,427 & 0,701 & 6,37 & 3,83 & 3,31 \\
\hline & KUB 08/1 & 2.6 .2010 & 1,698 & 0,589 & 5,35 & 3,81 & 2,80 \\
\hline & KUB 08/2 & 2.6.2010 & 1,840 & 0,543 & 4,94 & 3,61 & 2,89 \\
\hline CZ, Šumava, Kubova hut' & KUB 08/3 & 1.6 .2010 & 1,684 & 0,594 & 5,40 & 2,59 & 2,04 \\
\hline & KUB 09 & 1.6 .2010 & 1,659 & 0,603 & 5,48 & 3,46 & 2,60 \\
\hline & KUB 10 & 1.6 .2010 & 1,274 & 0,785 & 7,14 & 2,77 & 2,42 \\
\hline & KUB 11 & 12.7 .2010 & 1,257 & 0,796 & 7,23 & 1,70 & 2,51 \\
\hline & KUB 12 & 12.7 .2010 & 1,250 & 0,800 & 7,27 & 2,00 & 2,71 \\
\hline & KUB 13 & 12.7 .2010 & 1,435 & 0,697 & 6,33 & 3,27 & 2,19 \\
\hline & KUB 14a & 12.7 .2010 & 1,443 & 0,693 & 6,30 & 2,87 & 3,12 \\
\hline & KUB 14b & 12.7 .2010 & 1,446 & 0,692 & 6,29 & 2,28 & 2,65 \\
\hline & KUB15+P20 & 12.7 .2010 & 1,717 & 0,582 & 5,29 & 2,71 & 2,11 \\
\hline & KUB16 & 12.7.2010 & 1,415 & 0,707 & 6,42 & 2,88 & 2,22 \\
\hline & OLS 01+P & 13.9 .2010 & 1,242 & 0,805 & 7,32 & 2,82 & 2,84 \\
\hline & OLS 02 & 13.9 .2010 & & 0,813 & 7,39 & 2,67 & 2,70 \\
\hline
\end{tabular}




\begin{tabular}{|c|c|c|c|c|c|c|c|}
\hline & OLS 03 & 13.9 .2010 & 1,262 & 0,792 & 7,20 & 2,87 & 2,90 \\
\hline & OLS 04 & 13.9 .2010 & 1,328 & 0,753 & 6,84 & 2,74 & 2,20 \\
\hline & OLS 05 & 13.9 .2010 & 1,242 & 0,805 & 7,32 & 2,09 & 2,94 \\
\hline & OLS 06 & 13.9 .2010 & 1,248 & 0,801 & 7,28 & 2,46 & 1,96 \\
\hline & OLS 07 & 13.9 .2010 & 1,278 & 0,782 & 7,11 & 2,40 & 2,75 \\
\hline & OLS 08 & 13.9.2010 & 1,254 & 0,797 & 7,25 & 2,46 & 2,83 \\
\hline & OLS 09 & 13.9.2010 & 1,254 & 0,797 & 7,25 & 2,66 & 3,25 \\
\hline CZ, Šumava, Kvilda - Olšinka & OLS 10 & 13.9 .2010 & 1,244 & 0,804 & 7,31 & 2,75 & 2,69 \\
\hline & OLS 11 & 13.9.2010 & 1,433 & 0,698 & 6,34 & 2,70 & 2,82 \\
\hline & OLS 12 & 13.9.2010 & 1,412 & 0,708 & 6,44 & 3,47 & 3,33 \\
\hline & OLS 13 & 14.9 .2010 & 1,424 & 0,702 & 6,38 & 2,45 & 2,33 \\
\hline & OLS 14 & 14.9 .2010 & 1,242 & 0,805 & 7,32 & 2,17 & 2,82 \\
\hline & OLS 15 & 14.9 .2010 & 1,226 & 0,816 & 7,41 & 2,10 & 2,65 \\
\hline & OLS 16 & 14.9 .2010 & 1,236 & 0,809 & 7,35 & 2,34 & 2,93 \\
\hline & OLS 17 & 14.9 .2010 & 1,228 & 0,814 & 7,40 & 2,23 & 3,01 \\
\hline & OLS 18 & 14.9 .2010 & 1,237 & 0,808 & 7,35 & 2,55 & 3,02 \\
\hline & OLS 19 & 14.9.2010 & 1,230 & 0,813 & 7,39 & 2,61 & 2,71 \\
\hline & TET 01+P & 9.9 .2010 & 1,235 & 0,810 & 7,36 & 2,36 & 2,91 \\
\hline & TET 01 & 4.11 .2010 & 1,267 & 0,789 & 7,17 & 2,42 & 2,50 \\
\hline & TET 01 & 3.3.2011 & 1,266 & 0,790 & 7,18 & 1,82 & 1,68 \\
\hline & TET 02 & 9.9 .2010 & 1,257 & 0,796 & 7,23 & 3,16 & 1,62 \\
\hline CZ, Šumava, Kvilda, Tetřev & TET 03 & 9.9 .2010 & 1,255 & 0,797 & 7,24 & 3,45 & 2,90 \\
\hline & TET 04 & 9.9 .2010 & 1,247 & 0,802 & 7,29 & 3,43 & 3,26 \\
\hline & KVIL & 18.10 .2011 & 1,211 & 0,826 & 7,51 & 2,09 & 2,70 \\
\hline & & 26.10 .2011 & 1,234 & 0,810 & 7,37 & 2,78 & 2,24 \\
\hline & & 31.10 .2011 & 1,214 & 0,824 & 7,49 & 3,01 & 2,32 \\
\hline & LAK 01+P & 10.9 .2010 & 1,265 & 0,791 & 7,19 & 3,19 & 2,64 \\
\hline & LAK 02 & 10.9.2010 & 1,249 & 0,801 & 7,28 & 3,05 & 2,66 \\
\hline C7 Šumava I aka lake & LAK 03 & 10.9 .2010 & 1,318 & 0,759 & 6,90 & 3,98 & 2,85 \\
\hline Le, Sumiava, Lana tane & LAK 04 & 10.9 .2010 & 1,427 & 0,701 & 6,37 & 3,81 & 3,21 \\
\hline & LAK 05 & 10.9 .2010 & 1,429 & 0,700 & 6,36 & 3,13 & 2,81 \\
\hline & LAK 06 & 10.9 .2010 & 1,282 & 0,780 & 7,09 & 3,04 & 3,45 \\
\hline & PRIL 01+P & 12.7 .2010 & 1,261 & 0,793 & 7,21 & 2,86 & 2,45 \\
\hline & PRIL 02 & 12.7 .2010 & 1,262 & 0,792 & 7,20 & 3,35 & 2,05 \\
\hline & PRIL 03 & 12.7 .2010 & 1,670 & 0,599 & 5,44 & 2,90 & 3,22 \\
\hline & PRIL 04 & 12.7 .2010 & 1,254 & 0,797 & 7,25 & 3,27 & 2,68 \\
\hline & PRIL 05 & 12.7 .2010 & 1,250 & 0,800 & 7,27 & 3,08 & 2,80 \\
\hline & PRIL 06 & 12.7 .2010 & 1,459 & 0,685 & 6,23 & 3,44 & 2,46 \\
\hline & PRIL 07 & 12.7 .2010 & 1,619 & 0,618 & 5,61 & 3,32 & 3,91 \\
\hline & PRIL 1 & 18.10.2011 & 1,251 & 0,799 & 7,27 & 2,69 & 2,60 \\
\hline & & 24.10.2011 & 1,271 & 0,787 & 7,15 & 2,66 & 2,03 \\
\hline & & 1.11 .2011 & 1,255 & 0,797 & 7,24 & 2,97 & 2,47 \\
\hline & PRIL 2 & 18.10.2011 & 1,643 & 0,609 & 5,53 & 2,15 & 2,42 \\
\hline & & 24.10.2011 & 1,650 & 0,606 & 5,51 & 2,75 & 2,04 \\
\hline CZ, Šumava, Nové Hutě & & 1.11 .2011 & 1,629 & 0,614 & 5,58 & 3,09 & 2,76 \\
\hline & PRIL 3 & 18.10 .2011 & 1,647 & 0,607 & 5,52 & 2,90 & 2,59 \\
\hline & & 24.10.2011 & 1,649 & 0,606 & 5,51 & 2,96 & 2,03 \\
\hline & & 31.10 .2011 & 1,638 & 0,611 & 5,55 & 2,71 & 1,93 \\
\hline & PRIL 4 & 18.10 .2011 & 1,643 & 0,609 & 5,53 & 2,61 & 2,00 \\
\hline & & 31.10 .2011 & 1,638 & 0,611 & 5,55 & 2,85 & 2,52 \\
\hline
\end{tabular}




\begin{tabular}{|c|c|c|c|c|c|c|c|}
\hline & \multirow{3}{*}{ PRIL 5} & 2.11 .2011 & 1,622 & 0,617 & 5,60 & 2,70 & 2,59 \\
\hline & & 1.11 .2011 & 1,230 & 0,813 & 7,39 & 2,10 & 2,77 \\
\hline & & 2.11 .2011 & 1,259 & 0,794 & 7,22 & 2,71 & 2,21 \\
\hline & \multirow{4}{*}{ PRIL 6} & 3.11 .2011 & 1,240 & 0,806 & 7,33 & 2,32 & 2,17 \\
\hline & & 1.11 .2011 & 1,608 & 0,622 & 5,65 & 3,06 & 2,35 \\
\hline & & 2.11.2011 & 1,680 & 0,595 & 5,41 & 2,41 & 2,21 \\
\hline & & 3.11 .2011 & 1,664 & 0,601 & 5,46 & 2,72 & 1,88 \\
\hline \multirow{6}{*}{ CZ, Šumava, Ostrá hora } & OST 01+P & 9.7 .2010 & 1,713 & 0,584 & 5,31 & 2,54 & 1,82 \\
\hline & OST 02 & 9.7 .2010 & 1,721 & 0,581 & 5,28 & 2,73 & 1,91 \\
\hline & OST 03 & 9.7 .2010 & 1,722 & 0,581 & 5,28 & 2,54 & 2,12 \\
\hline & OST 04 & 9.7 .2010 & 1,710 & 0,585 & 5,32 & 3,02 & 1,83 \\
\hline & OST 05 & 9.7 .2010 & 1,716 & 0,583 & 5,30 & 3,08 & 1,94 \\
\hline & OST 06 & 9.7.2010 & 1,747 & 0,572 & 5,20 & 2,50 & 1,97 \\
\hline \multirow{10}{*}{ CZ, Šumava, VItava's springs } & VLT 01+P & 10.9.2010 & 1,265 & 0,791 & 7,19 & 2,53 & 2,66 \\
\hline & VLT 02 & 10.9.2010 & 1,271 & 0,787 & 7,15 & 3,32 & 3,30 \\
\hline & VLT 03 & 10.9 .2010 & 1,234 & 0,810 & 7,37 & 3,04 & 3,20 \\
\hline & VLT 04 & 10.9.2010 & 1,417 & 0,706 & 6,41 & 2,85 & 3,13 \\
\hline & VLT 05 & 10.9 .2010 & 1,263 & 0,792 & 7,20 & 3,69 & 3,11 \\
\hline & VLT 06 & 10.9.2010 & 1,441 & 0,694 & 6,31 & 3,69 & 3,45 \\
\hline & VLT 07 & 10.9 .2010 & 1,539 & 0,650 & 5,91 & 4,87 & 3,48 \\
\hline & VLT 07 & 4.11 .2010 & 1,583 & 0,632 & 5,74 & 3,86 & 2,40 \\
\hline & VLT 07_2 & 3.3.2011 & 1,581 & 0,633 & 5,75 & 4,62 & 2,11 \\
\hline & VLT 08 & 10.9 .2010 & 1,441 & 0,694 & 6,31 & 4,03 & 2,91 \\
\hline \multirow{5}{*}{ CZ, Šumava, Příslop } & PRI 01+P & 9.7 .2010 & 1,646 & 0,608 & 5,52 & 2,57 & 1,81 \\
\hline & PRI 02 & 9.7 .2010 & 1,625 & 0,615 & 5,59 & 2,85 & 2,09 \\
\hline & PRI 03 & 9.7 .2010 & 1,634 & 0,612 & 5,56 & 3,16 & 2,10 \\
\hline & PRI 04 (1.pq & 9.7 .2010 & 1,661 & 0,602 & 5,47 & 2,89 & 1,90 \\
\hline & PRI 05 & 9.7.2010 & 1,665 & 0,601 & 5,46 & 2,79 & 1,98 \\
\hline \multirow{7}{*}{ CZ, Šumava, Srní } & SRN 01+P & 9.9 .2010 & 1,495 & 0,669 & 6,08 & 4,18 & 3,31 \\
\hline & SRN 01 & 4.11 .2010 & 1,526 & 0,655 & 5,96 & 3,56 & 2,30 \\
\hline & SRN 01 & 3.3.2011 & 1,521 & 0,657 & 5,98 & 2,63 & 1,39 \\
\hline & SRN 02 & 9.9 .2010 & 1,495 & 0,669 & 6,08 & 4,13 & 3,66 \\
\hline & SRN & 24.10 .2011 & 1,491 & 0,671 & 6,10 & 2,67 & 2,04 \\
\hline & & 26.10 .2011 & 1,470 & 0,680 & 6,18 & 2,01 & 2,51 \\
\hline & & 31.10 .2011 & 1,495 & 0,669 & 6,08 & 2,94 & 2,17 \\
\hline \multirow{2}{*}{ CZ, Šumava, Stachy } & STA 1 & 8.9 .2011 & 1,420 & 0,704 & 6,40 & 2,12 & 1,58 \\
\hline & STA 2 & 8.9.2011 & 1,401 & 0,714 & 6,49 & 2,86 & 1,44 \\
\hline & $S \check{1} 1+P$ & 22.10 .2009 & 1,282 & 0,780 & 7,09 & 2,75 & 2,31 \\
\hline & $S \check{2}+P$ & 22.10 .2009 & 1,426 & 0,701 & 6,37 & 2,75 & 1,91 \\
\hline & $5 \check{3} 3+P$ & 22.10 .2009 & 1,261 & 0,793 & 7,21 & 2,31 & 2,18 \\
\hline & $5 \check{4}+P$ & 22.10 .2009 & 1,246 & 0,803 & 7,30 & 2,49 & 2,21 \\
\hline & Š5+P & 22.10 .2009 & 1,265 & 0,791 & 7,19 & 2,93 & 2,47 \\
\hline & $5 \check{5} 6+P$ & 22.10 .2009 & 1,243 & 0,805 & 7,31 & 2,65 & 2,56 \\
\hline & Š7+P & 22.10 .2009 & 1,434 & 0,697 & 6,34 & 3,10 & 1,82 \\
\hline & Š8+P & 22.10 .2009 & 1,317 & 0,759 & 6,90 & 3,11 & 2,35 \\
\hline & Šg+P & 22.10 .2009 & 1,240 & 0,806 & 7,33 & 2,66 & 2,68 \\
\hline & Š10+P & 22.10 .2009 & 1,249 & 0,801 & 7,28 & 2,60 & 2,09 \\
\hline & Š11+P & 22.10 .2009 & 1,249 & 0,801 & 7,28 & 2,37 & 2,09 \\
\hline & $2 S ̌ 1+P$ & 22.10 .2009 & 1,792 & 0,558 & 5,07 & 2,38 & 2,12 \\
\hline & $2 \check{S} 2+P$ & 22.10 .2009 & 1,277 & 0,783 & 7,12 & 2,98 & 2,14 \\
\hline
\end{tabular}




\begin{tabular}{|c|c|c|c|c|c|c|c|}
\hline \multirow[t]{14}{*}{ |CZ, Šumava, Špičák hill } & $2 \check{2} 3+P$ & 22.10 .2009 & 1,774 & 0,564 & 5,12 & 2,83 & 2,08 \\
\hline & $25 \check{4}+\mathrm{P}$ & 22.10 .2009 & 1,248 & 0,801 & 7,28 & 2,42 & 1,76 \\
\hline & $25 \check{5}+\mathrm{P}$ & 22.10 .2009 & 1,274 & 0,785 & 7,14 & 2,91 & 2,53 \\
\hline & $25 \check{6} 6+P$ & 22.10 .2009 & 1,259 & 0,794 & 7,22 & 2,94 & 2,10 \\
\hline & $25 \check{7}+P$ & 22.10 .2009 & 1,282 & 0,780 & 7,09 & 2,50 & 2,15 \\
\hline & $25 \check{8}+P$ & 22.10 .2009 & 1,438 & 0,695 & 6,32 & 2,51 & 2,17 \\
\hline & $25 \check{9}+P$ & 22.10 .2009 & 1,271 & 0,787 & 7,15 & 2,56 & 2,59 \\
\hline & $2 S ̌ 10+P$ & 22.10 .2009 & 1,270 & 0,787 & 7,16 & 2,87 & 2,24 \\
\hline & $2 s ̌ 11+P$ & 22.10 .2009 & 1,244 & 0,804 & 7,31 & 2,62 & 2,17 \\
\hline & $25 ̌ 11+P$ & 23.10 .2009 & 1,242 & 0,805 & 7,32 & 2,51 & 2,14 \\
\hline & $2 \breve{12+P}$ & 23.10 .2009 & 1,226 & 0,816 & 7,41 & 2,85 & 2,21 \\
\hline & $2 S ̌ 13+P$ & 23.10 .2009 & 1,237 & 0,808 & 7,35 & 2,98 & 1,86 \\
\hline & $25 ั 14+P$ & 23.10 .2009 & 1,219 & 0,820 & 7,46 & 2,85 & 2,60 \\
\hline & $2 S ̌ 15+P$ & 23.10.2009 & 1,242 & 0,805 & 7,32 & 2,51 & 2,14 \\
\hline \multirow{10}{*}{$\begin{array}{l}\text { CZ, Šumava, Včelná pod } \\
\text { Boubínem }\end{array}$} & VCE 01+P & 9.7 .2010 & 1,616 & 0,619 & 5,63 & 2,77 & 2,24 \\
\hline & VCE 02 & 9.7 .2010 & 1,642 & 0,609 & 5,54 & 2,66 & 1,92 \\
\hline & VCE 03 & 9.7 .2010 & 1,421 & 0,704 & 6,40 & 2,78 & 2,16 \\
\hline & VCE 04 & 12.7 .2010 & 1,432 & 0,698 & 6,35 & 1,84 & 2,81 \\
\hline & VCE 1 & 18.10.2011 & 1,551 & 0,645 & 5,86 & 2,80 & 2,39 \\
\hline & & 31.10 .2011 & 1,624 & 0,616 & 5,60 & 3,02 & 2,93 \\
\hline & & 1.11.2011 & 1,604 & 0,623 & 5,67 & 2,60 & 2,22 \\
\hline & VCE 2 & 24.10 .2011 & 1,466 & 0,682 & 6,20 & 2,40 & 2,02 \\
\hline & & 31.10 .2011 & 1,454 & 0,688 & 6,25 & 2,55 & 1,60 \\
\hline & & 1.11.2011 & 1,453 & 0,688 & 6,26 & 2,56 & 2,18 \\
\hline \multirow{9}{*}{$\begin{array}{l}\text { CZ, Šumava, Vysočina - } \\
\text { Čerínek }\end{array}$} & CER 01+P & 10.9 .2010 & 1,695 & 0,590 & 5,36 & 3,14 & 2,11 \\
\hline & CER 02 & 10.9 .2010 & 1,686 & 0,593 & 5,39 & 3,71 & 2,60 \\
\hline & CER 03 & 10.9 .2010 & 1,638 & 0,611 & 5,55 & 4,07 & 2,34 \\
\hline & CER 04 & 10.9.2010 & 1,748 & 0,572 & 5,20 & 4,29 & 2,07 \\
\hline & CER 04 & 4.11 .2010 & 1,760 & 0,568 & 5,16 & 2,92 & 1,78 \\
\hline & CER 04 & 3.3.2011 & 1,685 & 0,593 & 5,39 & 3,87 & 1,81 \\
\hline & CER 05 & 3.3.2011 & 1,312 & 0,762 & 6,93 & 3,97 & 1,69 \\
\hline & CER 05 & 4.11 .2010 & 1,437 & 0,696 & 6,33 & 2,98 & 2,42 \\
\hline & CER 05 & 3.3.2011 & 1,312 & 0,762 & 6,93 & 3,97 & 1,69 \\
\hline \multirow{9}{*}{ CZ, Šumava, Zadov } & $\mathrm{Z1+P}$ & 23.10 .2009 & 1,224 & 0,817 & 7,43 & 2,48 & 2,24 \\
\hline & $\mathrm{Z2}+\mathrm{P}$ & 23.10 .2009 & 1,243 & 0,805 & 7,31 & 1,87 & 2,79 \\
\hline & $z 3+p$ & 23.10.2009 & 1,212 & 0,825 & 7,50 & 2,30 & 2,18 \\
\hline & $Z 4+P$ & 23.10.2009 & 1,244 & 0,804 & 7,31 & 2,56 & 1,98 \\
\hline & $25+P$ & 23.10 .2009 & 1,230 & 0,813 & 7,39 & 2,74 & 1,89 \\
\hline & $26+P$ & 23.10 .2009 & 1,222 & 0,818 & 7,44 & 2,47 & 2,04 \\
\hline & ZAD 1 & 24.10 .2011 & 1,280 & 0,781 & 7,10 & 2,76 & 2,14 \\
\hline & & 31.10 .2011 & 1,253 & 0,798 & 7,25 & 2,40 & 2,05 \\
\hline & & 2.11 .2011 & 1,255 & 0,797 & 7,24 & 2,96 & 2,87 \\
\hline \multirow{8}{*}{ CZ, Šumava, Zadov } & CPL 01+P & 12.7.2010 & 1,269 & 0,788 & 7,16 & 1,87 & 2,04 \\
\hline & CPL 02 & 12.7 .2010 & 1,262 & 0,792 & 7,20 & 2,78 & 2,67 \\
\hline & CPL 03 & 12.7 .2010 & 1,270 & 0,787 & 7,16 & 2,18 & 2,34 \\
\hline & CPL 04 & 12.7 .2010 & 1,276 & 0,784 & 7,12 & 3,43 & 2,33 \\
\hline & CPL 05 & 12.7 .2010 & 1,261 & 0,793 & 7,21 & 3,12 & 2,27 \\
\hline & CPL 06 & 12.7 .2010 & 1,260 & 0,794 & 7,21 & 2,57 & 2,11 \\
\hline & ZMU 01+P & 12.7 .2010 & 1,254 & 0,797 & 7,25 & 3,20 & 2,47 \\
\hline & ZMU 02 & 12.7.2010 & 1,264 & 0,791 & 7,19 & 3,29 & 2,11 \\
\hline
\end{tabular}




\begin{tabular}{|c|c|c|c|c|c|c|c|}
\hline \multirow{18}{*}{ CZ, Šumava, Žlíbský hill } & ZLI 01 & 40330 & 1,464 & 0,683 & 6,21 & 4,19 & 2,74 \\
\hline & ZLI 02/1 & 40330 & 1,745 & 0,573 & 5,21 & 3,28 & 2,83 \\
\hline & ZLI 02/2 & 40330 & 1,759 & 0,569 & 5,17 & 3,05 & 2,42 \\
\hline & ZLI 03 & 40330 & 1,708 & 0,585 & 5,32 & 3,41 & 1,67 \\
\hline & ZLI 04 & 40330 & 1,765 & 0,567 & 5,15 & 3,03 & 2,70 \\
\hline & ZLI 05 & 1.6.2010 & 1,759 & 0,569 & 5,17 & 2,50 & 1,78 \\
\hline & ZLI 06 & 7.6.2010 & 1,468 & 0,681 & 6,19 & 4,28 & 3,74 \\
\hline & ZLI 07 & 1.6.2010 & 1,762 & 0,568 & 5,16 & 3,68 & 2,68 \\
\hline & ZLI 08 & 1.6.2010 & 1,274 & 0,785 & 7,14 & 2,92 & 2,45 \\
\hline & ZLI 09/1 & 1.6.2010 & 1,753 & 0,570 & 5,19 & 3,26 & 2,91 \\
\hline & ZLI 09/2 & 1.6.2010 & 1,425 & 0,702 & 6,38 & 2,91 & 2,60 \\
\hline & ZLI 10 & 1.6.2010 & 1,748 & 0,572 & 5,20 & 3,20 & 2,89 \\
\hline & ZLI 11 & 7.6.2010 & 1,242 & 0,805 & 7,32 & 5,62 & 3,78 \\
\hline & ZLI 12 & 7.6.2010 & 1,194 & 0,838 & 7,61 & 3,84 & 4,31 \\
\hline & ZLI 13 & 7.6.2010 & 1,510 & 0,662 & 6,02 & 4,63 & 3,26 \\
\hline & ZLI 14 & 7.6.2010 & 1,647 & 0,607 & 5,52 & 5,11 & 4,08 \\
\hline & ZLI 15/1 & 7.6.2010 & 1,744 & 0,573 & 5,21 & 4,68 & 3,28 \\
\hline & ZLI 15/2 & 7.6.2010 & 1,772 & 0,564 & 5,13 & 5,19 & 4,30 \\
\hline \multirow{32}{*}{ CZ, Terezín, Červenka forest } & KB1+P & 10.12.2009 & 1,866 & 0,536 & 4,87 & 2,71 & 1,85 \\
\hline & KB2+P & 10.12 .2009 & 1,669 & 0,599 & 5,45 & 2,59 & 1,90 \\
\hline & KB3+P & 10.12 .2009 & 1,659 & 0,603 & 5,48 & 2,33 & 1,85 \\
\hline & KB4+P & 10.12 .2009 & 1,804 & 0,554 & 5,04 & 3,05 & 2,37 \\
\hline & KB5+P & 10.12 .2009 & 1,666 & 0,600 & 5,46 & 2,95 & 2,33 \\
\hline & KB6+P & 10.12 .2009 & 1,862 & 0,537 & 4,88 & 3,40 & 2,48 \\
\hline & KB7+P & 10.12 .2009 & 1,679 & 0,596 & 5,41 & 3,15 & 1,89 \\
\hline & KB8+P & 10.12 .2009 & 1,823 & 0,549 & 4,99 & 3,06 & 2,51 \\
\hline & KB9+P & 10.12 .2009 & 1,660 & 0,602 & 5,48 & 2,59 & 1,60 \\
\hline & KB10+P & 10.12 .2009 & 1,868 & 0,535 & 4,87 & 2,57 & 1,60 \\
\hline & KB11+P & 10.12 .2009 & 1,883 & 0,531 & 4,83 & 3,33 & 1,92 \\
\hline & KB12+P & 10.12 .2009 & 1,666 & 0,600 & 5,46 & 2,35 & 1,29 \\
\hline & KB13+P & 10.12 .2009 & 1,910 & 0,524 & 4,76 & 3,97 & 2,90 \\
\hline & KB14+P & 15.12 .2009 & 1,838 & 0,544 & 4,95 & 2,58 & 2,62 \\
\hline & KB15+P & 15.12 .2009 & 1,848 & 0,541 & 4,92 & 2,53 & 1,89 \\
\hline & KB16+P & 15.12 .2009 & 1,652 & 0,605 & 5,50 & 2,21 & 1,38 \\
\hline & KB17+P & 15.12 .2009 & 1,821 & 0,549 & 4,99 & 2,06 & 2,44 \\
\hline & KB18+P & 15.12 .2009 & 1,634 & 0,612 & 5,56 & 1,93 & 1,81 \\
\hline & KB19+P & 15.12 .2009 & 1,663 & 0,601 & 5,47 & 2,16 & 1,69 \\
\hline & KB20+P & 15.12 .2009 & 1,644 & 0,608 & 5,53 & 2,53 & 1,71 \\
\hline & KB21+P & 15.12 .2009 & 1,591 & 0,629 & 5,71 & 2,74 & 1,81 \\
\hline & KB22+P & 15.12 .2009 & 1,657 & 0,604 & 5,49 & 2,45 & 1,86 \\
\hline & KB23+P & 15.12 .2009 & 1,837 & 0,544 & 4,95 & 2,43 & 1,95 \\
\hline & KB24+P & 15.12 .2009 & 1,833 & 0,546 & 4,96 & 1,95 & 1,09 \\
\hline & KB25+P & 14.12 .2009 & 1,635 & 0,612 & 5,56 & 2,17 & 2,97 \\
\hline & KB26+P & 15.12 .2009 & 1,828 & 0,547 & 4,97 & 2,28 & 1,37 \\
\hline & KB27+P & 15.12 .2009 & 1,666 & 0,600 & 5,46 & 2,32 & 1,73 \\
\hline & KB28+P & 15.12 .2009 & 1,809 & 0,553 & 5,02 & 3,38 & 2,17 \\
\hline & KB29+P & 14.12 .2009 & 1,790 & 0,559 & 5,08 & 2,08 & 2,84 \\
\hline & KB30+P & 14.12 .2009 & 1,791 & 0,558 & 5,08 & 3,37 & 2,74 \\
\hline & $\mathrm{T} 1+\mathrm{P}$ & 14.12 .2009 & 1,636 & 0,611 & 5,56 & 2,93 & 2,20 \\
\hline & $T 2+P$ & 14.12 .2009 & 1,818 & 0,550 & 5,00 & 3,66 & 2,56 \\
\hline
\end{tabular}




\begin{tabular}{|c|c|c|c|c|c|c|c|}
\hline \multirow{8}{*}{ CZ, Terezín, Červenka forest } & $\mathrm{T} 3+\mathrm{P}$ & 14.12 .2009 & 1,810 & 0,552 & 5,02 & 3,36 & 2,28 \\
\hline & $\mathrm{T} 4+\mathrm{P}$ & 14.12.2009 & 1,723 & 0,580 & 5,28 & 3,29 & 1,96 \\
\hline & $\mathrm{T} 5+\mathrm{P}$ & 14.12.2009 & 1,586 & 0,631 & 5,73 & 2,10 & 2,45 \\
\hline & $\mathrm{T} 6+\mathrm{P}$ & 14.12 .2009 & 1,682 & 0,595 & 5,40 & 2,86 & 2,04 \\
\hline & $\mathrm{T} 7+\mathrm{P}$ & 15.12 .2009 & 1,776 & 0,563 & 5,12 & 2,95 & 1,67 \\
\hline & T8+P & 14.12.2009 & 1,786 & 0,560 & 5,09 & 2,77 & 2,32 \\
\hline & T9+P & 14.12.2009 & 1,723 & 0,580 & 5,28 & 2,63 & 1,72 \\
\hline & $\mathrm{T} 10+\mathrm{P}$ & 14.12.2009 & 1,813 & 0,552 & 5,01 & 2,83 & 2,10 \\
\hline \multirow{7}{*}{ CZ, Větrný Jeníkov } & VJ1+P & 26.10 .2009 & 1,638 & 0,611 & 5,55 & 2,62 & 1,97 \\
\hline & VJ1 & 10.12 .2009 & 1,643 & 0,609 & 5,53 & 3,33 & 2,03 \\
\hline & $V J 2+P$ & 26.10 .2009 & 1,659 & 0,603 & 5,48 & 2,96 & 2,29 \\
\hline & VJ3+P & 26.10 .2009 & 1,700 & 0,588 & 5,35 & 2,97 & 2,72 \\
\hline & VJ3 & 10.12 .2009 & 1,674 & 0,597 & 5,43 & 2,78 & 1,78 \\
\hline & VJ4+P & 26.10 .2009 & 1,657 & 0,604 & 5,49 & 2,53 & 2,27 \\
\hline & VJ5+P & 26.10 .2009 & 1,654 & 0,605 & 5,50 & 2,73 & 2,14 \\
\hline A, Alps, Oberatuern & RAK & 18.10.2011 & 1,206 & 0,829 & 7,54 & 2,85 & 2,37 \\
\hline \multirow{34}{*}{ A, Hochficht } & $\mathrm{HOCH} 1$ & 18.10 .2007 & 1,219 & 0,820 & 7,46 & 2,30 & \\
\hline & $\mathrm{HOCH} 2$ & 18.10 .2007 & 1,236 & 0,809 & 7,35 & 2,76 & \\
\hline & $\mathrm{HOCH} 3$ & 18.10 .2007 & 1,241 & 0,806 & 7,32 & 2,85 & \\
\hline & $\mathrm{HOCH} 4$ & 19.10 .2007 & 1,252 & 0,799 & 7,26 & 2,79 & \\
\hline & $\mathrm{HOCH} 5$ & 19.10.2007 & 1,255 & 0,797 & 7,24 & 2,28 & \\
\hline & $\mathrm{HOCH} 6$ & 19.10 .2007 & 1,256 & 0,796 & 7,24 & 2,99 & \\
\hline & $\mathrm{HOCH} 7$ & 19.10 .2007 & 1,258 & 0,795 & 7,23 & 3,00 & \\
\hline & $\mathrm{HOCH} 8$ & 19.10.2007 & 1,261 & 0,793 & 7,21 & 2,40 & \\
\hline & $\mathrm{HOCH} 9$ & 19.10.2007 & 1,262 & 0,792 & 7,20 & 4,23 & \\
\hline & $\mathrm{HOCH} 10$ & 19.10.2007 & 1,264 & 0,791 & 7,19 & 2,86 & \\
\hline & $\mathrm{HOCH} 11$ & 19.10.2007 & 1,264 & 0,791 & 7,19 & 3,15 & \\
\hline & $\mathrm{HOCH} 12$ & 19.10.2007 & 1,265 & 0,791 & 7,19 & 2,23 & \\
\hline & $\mathrm{HOCH} 13$ & 19.10.2007 & 1,266 & 0,790 & 7,18 & 3,37 & \\
\hline & $\mathrm{HOCH} 14$ & 19.10.2007 & 1,267 & 0,789 & 7,17 & 4,08 & \\
\hline & $\mathrm{HOCH} 15$ & 19.10.2007 & 1,267 & 0,789 & 7,17 & 1,22 & \\
\hline & $\mathrm{HOCH} 16$ & 19.10 .2007 & 1,268 & 0,789 & 7,17 & 4,37 & \\
\hline & $\mathrm{HOCH} 17$ & 19.10 .2007 & 1,268 & 0,789 & 7,17 & 3,20 & \\
\hline & $\mathrm{HOCH} 18$ & 19.10.2007 & 1,268 & 0,789 & 7,17 & 3,97 & \\
\hline & $\mathrm{HOCH} 19$ & 19.10.2007 & 1,271 & 0,787 & 7,15 & 2,81 & \\
\hline & $\mathrm{HOCH} 20$ & 19.10.2007 & 1,272 & 0,786 & 7,15 & 2,74 & \\
\hline & $\mathrm{HOCH} 21$ & 19.10 .2007 & 1,275 & 0,784 & 7,13 & 2,22 & \\
\hline & $\mathrm{HOCH} 22$ & 19.10.2007 & 1,277 & 0,783 & 7,12 & 4,32 & \\
\hline & $\mathrm{HOCH} 23$ & 19.10.2007 & 1,279 & 0,782 & 7,11 & 2,46 & \\
\hline & $\mathrm{HOCH} 24$ & 19.10.2007 & 1,285 & 0,778 & 7,07 & 2,61 & \\
\hline & $\mathrm{HOCH} 25$ & 19.10.2007 & 1,287 & 0,777 & 7,06 & 2,98 & \\
\hline & $\mathrm{HOCH} 26$ & 19.10.2007 & 1,292 & 0,774 & 7,04 & 2,85 & \\
\hline & $\mathrm{HOCH} 27$ & 19.10 .2007 & 1,292 & 0,774 & 7,04 & 4,22 & \\
\hline & $\mathrm{HOCH} 28$ & 19.10.2007 & 1,292 & 0,774 & 7,04 & 4,09 & \\
\hline & $\mathrm{HOCH} 29$ & 19.10.2007 & 1,297 & 0,771 & 7,01 & 4,12 & \\
\hline & $\mathrm{HOCH} 30$ & 19.10.2007 & 1,298 & 0,770 & 7,00 & 2,88 & \\
\hline & $\mathrm{HOCH} 31$ & 24.10 .2007 & 1,303 & 0,767 & 6,98 & 2,57 & \\
\hline & HOCH32 & 24.10 .2007 & 1,308 & 0,765 & 6,95 & 2,78 & \\
\hline & $\mathrm{HOCH} 48$ & 30.10 .2007 & 1,616 & 0,619 & 5,63 & 2,67 & \\
\hline & HOCH49 & 31.10 .2007 & 1,619 & 0,618 & 5,61 & 2,45 & \\
\hline
\end{tabular}




\begin{tabular}{|c|c|c|c|c|c|c|c|}
\hline & $\mathrm{HOCH} 50$ & 31.10 .2007 & 1,630 & 0,613 & 5,58 & 2,35 & \\
\hline & HOCH51 & 12.11 .2007 & 1,728 & 0,579 & 5,26 & 3,31 & \\
\hline & HOCH52 & 13.11.2007 & 1,729 & 0,578 & 5,26 & 3,53 & \\
\hline & $\mathrm{HOCH} 53$ & 13.11 .2007 & 1,742 & 0,574 & 5,22 & 3,50 & \\
\hline & $\mathrm{HOCH} 54$ & 13.11 .2007 & 1,748 & 0,572 & 5,20 & 3,12 & \\
\hline & HOCH55 & 13.11.2007 & 1,764 & 0,567 & 5,15 & 2,60 & \\
\hline & HOCH56 & 14.11.2007 & 1,766 & 0,566 & 5,15 & 2,23 & \\
\hline & HOCH57 & 14.11.2007 & 1,769 & 0,565 & 5,14 & 3,08 & \\
\hline & $\mathrm{HOCH} 58$ & 14.11 .2007 & 1,782 & 0,561 & 5,10 & 4,12 & \\
\hline & HOCH59 & 14.11.2007 & 1,796 & 0,557 & 5,06 & 2,82 & \\
\hline & $\mathrm{HOCH} 60$ & 14.11.2007 & 1,817 & 0,550 & 5,00 & 3,11 & \\
\hline & HOCH61 & 14.11.2007 & 1,825 & 0,548 & 4,98 & 3,64 & \\
\hline & HOCH62 & 14.11 .2007 & 1,828 & 0,547 & 4,97 & 4,16 & \\
\hline & $\mathrm{HOCH} 63$ & 14.11 .2007 & 1,833 & 0,546 & 4,96 & 3,83 & \\
\hline & $\mathrm{HOCH} 64$ & 14.11.2007 & 1,841 & 0,543 & 4,94 & 3,65 & \\
\hline & HOCH65 & 14.11.2007 & 1,848 & 0,541 & 4,92 & 3,63 & \\
\hline & $\mathrm{HOCH} 66$ & 14.11.2007 & 1,848 & 0,541 & 4,92 & 2,46 & \\
\hline & $\mathrm{HOCH} 67$ & 14.11.2007 & 1,854 & 0,539 & 4,90 & 3,01 & \\
\hline & $\mathrm{HOCH} 68$ & 14.11 .2007 & 1,863 & 0,537 & 4,88 & 2,90 & \\
\hline & HOCH69 & 14.11.2007 & 1,872 & 0,534 & 4,86 & 3,61 & \\
\hline & $\mathrm{HOCH} 70$ & 14.11.2007 & 1,879 & 0,532 & 4,84 & 4,67 & \\
\hline & HOCH71 & 14.11.2007 & 1,883 & 0,531 & 4,83 & 4,86 & \\
\hline & $\mathrm{HOCH} 72$ & 14.11 .2007 & 1,887 & 0,530 & 4,82 & 2,15 & \\
\hline & 12-09-A-1 & 7.9.2009 & 1,281 & 0,781 & 7,10 & 2,59 & 2,62 \\
\hline $\begin{array}{l}\text { A, Karnten, Uberkoibnitz, } \\
\text { Reisseck-gruppe }\end{array}$ & $12-09-A-2$ & 7.9 .2009 & 1,271 & 0,787 & 7,15 & 2,57 & 2,57 \\
\hline & 12-09-A-3 & 7.9.2009 & 1,263 & 0,792 & 7,20 & 2,32 & 2,61 \\
\hline F, Störsvik & FIN 8-1 & 8.9 .2011 & 1,673 & 0,598 & 5,43 & 2,26 & 1,77 \\
\hline & FIN 8-2 & 8.9 .2011 & 1,671 & 0,598 & 5,44 & 1,91 & 1,64 \\
\hline & FIN 5-1 & 8.9 .2011 & 1,641 & 0,609 & 5,54 & 2,01 & 1,12 \\
\hline F, Uusimaa, Nurmijärvi & FIN 5-2 & 5.9 .2011 & 1,655 & 0,604 & 5,49 & 2,64 & 2,52 \\
\hline & FIN 5-3 & 5.9.2011 & 1,670 & 0,599 & 5,44 & 2,59 & 2,38 \\
\hline F, Uusimaa, Nurmijärvi, & FIN 3-1 & 8.9 .2011 & 1,664 & 0,601 & 5,46 & 2,47 & 1,46 \\
\hline Kiljava & FIN 3-2 & 8.9 .2011 & 1,683 & 0,594 & 5,40 & 2,14 & 1,57 \\
\hline F, Uusimaa, Nurmijärvi, & FIN 4-1 & 6.9 .2011 & 1,678 & 0,596 & 5,42 & 2,59 & 2,94 \\
\hline Kiljava & FIN 4-2 & 6.9 .2011 & 1,743 & 0,574 & 5,22 & 3,10 & 2,85 \\
\hline IF Uusimaa Tammisaari & FIN 1-1+P & 6.9 .2011 & 1,747 & 0,572 & 5,20 & 2,88 & 2,63 \\
\hline & FIN 1-2 & 6.9 .2011 & 1,771 & 0,565 & 5,13 & 2,95 & 2,75 \\
\hline F, Varsinais-Suomi & FIN 6-1 & 8.9 .2011 & 1,663 & 0,601 & 5,47 & 2,82 & 1,46 \\
\hline & FIN 6-2 & 6.9 .2011 & 1,770 & 0,565 & 5,14 & 2,93 & 2,75 \\
\hline F Varsinais-Suomi & FIN 7-1 & 6.9 .2011 & 1,684 & 0,594 & 5,40 & 2,98 & 2,98 \\
\hline 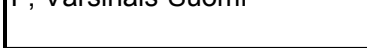 & FIN 7-2 & 6.9 .2011 & 1,712 & 0,584 & 5,31 & 2,68 & 2,47 \\
\hline $\begin{array}{l}\text { F, Varsinais-Suomi, Kiikala, } \\
\text { Tarvakas }\end{array}$ & FIN 2 & 8.9 .2011 & 1,696 & 0,590 & 5,36 & 2,29 & 1,64 \\
\hline & SK 1-1 & 5.9 .2011 & 1,272 & 0,786 & 7,15 & 3,40 & 2,42 \\
\hline GB, Scotland & SK 1-2 & 8.9 .2011 & 1,252 & 0,799 & 7,26 & 2,07 & 1,58 \\
\hline & SK 1-3 & 8.9 .2011 & 1,249 & 0,801 & 7,28 & 2,33 & 1,44 \\
\hline GB Scotland & SK 2-1 & 5.9.2011 & 1,266 & 0,790 & 7,18 & 3,57 & 2,62 \\
\hline & SK 2-2 & 5.9.2011 & 1,271 & 0,787 & 7,15 & 3,73 & 3,01 \\
\hline & SK 3-C & 5.9.2011 & 1,279 & 0,782 & 7,11 & 2,40 & 2,24 \\
\hline & SK 3a - 1 & 5.9 .2011 & 1,240 & 0,806 & 7,33 & 3,01 & 3,18 \\
\hline
\end{tabular}




\begin{tabular}{|c|c|c|c|c|c|c|c|}
\hline \multirow{5}{*}{ GB, Scotland } & SK 3a - 2 & 5.9.2011 & 1,241 & 0,806 & 7,32 & 2,88 & 2,90 \\
\hline & SK $3 a-3$ & 5.9 .2011 & 1,263 & 0,792 & 7,20 & 2,27 & 2,33 \\
\hline & SK 3b -1 & 5.9 .2011 & 1,276 & 0,784 & 7,12 & 2,25 & 2,77 \\
\hline & SK $3 b-2$ & 5.9 .2011 & 1,293 & 0,773 & 7,03 & 1,89 & 2,38 \\
\hline & SK 3d & 5.9.2011 & 1,288 & 0,776 & 7,06 & 2,96 & 2,88 \\
\hline \multirow{4}{*}{ GB, Scotland } & SK 4-2_2 & 8.9.2011 & 1,248 & 0,801 & 7,28 & 2,82 & 1,45 \\
\hline & SK 4-3 & 6.9 .2011 & 1,278 & 0,782 & 7,11 & 2,28 & 2,36 \\
\hline & SK 4-4 & 6.9 .2011 & 1,291 & 0,775 & 7,04 & 3,03 & 2,58 \\
\hline & SK 4-5 & 8.9.2011 & 1,261 & 0,793 & 7,21 & 2,26 & 1,43 \\
\hline GB, Scotland, Knockhan Crag & VB & 24.10 .2011 & 1,256 & 0,796 & 7,24 & 2,19 & 2,07 \\
\hline $\mathrm{N}$, Alnesvatnet & SC3 & 7.9.2009 & 1,280 & 0,781 & 7,10 & 2,51 & 2,33 \\
\hline N, Dalsvelen & SC5 & 7.9.2009 & 1,675 & 0,597 & 5,43 & 2,64 & 2,61 \\
\hline \multirow{3}{*}{ N, Järvsö } & NORC -1 & 8.9.2011 & 1,621 & 0,617 & 5,61 & 2,91 & 1,66 \\
\hline & NORC -2 & 8.9 .2011 & 1,664 & 0,601 & 5,46 & 2,29 & 1,60 \\
\hline & NORC -3 & 8.9 .2011 & 1,662 & 0,602 & 5,47 & 2,86 & 1,51 \\
\hline N, Kalkovo & SC10 & 7.9.2009 & 1,291 & 0,775 & 7,04 & 2,08 & 1,22 \\
\hline \multirow{9}{*}{$\mathrm{N}$, Linneset } & sc6-1 & 7.9.2009 & 1,655 & 0,604 & 5,49 & 1,93 & 1,36 \\
\hline & sc6-2 & 7.9.2009 & 1,649 & 0,606 & 5,51 & 1,77 & 1,68 \\
\hline & sc6-3 & 7.9 .2009 & 1,656 & 0,604 & 5,49 & 1,95 & 1,77 \\
\hline & sc6-4 & 7.9.2009 & 1,650 & 0,606 & 5,51 & 2,53 & 2,24 \\
\hline & sc6-5 & 7.9.2009 & 1,674 & 0,597 & 5,43 & 2,24 & 2,28 \\
\hline & sc6-6 & 7.9 .2009 & 1,676 & 0,597 & 5,42 & 2,17 & 1,71 \\
\hline & SC6-7 & 7.9 .2009 & 1,672 & 0,598 & 5,44 & 2,71 & 1,90 \\
\hline & sc6-8 & 7.9 .2009 & 1,654 & 0,605 & 5,50 & 2,59 & 2,40 \\
\hline & SC6-9 & 7.9.2009 & 1,647 & 0,607 & 5,52 & 2,14 & 1,90 \\
\hline $\begin{array}{l}\mathrm{N}, \text { between Formofoss and } \\
\text { Sandmoen }\end{array}$ & SC7 & 7.9 .2009 & 1,283 & 0,779 & 7,08 & 2,33 & 1,68 \\
\hline $\begin{array}{l}\mathrm{N} \text {, between Breidsjøen and } \\
\text { Fofervatnet }\end{array}$ & Sc9 & 7.9.2009 & 1,303 & 0,767 & 6,98 & 2,50 & 2,31 \\
\hline $\mathrm{N}$, between Dovre and Folldal & SC4 & 7.9.2009 & 1,293 & 0,773 & 7,03 & 2,14 & 1,90 \\
\hline $\begin{array}{l}\mathrm{N}, \text { between Lesja and } \\
\text { Skogbygde }\end{array}$ & sc8 & 7.9.2009 & 1,296 & 0,772 & 7,01 & 2,59 & 1,89 \\
\hline \multirow{2}{*}{ N, NP Jotunheimen } & NORB 1 & 5.9 .2011 & 1,277 & 0,783 & 7,12 & 2,72 & 2,31 \\
\hline & NORB 2 & 5.9.2011 & 1,259 & 0,794 & 7,22 & 2,86 & 2,24 \\
\hline \multirow{3}{*}{ N, near NP Rondane } & GPS21 & 10.9 .2008 & 1,277 & 0,783 & 7,12 & 3,00 & 2,48 \\
\hline & GPS25 & 10.9 .2008 & 1,258 & 0,795 & 7,23 & 2,87 & 2,96 \\
\hline & Solkpass & 11.9 .2008 & 1,267 & 0,789 & 7,17 & 2,12 & 2,11 \\
\hline $\mathrm{N}$, Torsefeatn & SC1 & 7.9.2009 & 1,286 & 0,778 & 7,07 & 2,42 & 1,96 \\
\hline \multirow{2}{*}{$\mathrm{N}$, Trollhsimon } & NOR 1 & 5.9 .2011 & 1,278 & 0,782 & 7,11 & 2,99 & 2,25 \\
\hline & NOR 2 & 8.9 .2011 & 1,247 & 0,802 & 7,29 & 2,92 & 1,67 \\
\hline N, Vik & SC2 & 7.9.2009 & 1,287 & 0,777 & 7,06 & 2,35 & 1,62 \\
\hline
\end{tabular}




\section{Numerical Morphometrics}

Central Europe

\begin{tabular}{|c|c|c|c|c|c|c|c|c|c|c|c|c|c|c|c|c|c|c|}
\hline ID & taxa & v2 & v3 & v4 & v5 & v6 & v7 & v8 & v9 & v10 & v11 & v12 & v13 & v14 & v15 & v16 & v17 & GS (2C) \\
\hline KR 1_27 & D. $i$. & 1,4 & 3,0 & 0,5 & 1,8 & 2,1 & 2,9 & 0,6 & 0,6 & 0,9 & 1,8 & 1,4 & 3,3 & 0,7 & 1,5 & 1,9 & 1,2 & 6,01 \\
\hline $2 S ̌ 1$ & D. $t$. & 1,5 & 2,4 & 0,4 & 0,7 & 1,7 & 2,3 & 0,4 & 0,6 & 0,4 & 1,1 & 1,4 & 2,4 & 0,5 & 0,5 & 1,4 & 0,9 & 5,07 \\
\hline 2Š10 & D. $a$. & 2,2 & 2,3 & 0,8 & $-0,2$ & 2,0 & 2,1 & 0,7 & 0,2 & 0,8 & 2,5 & 2,7 & 2,3 & 0,8 & 0,4 & 1,7 & 0,9 & 7,16 \\
\hline 2Š11 & D. $a$. & 1,3 & 2,1 & 0,6 & 0,4 & 1,9 & 2,1 & 0,5 & 0,3 & 0,6 & 1,7 & 1,6 & 2,6 & 0,9 & 0,7 & 1,8 & 0,9 & 7,31 \\
\hline 2Š12 & D. $a$. & 2,1 & 1,9 & 0,8 & 0,0 & 2,2 & 2,0 & 0,6 & 0,2 & 0,8 & 2,3 & 1,1 & 1,6 & 0,8 & 0,4 & 1,7 & 0,9 & 7,32 \\
\hline $25 ̌ 13$ & D. $a$ & 2,4 & 2,5 & 0,7 & 0,1 & 2,1 & 2,5 & 0,6 & 0,4 & 0,8 & 2,5 & 1,4 & 2,2 & 0,8 & 0,5 & 1,7 & 0,8 & 7,41 \\
\hline 2Š14 & D. $a$. & 1,5 & 1,8 & 0,7 & 0,5 & 2,0 & 2,0 & 0,7 & 0,2 & 0,6 & 1,9 & 1,2 & 1,9 & 0,8 & 0,2 & 1,8 & 1,0 & 7,35 \\
\hline 2Š15 & D. $a$ & 1,5 & 2,2 & 0,8 & 0,4 & 2,3 & 2,0 & 0,4 & 0,5 & 0,9 & 2,3 & 1,4 & 2,2 & 0,9 & 0,6 & 1,4 & 0,5 & 7,46 \\
\hline 2 Š2 & D. $a$. & 2,1 & 2,8 & 0,7 & 1,0 & 2,3 & 2,8 & 0,5 & 0,8 & 0,7 & 1,9 & 1,7 & 2,1 & 0,7 & 0,8 & 1,3 & 0,7 & 7,12 \\
\hline 2 Š3 & D. $t$. & 1,3 & 2,0 & 0,3 & 0,6 & 1,3 & 1,8 & 0,5 & 0,6 & 0,4 & 1,4 & 1,5 & 1,9 & 0,5 & 0,5 & 1,6 & 1,1 & 5,12 \\
\hline $2 \check{S} 4$ & D. $a$ & 1,5 & 1,9 & 0,6 & 0,5 & 1,7 & 1,8 & 0,7 & 0,3 & 0,6 & 2,1 & 1,6 & 1,9 & 0,7 & 0,3 & 2,0 & 1,3 & 7,28 \\
\hline 2 Š5 & D. $a$. & 1,9 & 2,1 & 0,7 & $-0,2$ & 1,5 & 1,9 & 0,6 & 0,2 & 0,6 & 1,6 & 2,2 & 1,4 & 0,8 & $-0,5$ & 1,6 & 0,9 & 7,14 \\
\hline 2Š6 & D. $a$. & 2,4 & 2,2 & 0,8 & $-0,3$ & 2,4 & 2,1 & 0,8 & $-0,1$ & 0,5 & 2,4 & 2,0 & 1,9 & 0,9 & 0,2 & 1,9 & 1,1 & 7,22 \\
\hline 2Š8 & D. i. & 2,0 & 2,2 & 0,5 & 0,2 & 1,8 & 2,4 & 0,6 & 0,7 & 0,5 & 1,9 & 2,9 & 1,7 & 0,6 & $-0,3$ & 1,6 & 1,0 & 6,32 \\
\hline 2 šg & D. $a$. & 1,2 & 1,8 & 0,7 & 1,7 & 1,9 & 2,0 & 0,5 & 0,2 & 0,7 & 1,9 & 1,4 & 1,2 & 0,8 & 0,3 & 1,6 & 0,8 & 7,15 \\
\hline 2Š9 & D. $a$. & 1,8 & 1,6 & 0,6 & $-0,4$ & 2,0 & 1,5 & 0,4 & $-0,2$ & 0,5 & 1,8 & 2,2 & 1,5 & 0,5 & $-0,3$ & 1,7 & 1,2 & 7,15 \\
\hline A_1 & D. $a$. & 2,3 & 2,4 & 0,7 & 0,1 & 1,9 & 2,4 & 0,0 & $-1,1$ & 0,8 & 2,1 & 2,9 & 2,1 & 0,7 & 0,7 & 1,5 & 0,8 & 7,10 \\
\hline A_2 & D. a. & 1,9 & 1,9 & 0,8 & $-0,4$ & 2,5 & 2,1 & 0,0 & $-0,5$ & 0,9 & 1,8 & 1,8 & 1,6 & 0,6 & 0,6 & 1,5 & 0,8 & 7,15 \\
\hline A_3 & D. $a$. & 2,3 & 1,8 & 0,8 & $-0,5$ & 1,9 & 1,9 & 0,0 & $-0,3$ & 1,0 & 1,9 & 2,7 & 2,1 & 0,7 & 0,6 & 1,4 & 0,7 & 7,20 \\
\hline ALP 1 & D. $a$. & 1,8 & 1,9 & 0,7 & $-0,1$ & 1,7 & 1,9 & 0,8 & 0,0 & 0,5 & 2,0 & 1,5 & 2,2 & 0,8 & 0,7 & 1,3 & 0,5 & 7,23 \\
\hline BD1 & D. $a$. & 1,8 & 2,9 & 0,6 & 0,5 & 2,2 & 3,0 & 0,5 & 0,9 & 0,6 & 1,9 & 1,6 & 2,3 & 0,7 & 0,3 & 1,7 & 1,8 & 7,28 \\
\hline BD2 & D. $a$. & 2,5 & 2,7 & 0,7 & 0,1 & 2,4 & 3,3 & 0,8 & 1,2 & 0,9 & 2,9 & 2,9 & 2,7 & 0,8 & 0,7 & 1,8 & 1,8 & 7,37 \\
\hline BD3 & D. $a$. & 2,2 & 3,8 & 0,8 & 1,1 & 2,3 & 3,7 & 0,9 & 1,3 & 0,7 & 2,8 & 2,1 & 2,9 & 0,9 & 0,7 & 1,7 & 1,8 & 7,31 \\
\hline BD4 & D. $a$. & 2,0 & 2,2 & 0,7 & 0,3 & 2,1 & 2,4 & 0,7 & 0,2 & 0,6 & 2,0 & 1,3 & 2,0 & 0,9 & 0,3 & 2,0 & 2,1 & 7,28 \\
\hline BD5 & D. $a$. & 2,1 & 2,2 & 0,6 & 0,0 & 2,1 & 2,2 & 0,8 & 0,0 & 0,7 & 2,4 & 1,9 & 2,3 & 0,9 & $-0,2$ & 2,0 & 2,1 & 7,35 \\
\hline BD6 & D. $a$. & 2,1 & 2,8 & 0,5 & 0,4 & 1,9 & 2,1 & 0,6 & 0,6 & 0,5 & 1,9 & 1,4 & 2,9 & 0,7 & 1,1 & 1,5 & 1,5 & 7,35 \\
\hline BEČ 1 & D. $z$. & 2,0 & 3,4 & 0,5 & 1,5 & 2,2 & 3,0 & 1,1 & 0,8 & 0,8 & 2,5 & 1,5 & 2,6 & 0,6 & 0,9 & 2,3 & 2,3 & 5,36 \\
\hline BEČ 10 & D. C. & 1,3 & 2,7 & 0,4 & 1,1 & 1,5 & 1,9 & 0,6 & 0,5 & 0,5 & 2,0 & 1,3 & 2,8 & 0,6 & 1,0 & 2,0 & 2,1 & 5,57 \\
\hline BEČ 11 & D. C. & 1,0 & 2,2 & 0,3 & 1,3 & 0,9 & 2,4 & 0,4 & 1,3 & 0,6 & 2,3 & 1,4 & 1,9 & 0,5 & 0,2 & 1,6 & 1,7 & 5,57 \\
\hline BEČ 12 & D. C. & 1,3 & 1,9 & 0,3 & 0,8 & 1,8 & 2,9 & 0,5 & 0,9 & 0,6 & 2,2 & 1,1 & 2,0 & 0,5 & 0,6 & 1,5 & 1,6 & 5,62 \\
\hline BEČ 13 & D. C. & 1,3 & 2,5 & 0,4 & 0,9 & 1,5 & 2,6 & 0,5 & 1,0 & 0,6 & 2,2 & 1,5 & 2,7 & 0,5 & 0,9 & 1,4 & 1,5 & 5,50 \\
\hline BEČ 2 & D. C. & 1,3 & 2,8 & 0,4 & 1,1 & 1,7 & 3,3 & 0,7 & 1,8 & 0,7 & 2,0 & 1,6 & 3,4 & 0,8 & 1,8 & 1,9 & 2,2 & 5,41 \\
\hline BEČ 3 & D. c. & 1,2 & 2,2 & 0,4 & 1,4 & 1,5 & 2,5 & 0,7 & 0,9 & 0,7 & 2,5 & 1,8 & 2,8 & 0,7 & 1,3 & 2,3 & 2,5 & 5,43 \\
\hline BEČ 4 & D. C. & 1,0 & 1,7 & 0,3 & 0,4 & 1,6 & 1,8 & 0,6 & 0,2 & 0,5 & 2,1 & 1,4 & 1,9 & 0,5 & 0,8 & 1,9 & 2,0 & 5,52 \\
\hline BEČ 5 & D. C. & 1,0 & 2,6 & 0,4 & 1,2 & 1,6 & 2,4 & 0,6 & 0,9 & 0,5 & 1,9 & 1,6 & 2,2 & 0,5 & 0,7 & 1,5 & 1,5 & 5,45 \\
\hline BEČ 6 & D. $i$. & 2,0 & 2,8 & 0,6 & 0,2 & 2,1 & 2,6 & 0,7 & 0,0 & 0,6 & 2,3 & 2,0 & 2,6 & 0,8 & 0,2 & 2,0 & 2,1 & 6,40 \\
\hline BEČ 7 & D. $i$. & 2,0 & 3,1 & 0,8 & 0,8 & 2,1 & 3,0 & 0,9 & 0,5 & 0,6 & 2,1 & 1,9 & 3,2 & 0,9 & 0,7 & 2,3 & 2,3 & 6,42 \\
\hline BEČ 8 & D. C. & 1,5 & 2,8 & 0,4 & 1,3 & 1,9 & 2,9 & 0,9 & 1,1 & 1,0 & 2,1 & 1,8 & 3,1 & 0,8 & 1,3 & 2,6 & 2,8 & 5,39 \\
\hline BEČ 9 & D. c. & 2,1 & 3,0 & 0,4 & 0,2 & 2,2 & 3,3 & 1,1 & 1,0 & 1,0 & 2,7 & 1,9 & 2,1 & 0,5 & 0,5 & 2,4 & 2,5 & 5,59 \\
\hline BES 01 & D. $a$. & 2,4 & 4,3 & 0,5 & 1,8 & 2,1 & 4,2 & 0,5 & 1,9 & 0,5 & 2,1 & 1,9 & 4,5 & 0,6 & 2,0 & 1,6 & 1,7 & 7,36 \\
\hline BES 02 & D. $a$. & 2,2 & 5,0 & 0,8 & 2,5 & 2,2 & 5,2 & 0,8 & 3,0 & 0,7 & 2,8 & 2,5 & 5,5 & 0,8 & 2,2 & 1,9 & 1,9 & 7,37 \\
\hline BES 03 & D. C. & 0,9 & 3,6 & 0,3 & 2,5 & 1,8 & 3,5 & 1,0 & 2,1 & 0,9 & 3,7 & 1,4 & 3,9 & 0,5 & 2,2 & 1,5 & 1,6 & 5,61 \\
\hline BES 04 & D. $a$. & 1,8 & 1,9 & 0,6 & 0,2 & 2,0 & 2,1 & 0,5 & 0,2 & 0,8 & 2,0 & 1,9 & 2,0 & 0,3 & 0,2 & 1,1 & 1,0 & 7,51 \\
\hline BES 05 & D. $a$. & 2,2 & 3,8 & 0,5 & 1,3 & 2,2 & 3,2 & 0,7 & 1,5 & 0,6 & 2,1 & 2,2 & 3,7 & 0,7 & 1,8 & 1,7 & 1,8 & 7,34 \\
\hline
\end{tabular}




\begin{tabular}{|c|c|c|c|c|c|c|c|c|c|c|c|c|c|c|c|c|c|c|}
\hline BES 07 & D. c. & 1,2 & 3,5 & 0,5 & 2,0 & 1,6 & 3,6 & 0,8 & 2,1 & 0,8 & 2,4 & 1,7 & 3,9 & 0,4 & 1,9 & 2,2 & 2,2 & 5,47 \\
\hline BES 08 & D. c. & 0,7 & 2,6 & 0,2 & 2,0 & 1,3 & 2,1 & 0,7 & 1,5 & 0,5 & 2,5 & 1,3 & 2,7 & 0,5 & 1,7 & 1,9 & 2,1 & 5,35 \\
\hline BES 09 & D. $a$. & 2,8 & 4,6 & 0,5 & 2,0 & 2,8 & 4,6 & 0,7 & 2,6 & 0,8 & 3,0 & 2,6 & 4,9 & 0,9 & 2,5 & 1,8 & 2,0 & 7,22 \\
\hline BESK1 & D. c. & 1,0 & 2,7 & 0,3 & 2,8 & 1,8 & 2,6 & 0,9 & 1,5 & 0,9 & 2,4 & 1,9 & 2,9 & 0,7 & 1,2 & 1,8 & 1,0 & 5,48 \\
\hline BESK2 & D.c. & 0,8 & 2,2 & 0,4 & 1,2 & 1,5 & 2,2 & 0,4 & 1,2 & 0,5 & 1,8 & 1,4 & 2,3 & 0,4 & 0,9 & 1,3 & 0,9 & 5,43 \\
\hline BESK3 & D. c. & 0,9 & 2,7 & 0,5 & 1,8 & 1,3 & 3,0 & 0,7 & 1,8 & 0,5 & 1,4 & 1,3 & 2,6 & 0,5 & 1,3 & 1,6 & 1,1 & 5,70 \\
\hline BESK4 & D. c. & 1,2 & 2,7 & 0,3 & 1,8 & 1,5 & 2,9 & 0,8 & 1,8 & 0,8 & 2,2 & 1,5 & 2,8 & 0,5 & 1,3 & 1,5 & 1,0 & 5,45 \\
\hline BESK5 & D. c. & 1,3 & 2,6 & 0,4 & 1,2 & 1,6 & 2,4 & 0,7 & 0,8 & 0,6 & 2,0 & 1,6 & 2,7 & 0,6 & 1,5 & 1,7 & 1,1 & 5,34 \\
\hline Brdy & D.t. & 2,9 & 3,1 & 0,6 & 0,4 & 1,8 & 3,1 & 0,6 & 1,7 & 0,4 & 1,5 & 1,7 & 2,9 & 0,7 & 1,0 & 1,4 & 1,5 & 5,05 \\
\hline BUC 01 & D. $a$. & 2,3 & 2,3 & 0,7 & 0,1 & 2,1 & 2,3 & 0,7 & 0,0 & 0,7 & 2,4 & 2,6 & 2,0 & 0,9 & 0,1 & 1,1 & 1,2 & 7,27 \\
\hline BUC 02 & D. $a$. & 2,4 & 2,8 & 0,7 & 0,6 & 2,3 & 2,7 & 1,1 & 0,6 & 0,7 & 3,2 & 2,0 & 3,0 & 0,8 & 0,3 & 1,1 & 1,2 & 7,04 \\
\hline BUC 03 & D. $a$. & 2,4 & 2,8 & 0,5 & 0,5 & 2,5 & 2,6 & 0,7 & 0,2 & 0,7 & 2,4 & 2,1 & 2,8 & 0,9 & 0,5 & 1,0 & 1,2 & 7,00 \\
\hline BUC 04 & D. $a$. & 2,6 & 2,4 & 0,9 & 0,4 & 2,1 & 2,2 & 0,5 & $-0,1$ & 0,6 & 2,2 & 2,3 & 2,1 & 0,8 & 0,1 & 2,1 & 2,1 & 7,18 \\
\hline BUC 05 & D. $a$. & 2,3 & 2,4 & 0,7 & 0,1 & 2,2 & 2,3 & 0,7 & 0,2 & 0,7 & 2,1 & 2,5 & 2,8 & 0,9 & 0,4 & 1,4 & 1,5 & 7,15 \\
\hline BUC 06 & D. $a$. & 2,3 & 2,9 & 0,4 & 0,6 & 2,2 & 2,9 & 0,7 & 1,0 & 0,8 & 2,8 & 2,0 & 3,3 & 0,8 & 1,1 & 1,0 & 1,2 & 7,23 \\
\hline BUC 07 & D. $a$. & 1,6 & 3,3 & 0,6 & 1,5 & 1,9 & 3,9 & 0,9 & 1,4 & 0,7 & 2,3 & 2,2 & 3,2 & 0,9 & 1,1 & 2,5 & 2,6 & 7,02 \\
\hline BUC 08 & D. $a$. & 1,8 & 1,8 & 0,6 & 0,3 & 1,4 & 1,9 & 0,8 & 0,2 & 0,5 & 2,1 & 1,8 & 1,9 & 0,8 & 0,3 & 2,2 & 2,3 & 6,97 \\
\hline BUC 09 & D. $a$. & 2,2 & 3,2 & 0,7 & 0,5 & 2,6 & 2,7 & 0,8 & 0,3 & 0,7 & 2,4 & 2,2 & 2,6 & 0,8 & 0,5 & 2,3 & 2,4 & 6,91 \\
\hline BUC1 & D. $a$. & 2,3 & 2,7 & 0,8 & 0,4 & 2,1 & 2,4 & 1,0 & 0,2 & 0,6 & 2,1 & 1,7 & 2,6 & 1,0 & 0,7 & 2,8 & 1,8 & 6,70 \\
\hline BUC2 & D. $a$. & 2,2 & 3,4 & 0,8 & 1,2 & 2,5 & 3,7 & 1,1 & 1,4 & 0,6 & 2,4 & 1,9 & 2,6 & 1,0 & 1,0 & 2,9 & 1,9 & 7,01 \\
\hline BUC3 & D. $a$. & 1,9 & 2,3 & 0,5 & 0,2 & 2,1 & 2,2 & 0,7 & 0,4 & 0,5 & 2,2 & 1,9 & 2,5 & 0,7 & 0,4 & 2,7 & 2,0 & 7,32 \\
\hline BUC4 & D. $a$. & 2,8 & 3,3 & 0,9 & 0,4 & 3,2 & 2,9 & 0,6 & 0,5 & 0,7 & 2,7 & 2,2 & 3,5 & 1,0 & 1,2 & 2,8 & 1,8 & 7,14 \\
\hline BUC5 & D. $a$. & 2,0 & 2,0 & 0,6 & 0,1 & 2,0 & 1,7 & 0,6 & 0,0 & 0,6 & 1,7 & 1,6 & 2,1 & 0,6 & 0,2 & 2,2 & 1,6 & 6,43 \\
\hline BUC6 & D. $a$. & 2,7 & 2,9 & 0,8 & 0,3 & 2,5 & 2,8 & 0,7 & 0,3 & 0,7 & 2,5 & 2,3 & 2,9 & 0,9 & 0,7 & 2,5 & 1,6 & 7,29 \\
\hline BUC7 & D. $a$. & 2,1 & 3,2 & 0,7 & 1,1 & 1,8 & 3,2 & 0,8 & 1,6 & 0,7 & 2,1 & 1,9 & 3,6 & 1,0 & 2,1 & 2,6 & 1,7 & 6,58 \\
\hline CBS 01A & D. $i$. & 1,5 & 1,8 & 0,4 & 0,3 & 2,0 & 1,8 & 0,8 & $-0,1$ & 0,6 & 2,5 & 1,4 & 1,8 & 0,8 & 0,1 & 2,3 & 2,5 & 6,57 \\
\hline CBS 01B & D. $i$. & 1,9 & 2,6 & 0,4 & 0,6 & 2,0 & 2,0 & 0,8 & 0,6 & 0,6 & 2,4 & 1,4 & 2,4 & 0,8 & 0,7 & 2,3 & 2,5 & 6,35 \\
\hline CBS 02 & D. $a$. & 2,3 & 2,9 & 0,7 & 0,2 & 2,1 & 2,5 & 0,9 & 0,0 & 0,7 & 2,5 & 2,1 & 2,6 & 0,8 & 0,2 & 1,6 & 1,6 & 7,25 \\
\hline CBS 03 & D. $a$. & 2,7 & 2,2 & 0,8 & $-0,3$ & 2,1 & 2,4 & 0,5 & $-0,1$ & 0,7 & 2,1 & 2,1 & 2,1 & 1,0 & $-0,1$ & 2,2 & 2,3 & 7,25 \\
\hline CBS 04 & D. $i$. & 1,9 & 2,7 & 0,5 & 1,0 & 2,1 & 2,1 & 0,9 & 0,2 & 0,6 & 2,0 & 1,7 & 2,3 & 0,7 & 0,7 & 2,1 & 2,2 & 6,50 \\
\hline CBS 05 & D. c. & 0,9 & 1,8 & 0,3 & 0,8 & 1,1 & 1,7 & 0,6 & 0,5 & 0,5 & 1,7 & 1,2 & 1,7 & 0,6 & 0,4 & 1,9 & 2,0 & 5,49 \\
\hline CBS 06 & D.c. & 1,2 & 2,7 & 0,4 & 1,6 & 1,7 & 2,8 & 0,7 & 1,5 & 0,7 & 2,5 & 1,0 & 2,8 & 0,5 & 1,5 & 2,1 & 2,1 & 5,51 \\
\hline CBS 07 & D. $i$. & 1,6 & 2,8 & 0,5 & 1,1 & 1,8 & 3,0 & 0,9 & 0,6 & 0,6 & 1,7 & 1,7 & 2,8 & 0,9 & 0,7 & 2,2 & 2,4 & 6,33 \\
\hline CBS 09 & D. $a$. & 1,6 & 2,3 & 0,8 & 0,3 & 2,0 & 2,0 & 0,6 & 0,5 & 0,6 & 2,0 & 1,9 & 1,9 & 0,8 & 0,4 & 1,5 & 1,5 & 7,25 \\
\hline CBS 10 & D. c. & 0,6 & 2,3 & 0,4 & 1,5 & 1,7 & 1,8 & 0,5 & 0,4 & 0,7 & 1,7 & 1,3 & 1,8 & 0,5 & 0,7 & 1,6 & 1,7 & 5,56 \\
\hline CER 01 & D. z. & 1,4 & 3,3 & 0,3 & 1,6 & 1,8 & 3,1 & 0,5 & 1,3 & 0,5 & 2,1 & 1,4 & 2,6 & 0,5 & 0,9 & 1,3 & 1,4 & 5,36 \\
\hline CER 02 & D. z. & 1,4 & 3,3 & 0,4 & 2,0 & 1,8 & 3,5 & 0,6 & 1,9 & 0,5 & 1,6 & 1,7 & 3,0 & 0,7 & 1,3 & 1,2 & 1,4 & 5,39 \\
\hline CER 03 & D. c. & 0,5 & 2,9 & 0,4 & 1,5 & 1,5 & 2,2 & 0,8 & 1,2 & 0,5 & 2,3 & 1,7 & 2,8 & 0,7 & 0,6 & 1,3 & 1,4 & 5,55 \\
\hline CER 04 & D. z. & 1,4 & 1,6 & 0,4 & $-0,3$ & 1,3 & 1,2 & 0,4 & $-0,2$ & 0,4 & 1,3 & 1,3 & 1,4 & 0,5 & $-0,1$ & 1,4 & 1,4 & 5,20 \\
\hline CER 05 & D. $i$. & 1,5 & 2,2 & 0,5 & 0,5 & 1,6 & 2,2 & 0,9 & 0,4 & 0,6 & 2,0 & 1,7 & 2,5 & 0,7 & 0,8 & 1,5 & 1,6 & 6,37 \\
\hline CPL 01 & D. $a$. & 2,3 & 3,0 & 0,8 & 0,4 & 2,3 & 3,1 & 0,9 & 1,4 & 1,0 & 2,8 & 2,2 & 3,1 & 1,0 & 0,4 & 1,5 & 1,6 & 7,16 \\
\hline CPL 02 & D. $a$. & 1,9 & 2,3 & 0,6 & 0,1 & 2,0 & 2,2 & 0,8 & 0,6 & 0,7 & 2,7 & 1,5 & 2,2 & 0,8 & 0,1 & 1,6 & 1,7 & 7,20 \\
\hline CPL 03 & D. $a$. & 2,4 & 2,3 & 0,7 & $-0,3$ & 2,2 & 2,3 & 0,8 & 0,1 & 0,8 & 2,4 & 2,4 & 2,5 & 0,7 & $-0,2$ & 1,3 & 1,3 & 7,16 \\
\hline CPL 04 & D. $a$. & 2,9 & 2,8 & 0,9 & $-0,1$ & 2,9 & 2,9 & 0,4 & 0,5 & 1,0 & 2,5 & 2,1 & 2,9 & 0,9 & 0,4 & 1,6 & 1,6 & 7,12 \\
\hline CPL 05 & D. $a$. & 0,9 & 1,4 & 0,6 & 0,0 & 2,0 & 1,4 & 0,7 & $-0,3$ & 0,6 & 2,1 & 1,4 & 1,3 & 0,7 & $-0,1$ & 2,2 & 2,3 & 7,21 \\
\hline CPL 06 & D. $a$. & 1,1 & 1,3 & 0,5 & $-0,3$ & 1,7 & 1,2 & 0,5 & $-0,3$ & 0,6 & 1,9 & 1,3 & 1,0 & 0,5 & $-0,5$ & 1,6 & 1,6 & 7,21 \\
\hline CRU 01 & . c. & 0,9 & 3,1 & 0,5 & 1,5 & 1,4 & 3,0 & 0,6 & 1,7 & 0,7 & 2,0 & 1,4 & 2,6 & 0,6 & 0,9 & 1,8 & 1,9 & 5,54 \\
\hline CRU 02 & D. c. & 0,5 & 2,6 & 0,5 & 2,0 & 1,3 & 2,7 & 0,6 & 1,9 & 0,4 & 2,3 & 1,4 & 2,6 & 0,6 & 1,3 & 1,7 & 1,8 & 5,52 \\
\hline CRU 03 & D. C. & 0,9 & 3,0 & 0,4 & 1,9 & 1,0 & 2,7 & 0,6 & 1,5 & 0,6 & 2,0 & 1,2 & 3,3 & 0,6 & 2,3 & 1,9 & 2,0 & 5,57 \\
\hline CRU 04 & D. C. & 0,7 & 3,2 & 0,3 & 1,9 & 1,7 & 2,9 & 0,3 & 1,7 & 0,5 & 2,7 & 1,1 & 2,9 & 0,4 & 1,9 & 1,8 & 1,8 & 5,47 \\
\hline
\end{tabular}




\begin{tabular}{|c|c|c|c|c|c|c|c|c|c|c|c|c|c|c|c|c|c|c|}
\hline CRU 05 & D. c. & 0,9 & 2,8 & 0,4 & 1,9 & 1,5 & 2,9 & 0,6 & 1,5 & 0,6 & 2,9 & 1,3 & 2,7 & 0,7 & 1,1 & 1,9 & 2,1 & 5,61 \\
\hline CRU 06 & D. c. & 0,6 & 3,3 & 0,3 & 2,7 & 1,4 & 3,8 & 0,8 & 2,9 & 0,6 & 2,4 & 1,1 & 3,2 & 0,5 & 2,2 & 1,9 & 2,0 & 5,69 \\
\hline HB1 & D. $i$. & 2,5 & 5,0 & 0,7 & 2,8 & 2,3 & 4,1 & 1,3 & 1,9 & 0,7 & 2,7 & 1,6 & 5,0 & 1,1 & 2,4 & 2,8 & 3,0 & 6,30 \\
\hline HB2 & D. $i$. & 0,9 & 3,0 & 0,7 & 0,9 & 1,7 & 2,4 & 0,8 & 0,7 & 0,8 & 2,3 & 1,7 & 4,9 & 1,2 & 2,6 & 2,6 & 2,9 & 6,42 \\
\hline HB3 & D. $i$. & 1,7 & 3,0 & 0,7 & 0,7 & 1,7 & 2,8 & 0,8 & 1,1 & 0,8 & 2,4 & 2,2 & 2,9 & 0,8 & 1,5 & 2,3 & 2,4 & 6,38 \\
\hline HUR 01 & D. $a$. & 2,3 & 2,0 & 0,7 & $-0,3$ & 2,1 & 1,9 & 0,5 & $-0,2$ & 0,6 & 2,5 & 1,9 & 1,5 & 0,8 & 0,0 & 2,1 & 2,2 & 7,18 \\
\hline HUR 02 & D. $a$. & 1,9 & 1,9 & 0,6 & 0,2 & 2,1 & 2,1 & 0,8 & 0,0 & 0,6 & 2,4 & 1,7 & 2,0 & 0,8 & 0,0 & 2,2 & 2,3 & 6,86 \\
\hline HUR 03 & D. $a$. & 2,3 & 2,7 & 0,5 & 0,8 & 2,1 & 2,6 & 0,7 & 0,6 & 0,6 & 2,6 & 1,8 & 2,7 & 0,7 & 0,6 & 1,0 & 1,1 & 7,34 \\
\hline Je 1_1 & D. $a$. & 1,9 & 2,4 & 0,6 & 0,6 & 2,1 & 2,6 & 0,8 & 0,4 & 0,8 & 2,5 & 2,2 & 2,5 & 0,8 & 0,2 & 1,0 & 1,1 & 7,68 \\
\hline Je 1_2 & D. $a$. & 2,4 & 3,0 & 0,6 & 0,5 & 2,0 & 2,7 & 0,6 & 0,8 & 0,6 & 2,0 & 2,2 & 2,8 & 0,7 & 1,1 & 1,3 & 1,3 & 7,61 \\
\hline Je 1_3 & D. $a$. & 2,5 & 5,9 & 0,6 & 2,7 & 2,6 & 5,6 & 0,6 & 3,4 & 0,7 & 2,8 & 2,1 & 6,1 & 0,8 & 3,1 & 1,0 & 1,1 & 7,67 \\
\hline Je 1_4 & D. $a$. & 2,4 & 3,2 & 0,6 & 0,7 & 2,1 & 3,4 & 0,9 & 0,8 & 0,9 & 2,4 & 1,8 & 2,8 & 0,8 & 1,0 & 1,3 & 1,4 & 7,44 \\
\hline Je 2_1 & D. $a$. & 2,4 & 1,6 & 0,9 & $-0,6$ & 1,8 & 1,6 & 0,5 & $-0,3$ & 0,9 & 2,1 & 2,4 & 1,8 & 0,9 & $-0,5$ & 1,7 & 1,7 & 7,66 \\
\hline Je 3_1 & D. $a$. & 2,2 & 2,7 & 0,8 & 0,4 & 2,0 & 2,5 & 0,4 & 0,3 & 0,7 & 1,9 & 1,9 & 2,4 & 0,8 & 0,3 & 0,9 & 0,9 & 7,65 \\
\hline Je 3_2 & D. $a$. & 2,8 & 3,0 & 0,9 & 0,5 & 3,3 & 2,9 & 0,5 & $-0,3$ & 0,8 & 1,5 & 3,0 & 3,2 & 0,8 & 0,3 & 1,4 & 1,3 & 7,40 \\
\hline Je 3_3 & D. $a$. & 2,3 & 2,3 & 0,8 & 0,0 & 2,2 & 2,1 & 0,4 & 0,0 & 0,7 & 1,7 & 2,5 & 2,3 & 0,8 & $-0,2$ & 1,4 & 1,5 & 7,57 \\
\hline Je 3_4 & D. $a$. & 2,6 & 3,8 & 1,2 & 1,3 & 2,4 & 3,5 & 0,4 & 1,6 & 0,8 & 2,1 & 2,2 & 3,2 & 1,0 & 1,4 & 1,3 & 1,2 & 7,37 \\
\hline Je 4_1 & D. $a$. & 1,9 & 2,2 & 0,7 & 0,3 & 2,0 & 2,4 & 0,5 & 0,4 & 0,7 & 1,8 & 1,7 & 2,4 & 0,6 & 0,6 & 1,1 & 1,1 & 7,49 \\
\hline Je 4_2 & D. $a$. & 2,1 & 2,0 & 0,8 & $-0,1$ & 1,8 & 2,0 & 0,6 & $-0,1$ & 0,7 & 2,1 & 1,9 & 1,6 & 0,7 & $-0,3$ & 0,8 & 0,8 & 7,49 \\
\hline Je 5_1 & D. $a$. & 2,6 & 3,0 & 0,8 & 0,8 & 2,7 & 3,3 & 1,0 & 0,9 & 1,0 & 2,7 & 2,3 & 2,8 & 1,0 & 0,8 & 3,1 & 3,2 & 7,25 \\
\hline Je 5_2 & D. $a$. & 2,1 & 2,6 & 0,8 & 0,3 & 2,1 & 2,5 & 0,4 & 0,3 & 0,6 & 2,1 & 2,0 & 3,0 & 0,9 & 0,5 & 1,5 & 1,6 & 7,41 \\
\hline Je 5_3 & D. $a$. & 2,3 & 2,9 & 0,8 & 0,2 & 2,3 & 2,4 & 0,8 & 0,4 & 0,8 & 2,5 & 2,3 & 2,6 & 1,0 & 0,0 & 2,3 & 2,4 & 7,52 \\
\hline Je 5_4 & D. $a$. & 2,0 & 2,6 & 0,9 & 0,2 & 2,3 & 2,6 & 0,5 & 0,0 & 0,6 & 2,3 & 2,5 & 2,5 & 1,0 & 0,1 & 1,9 & 1,9 & 7,39 \\
\hline Je 5_6 & D. $a$. & 1,8 & 2,1 & 0,8 & 0,1 & 1,9 & 2,3 & 0,4 & 0,4 & 0,6 & 1,7 & 2,1 & 2,2 & 0,8 & $-0,2$ & 1,5 & 1,5 & 7,34 \\
\hline Je 6_1 & D. a. & 2,3 & 3,7 & 0,5 & 1,3 & 2,3 & 3,7 & 0,8 & 1,6 & 0,9 & 2,8 & 1,7 & 3,4 & 0,7 & 2,0 & 0,9 & 1,0 & 7,48 \\
\hline Je 6_10 & D. $a$. & 2,6 & 3,8 & 0,6 & 1,4 & 2,3 & 3,3 & 0,8 & 1,3 & 0,7 & 2,7 & 2,0 & 3,4 & 0,8 & 2,0 & 1,5 & 1,6 & 7,58 \\
\hline Je 6_2 & D. $a$. & 2,4 & 2,6 & 0,9 & 0,0 & 2,4 & 2,7 & 0,7 & 0,3 & 0,7 & 2,5 & 2,6 & 2,7 & 0,9 & 0,6 & 1,3 & 1,3 & 7,38 \\
\hline Je 6_3 & D. $a$. & 2,1 & 2,3 & 0,6 & 0,0 & 2,0 & 2,6 & 0,5 & 0,4 & 0,6 & 2,0 & 2,5 & 2,6 & 0,9 & 0,4 & 1,7 & 1,9 & 7,46 \\
\hline Je 6_4 & D. $a$. & 1,5 & 1,4 & 0,6 & $-0,2$ & 1,8 & 1,4 & 0,5 & $-0,1$ & 0,6 & 2,4 & 1,5 & 1,6 & 0,7 & 0,0 & 1,7 & 1,7 & 7,21 \\
\hline Je 6_5 & D. $a$. & 2,5 & 3,8 & 0,7 & 1,4 & 2,3 & 3,8 & 0,8 & 1,2 & 0,8 & 3,2 & 2,3 & 3,9 & 0,9 & 1,6 & 1,4 & 1,5 & 7,30 \\
\hline Je 6_6 & D. $a$. & 2,1 & 2,6 & 0,7 & 0,4 & 2,0 & 2,3 & 0,7 & 0,9 & 0,8 & 2,8 & 1,9 & 2,3 & 0,8 & 1,2 & 1,1 & 1,1 & 7,43 \\
\hline Je 6_7 & D. $a$. & 2,3 & 2,4 & 0,7 & 0,1 & 2,3 & 2,6 & 0,8 & 0,0 & 0,7 & 2,8 & 2,3 & 2,8 & 0,9 & 0,5 & 2,5 & 2,6 & 7,40 \\
\hline Je 6_8 & D. $a$. & 1,7 & 2,0 & 0,7 & 0,3 & 1,9 & 1,9 & 0,4 & 0,4 & 0,7 & 2,4 & 1,9 & 2,0 & 0,7 & 0,0 & 0,9 & 0,9 & 7,47 \\
\hline Je 6_9 & D. $a$. & 2,0 & 2,8 & 0,7 & 0,5 & 2,4 & 2,7 & 0,5 & 0,5 & 0,8 & 2,4 & 2,0 & 2,5 & 0,8 & 0,6 & 1,1 & 1,2 & 7,59 \\
\hline K1 & D.t. & 1,4 & 2,1 & 0,6 & 0,7 & 1,4 & 2,0 & 0,0 & 0,4 & 0,4 & 1,5 & 1,4 & 8,6 & 0,6 & 0,5 & 1,5 & 0,8 & 4,86 \\
\hline K2 & D. C. & 0,5 & 2,4 & 0,3 & 2,0 & 1,2 & 2,7 & 0,0 & 1,2 & 0,8 & 2,1 & 1,5 & 2,5 & 0,5 & 1,0 & 1,7 & 1,2 & 5,35 \\
\hline K3 & D.t. & 1,7 & 1,8 & 0,6 & 0,7 & 1,3 & 2,5 & 0,0 & 0,4 & 0,4 & 1,3 & 1,4 & 1,9 & 0,7 & 0,6 & 1,5 & 0,8 & 4,85 \\
\hline K4 & D.t. & 1,1 & 2,7 & 0,5 & 1,2 & 1,4 & 2,8 & 0,0 & 0,9 & 0,5 & 1,1 & 1,3 & 2,1 & 0,7 & 1,2 & 1,3 & 0,6 & 4,93 \\
\hline K5 & D. C. & 1,3 & 3,3 & 0,5 & 2,5 & 1,5 & 3,4 & 0,0 & 1,5 & 0,9 & 2,6 & 1,4 & 3,1 & 0,6 & 0,8 & 2,1 & 1,5 & 5,48 \\
\hline K6 & D.t. & 1,2 & 2,5 & 0,5 & 1,1 & 1,4 & 2,4 & 0,0 & 0,7 & 0,4 & 1,4 & 1,2 & 2,4 & 0,6 & 0,8 & 1,3 & 0,7 & 4,85 \\
\hline K7 & D.t. & 1,2 & 2,3 & 0,4 & 1,0 & 1,3 & 2,5 & 0,0 & 1,1 & 0,4 & 1,3 & 1,4 & 2,3 & 0,3 & 1,3 & 1,1 & 0,8 & 4,93 \\
\hline K8 & D.t. & 1,2 & 1,9 & 0,4 & 0,6 & 1,3 & 2,3 & 0,0 & 0,9 & 0,3 & 1,3 & 0,9 & 1,9 & 0,4 & 0,6 & 1,1 & 0,6 & 4,99 \\
\hline KAL 1 & D. c. & 1,0 & 1,9 & 0,6 & 0,7 & 1,4 & 1,5 & 0,6 & 0,4 & 0,7 & 1,8 & 1,4 & 2,1 & 0,7 & 0,3 & 2,0 & 1,3 & 5,43 \\
\hline KAL 2 & D.c. & 1,0 & 2,2 & 0,5 & 1,0 & 1,3 & 2,3 & 0,4 & 0,7 & 0,6 & 2,2 & 1,3 & 1,9 & 0,5 & 0,6 & 1,8 & 1,2 & 5,39 \\
\hline KB 1 & D.t. & 1,3 & 2,1 & 0,5 & 0,9 & 1,4 & 2,2 & 0,4 & 0,7 & 0,4 & 1,4 & 1,5 & 2,2 & 0,5 & 0,8 & 1,3 & 0,9 & 4,87 \\
\hline KB 10 & D.t. & 1,4 & 2,6 & 0,4 & 1,2 & 1,4 & 2,8 & 0,3 & 1,3 & 0,4 & 1,3 & 1,4 & 3,0 & 0,7 & 1,5 & 1,5 & 0,8 & 4,87 \\
\hline KB 11 & D.t. & 1,6 & 3,9 & 0,3 & 2,2 & 1,6 & 3,4 & 0,3 & 1,7 & 0,5 & 0,9 & 1,4 & 3,5 & 0,4 & 2,1 & 1,4 & 1,0 & 4,83 \\
\hline KB 12 & D. c. & 0,5 & 2,0 & 0,4 & 1,3 & 1,3 & 2,1 & 0,5 & 1,0 & 0,5 & 2,0 & 1,2 & 2,6 & 0,6 & 1,3 & 1,6 & 1,0 & 5,46 \\
\hline KB 13 & D.t. & 1,3 & 2,2 & 0,5 & 0,7 & 1,5 & 2,2 & 0,4 & 0,7 & 0,4 & 1,2 & 1,5 & 2,3 & 0,6 & 0,8 & 1,5 & 0,8 & 4,73 \\
\hline
\end{tabular}




\begin{tabular}{|c|c|c|c|c|c|c|c|c|c|c|c|c|c|c|c|c|c|c|}
\hline KB 14 & D. $t$. & 1,4 & 2,1 & 0,5 & 0,8 & 1,4 & 2,3 & 0,3 & 1,1 & 0,4 & 1,3 & 1,4 & 2,4 & 0,7 & 1,3 & 1,1 & 0,5 & 4,95 \\
\hline KB 15 & D. $t$. & 1,5 & 3,2 & 0,7 & 1,7 & 1,5 & 3,0 & 0,3 & 1,5 & 0,5 & 1,2 & 1,5 & 2,9 & 0,8 & 1,6 & 1,4 & 0,6 & 4,92 \\
\hline KB 17 & D.t. & 1,3 & 2,6 & 0,5 & 1,2 & 1,6 & 2,9 & 0,4 & 1,4 & 0,4 & 1,2 & 1,3 & 2,8 & 0,6 & 1,4 & 1,4 & 0,7 & 4,99 \\
\hline KB 18 & D. c. & 0,8 & 3,1 & 0,2 & 2,4 & 1,5 & 3,2 & 0,4 & 1,9 & 1,0 & 2,5 & 1,2 & 3,0 & 0,8 & 1,3 & 2,0 & 1,2 & 5,56 \\
\hline KB 19 & D.c. & 0,8 & 2,9 & 0,4 & 1,8 & 1,4 & 2,9 & 0,4 & 1,7 & 0,7 & 2,1 & 1,4 & 3,0 & 0,8 & 1,8 & 2,0 & 1,1 & 5,47 \\
\hline KB 2 & D. c. & 0,9 & 2,9 & 0,3 & 1,4 & 1,5 & 2,9 & 0,7 & 1,6 & 0,9 & 2,6 & 1,2 & 3,6 & 0,7 & 2,1 & 2,2 & 1,5 & 5,45 \\
\hline KB 20 & D. c. & 0,8 & 2,9 & 0,3 & 1,7 & 1,4 & 2,1 & 0,3 & 0,9 & 0,8 & 2,3 & 1,1 & 2,4 & 0,6 & 0,9 & 1,7 & 1,1 & 5,53 \\
\hline KB 21 & D. C. & 0,7 & 2,6 & 0,3 & 1,8 & 1,2 & 2,8 & 0,3 & 1,7 & 0,5 & 1,7 & 1,3 & 2,5 & 0,6 & 1,2 & 1,5 & 0,9 & 5,71 \\
\hline KB 22 & D. C. & 0,9 & 1,8 & 0,3 & 0,9 & 1,2 & 1,8 & 0,6 & 0,7 & 0,5 & 2,1 & 1,3 & 1,6 & 0,7 & 0,6 & 1,8 & 1,1 & 5,49 \\
\hline KB 23 & D.t. & 1,3 & 2,2 & 0,6 & 0,8 & 1,3 & 2,0 & 0,4 & 0,7 & 0,4 & 1,1 & 1,3 & 2,2 & 0,3 & 1,0 & 1,4 & 1,1 & 4,95 \\
\hline KB 24 & D.t. & 1,3 & 2,3 & 0,5 & 1,2 & 1,5 & 2,3 & 0,3 & 0,6 & 0,4 & 1,3 & 1,3 & 2,5 & 0,5 & 2,4 & 1,3 & 0,7 & 4,96 \\
\hline KB 25 & D. C. & 0,8 & 2,8 & 0,3 & 1,6 & 1,2 & 3,1 & 0,6 & 1,4 & 0,5 & 1,8 & 1,5 & 2,5 & 0,6 & 1,0 & 1,9 & 1,3 & 5,56 \\
\hline KB 26 & D.t. & 1,4 & 2,3 & 0,5 & 1,1 & 1,3 & 2,3 & 0,4 & 1,1 & 0,4 & 1,1 & 1,6 & 2,2 & 0,8 & 0,9 & 1,4 & 0,7 & 4,97 \\
\hline KB 27 & D. c. & 0,7 & 1,9 & 0,2 & 1,1 & 1,4 & 2,1 & 0,3 & 0,9 & 0,5 & 1,7 & 1,4 & 2,2 & 0,7 & 0,8 & 1,6 & 1,0 & 5,46 \\
\hline KB 28 & D.t. & 1,3 & 2,8 & 0,4 & 1,8 & 1,5 & 3,2 & 0,3 & 1,7 & 0,4 & 1,1 & 1,3 & 3,5 & 0,5 & 1,9 & 1,2 & 0,8 & 5,02 \\
\hline KB 29 & D.t. & 1,5 & 2,6 & 0,4 & 1,2 & 1,2 & 2,2 & 0,4 & 0,9 & 0,4 & 1,4 & 1,4 & 2,1 & 0,7 & 0,8 & 1,4 & 0,7 & 5,08 \\
\hline KB 30 & D. C. & 0,9 & 2,2 & 0,3 & 1,3 & 1,2 & 1,7 & 0,5 & 0,7 & 0,6 & 1,8 & 1,2 & 1,9 & 0,5 & 0,8 & 1,8 & 1,2 & 5,08 \\
\hline KB 4 & D.t. & 1,4 & 2,9 & 0,5 & 1,2 & 1,5 & 2,8 & 0,5 & 1,4 & 0,4 & 1,4 & 1,5 & 2,8 & 0,7 & 1,4 & 1,6 & 0,9 & 5,04 \\
\hline KB 5 & D. C. & 0,6 & 2,1 & 0,3 & 1,3 & 1,2 & 1,9 & 0,3 & 0,9 & 0,5 & 1,5 & 1,1 & 1,7 & 0,5 & 0,6 & 1,5 & 1,0 & 5,46 \\
\hline KB 6 & D. z. & 1,6 & 3,8 & 0,4 & 1,8 & 1,6 & 3,5 & 0,5 & 2,1 & 0,5 & 1,7 & 1,5 & 3,9 & 0,5 & 2,4 & 1,9 & 1,4 & 4,88 \\
\hline KB 7 & D. C. & 0,9 & 3,3 & 0,3 & 2,1 & 0,8 & 3,1 & 0,2 & 2,1 & 1,1 & 2,1 & 1,6 & 2,9 & 0,6 & 1,2 & 1,6 & 0,9 & 5,41 \\
\hline KB 8 & D.t. & 1,4 & 2,6 & 0,5 & 1,1 & 1,4 & 2,5 & 0,3 & 0,9 & 0,4 & 1,3 & 1,2 & 2,2 & 0,6 & 0,8 & 1,4 & 0,7 & 4,99 \\
\hline KB 9 & D. c. & 1,0 & 3,4 & 0,3 & 1,9 & 1,7 & 3,4 & 0,5 & 2,2 & 0,9 & 2,5 & 1,4 & 3,6 & 0,8 & 2,0 & 2,1 & 1,3 & 5,48 \\
\hline KLA 01 & D. C. & 1,0 & 2,0 & 0,3 & 1,3 & 1,5 & 2,1 & 0,7 & 1,1 & 0,4 & 1,6 & 1,2 & 2,2 & 0,8 & 0,9 & 1,7 & 1,9 & 5,66 \\
\hline KLA 02 & D. c. & 1,1 & 2,1 & 0,5 & 1,3 & 1,6 & 2,1 & 0,6 & 0,7 & 0,8 & 2,3 & 1,2 & 1,6 & 0,4 & 0,2 & 1,5 & 1,5 & 5,58 \\
\hline KLA 03 & D. c. & 0,9 & 2,2 & 0,4 & 1,2 & 1,4 & 1,9 & 0,7 & 1,0 & 0,6 & 1,9 & 1,2 & 2,0 & 0,4 & 1,1 & 1,6 & 1,5 & 5,57 \\
\hline KLA 04 & D. C. & 0,9 & 2,3 & 0,5 & 1,4 & 1,0 & 2,3 & 0,5 & 1,5 & 0,4 & 1,6 & 1,4 & 2,2 & 0,5 & 1,3 & 1,5 & 1,5 & 5,66 \\
\hline KLA 05 & D. C. & 1,0 & 3,1 & 0,4 & 2,1 & 1,5 & 3,3 & 0,9 & 1,8 & 0,6 & 2,0 & 1,5 & 2,7 & 0,9 & 1,6 & 1,8 & 2,1 & 5,67 \\
\hline KLA 06 & D. c. & 0,8 & 2,5 & 0,4 & 1,5 & 1,3 & 3,0 & 0,8 & 1,5 & 0,5 & 1,9 & 1,1 & 1,5 & 0,6 & 1,3 & 1,6 & 1,7 & 5,67 \\
\hline KLA 07 & D. c. & 0,6 & 1,7 & 0,4 & 1,7 & 1,1 & 2,2 & 0,6 & 1,2 & 0,5 & 2,1 & 1,3 & 2,2 & 0,5 & 1,0 & 1,8 & 1,9 & 5,53 \\
\hline KLA 08 & D. C & 1,0 & 3,2 & 0,3 & 2,3 & 1,4 & 3,2 & 0,7 & 2,1 & 0,7 & 2,3 & 1,4 & 2,7 & 0,8 & 1,5 & 1,8 & 2,1 & 5,67 \\
\hline KLA 09 & D.c. & 1,1 & 3,0 & 0,4 & 1,9 & 1,4 & 3,1 & 0,7 & 1,9 & 0,5 & 1,8 & 1,1 & 2,4 & 0,7 & 1,3 & 1,6 & 1,7 & 5,71 \\
\hline KLA 10 & D. c. & 0,7 & 2,0 & 0,4 & 1,5 & 1,1 & 2,3 & 0,6 & 1,1 & 0,5 & 1,4 & 1,2 & 3,1 & 0,7 & 1,8 & 1,8 & 1,9 & 5,60 \\
\hline KLA1 & D. C. & 3,3 & 3,2 & 0,4 & 1,8 & 1,2 & 2,5 & 2,1 & 1,5 & 0,6 & 2,0 & 1,4 & 2,6 & 0,7 & 1,6 & 2,0 & 1,3 & 5,55 \\
\hline KLA2 & D. C. & 1,1 & 2,5 & 0,4 & 1,3 & 1,4 & 2,1 & 0,7 & 0,7 & 0,7 & 2,2 & 1,1 & 2,4 & 0,6 & 1,3 & 2,1 & 1,5 & 65 \\
\hline KLA3 & D. C. & 1,1 & 2,2 & 0,5 & 1,6 & 1,4 & 2,3 & 0,7 & 0,8 & 0,7 & 2,3 & 1,1 & 2,1 & 0,7 & 0,7 & 2,3 & 1,6 & 5,57 \\
\hline KLA4 & D. c. & 1,4 & 2,3 & 0,5 & 0,7 & 1,4 & 1,6 & 0,7 & 0,4 & 0,6 & 1,8 & 1,3 & 1,8 & 0,7 & 0,5 & 2,2 & 1,5 & 5,59 \\
\hline KLA5 & D. c. & 1,1 & 1,5 & 0,4 & 0,9 & 1,7 & 1,8 & 0,9 & 0,9 & 0,7 & 3,2 & 1,2 & 2,8 & 0,5 & 1,2 & 2,6 & 2,1 & 5,51 \\
\hline KNI 01 & $o$. & 2,1 & 2,2 & 0,5 & $-0,1$ & 2,3 & 2,1 & 0,9 & $-0,1$ & 0,4 & 2,6 & 1,9 & 1,9 & 0,7 & $-0,2$ & 2,6 & 2,7 & 6,37 \\
\hline KNI 02 & D. $a$. & 1,9 & 1,7 & 0,5 & $-0,3$ & 2,0 & 1,8 & 0,5 & $-0,1$ & 0,9 & 2,3 & 1,9 & 1,6 & 0,7 & $-0,3$ & 1,5 & 1,6 & 7,19 \\
\hline KNI 03 & D.t. & 1,3 & 1,9 & 0,3 & 0,7 & 1,5 & 2,2 & 0,4 & 1,1 & 0,3 & 0,9 & 1,4 & 1,9 & 0,7 & 1,0 & 1,3 & 1,5 & 5,31 \\
\hline KNI 04 & D.t. & 1,3 & 2,4 & 0,5 & 0,7 & 1,6 & 2,4 & 0,4 & 0,6 & 0,3 & 0,8 & 1,4 & 2,5 & 0,7 & 0,8 & 1,4 & 1,5 & 5,04 \\
\hline KNI 05 & D.t. & 1,5 & 2,7 & 0,5 & 1,0 & 1,7 & 2,4 & 0,5 & 0,7 & 0,4 & 1,1 & 1,4 & 2,6 & 0,6 & 1,4 & 1,3 & 1,4 & 5,01 \\
\hline KNI 06 & D.t. & 1,6 & 2,1 & 0,6 & 0,7 & 1,4 & 2,2 & 0,5 & 0,5 & 0,3 & 1,4 & 1,4 & 2,6 & 0,6 & 1,1 & 1,3 & 1,4 & 5,02 \\
\hline KNI 07 & D. a. & 1,7 & 1,8 & 0,7 & $-0,2$ & 1,6 & 1,7 & 0,5 & $-0,1$ & 0,8 & 2,3 & 1,9 & 1,6 & 0,8 & $-0,2$ & 2,2 & 2,2 & 7,19 \\
\hline KNI 08 & D. $a$. & 2,5 & 1,9 & 0,8 & $-0,2$ & 2,2 & 2,1 & 0,6 & 0,0 & 0,7 & 1,7 & 2,3 & 2,8 & 1,0 & 0,6 & 2,1 & 2,2 & 7,25 \\
\hline KNI 09 & D. $a$. & 1,7 & 1,6 & 0,6 & $-0,2$ & 1,5 & 1,7 & 0,6 & $-0,1$ & 0,6 & 2,0 & 1,7 & 1,5 & 0,6 & $-0,3$ & 1,9 & 1,9 & 7,38 \\
\hline KNI 10 & D. $a$. & 2,3 & 2,2 & 1,0 & $-0,2$ & 1,9 & 2,0 & 0,6 & $-0,3$ & 0,8 & 2,3 & 2,2 & 2,3 & 1,0 & $-0,5$ & 1,8 & 1,8 & 7,32 \\
\hline KR 1_10 & D. C. & 1,5 & 3,0 & 0,5 & 1,6 & 2,6 & 3,0 & 0,4 & 0,6 & 0,6 & 1,9 & 2,0 & 3,1 & 0,6 & 1,2 & 1,5 & 0,9 & 5,95 \\
\hline KR 1_11 & D.t. & 1,7 & 2,6 & 0,3 & 0,8 & 1,7 & 2,4 & 0,3 & 0,9 & 0,4 & 1,7 & 1,5 & 2,7 & 0,4 & 0,9 & 1,5 & 1,2 & 4,90 \\
\hline
\end{tabular}




\begin{tabular}{|c|c|c|c|c|c|c|c|c|c|c|c|c|c|c|c|c|c|c|}
\hline KR 1_12 & D. $t$. & 1,2 & 1,9 & 0,4 & 0,7 & 1,3 & 1,9 & 0,4 & 0,7 & 0,2 & 0,8 & 1,3 & 1,9 & 0,6 & 0,6 & 1,0 & 0,5 & 4,87 \\
\hline KR 1_13 & D. $z$. & 1,6 & 2,0 & 0,3 & 0,7 & 1,3 & 1,8 & 0,5 & 0,5 & 0,6 & 2,2 & 1,8 & 2,1 & 0,5 & 0,3 & 1,8 & 1,4 & 5,28 \\
\hline KR 1_14 & D. t. & 1,5 & 2,5 & 0,4 & 0,8 & 1,6 & 2,6 & 0,4 & 1,1 & 0,6 & 0,7 & 2,1 & 2,3 & 0,6 & 0,6 & 1,5 & 0,9 & 4,91 \\
\hline KR 1_15 & D. $i$. & 1,4 & 2,5 & 0,5 & 0,8 & 1,9 & 2,6 & 0,6 & 0,6 & 0,4 & 2,1 & 1,6 & 2,2 & 0,5 & 0,7 & 1,6 & 1,1 & 6,04 \\
\hline KR 1_16 & D. $t$. & 1,1 & 2,1 & 0,6 & 1,1 & 1,4 & 2,0 & 0,3 & 0,6 & 0,3 & 1,5 & 1,1 & 2,5 & 0,6 & 0,8 & 1,4 & 0,7 & 4,87 \\
\hline KR 1_17 & D. $i$. & 2,0 & 2,8 & 0,4 & 1,0 & 1,7 & 3,0 & 0,4 & 0,7 & 0,7 & 1,9 & 1,9 & 2,5 & 0,8 & 0,9 & 1,8 & 1,0 & 5,97 \\
\hline KR 1_18 & D. $a$. & 2,6 & 3,4 & 0,8 & 0,8 & 2,4 & 3,6 & 0,6 & 1,3 & 0,8 & 2,4 & 2,4 & 3,9 & 0,6 & 1,3 & 1,6 & 1,0 & 7,05 \\
\hline KR 1_19 & D. $t$. & 1,5 & 2,5 & 0,3 & 1,2 & 1,5 & 2,8 & 0,3 & 1,0 & 0,4 & 1,5 & 1,3 & 2,3 & 0,5 & 0,9 & 1,5 & 1,0 & 4,84 \\
\hline KR 1_2 & D. C. & 1,6 & 4,3 & 0,5 & 2,6 & 1,6 & 4,0 & 0,6 & 2,7 & 0,9 & 2,9 & 1,5 & 3,5 & 0,9 & 1,9 & 2,2 & 1,3 & 5,41 \\
\hline KR 1_20 & D. $t$. & 1,9 & 1,9 & 0,3 & 0,4 & 1,8 & 2,6 & 0,4 & 0,7 & 0,3 & 1,1 & 1,8 & 3,1 & 0,4 & 0,8 & 1,5 & 1,1 & 4,89 \\
\hline KR 1_21 & D. $a$. & 2,3 & 2,9 & 0,6 & 0,3 & 1,6 & 2,7 & 0,6 & 1,0 & 0,9 & 2,1 & 1,2 & 2,4 & 0,7 & 0,8 & 1,2 & 0,5 & 7,12 \\
\hline KR 1_22 & D. $i$. & 1,9 & 1,3 & 0,4 & 0,1 & 1,7 & 1,9 & 0,4 & 0,3 & 0,3 & 1,8 & 1,9 & 2,0 & 0,4 & 0,2 & 0,9 & 0,5 & 6,16 \\
\hline KR 1_23 & D. $i$. & 2,3 & 4,0 & 0,4 & 1,3 & 2,2 & 4,1 & 0,7 & 1,7 & 0,7 & 1,2 & 1,9 & 3,8 & 0,5 & 1,4 & 1,3 & 0,9 & 5,95 \\
\hline KR 1_24 & D. $i$. & 2,0 & 2,4 & 0,8 & 0,4 & 2,1 & 2,5 & 0,7 & 0,5 & 0,7 & 2,2 & 2,2 & 2,8 & 1,0 & 0,8 & 2,0 & 1,0 & 6,07 \\
\hline KR 1_25 & D. $t$. & 1,2 & 2,7 & 0,4 & 0,9 & 1,3 & 2,5 & 0,3 & 1,1 & 0,6 & 1,2 & 1,4 & 2,7 & 0,4 & 0,7 & 1,5 & 1,1 & 5,05 \\
\hline KR 1_29 & D. C. & 1,4 & 2,0 & 0,4 & 0,5 & 1,7 & 1,9 & 0,6 & 0,5 & 0,7 & 1,9 & 1,3 & 2,2 & 0,8 & 0,5 & 2,2 & 1,4 & 5,51 \\
\hline KR 1_3 & D. $i$. & 1,2 & 2,0 & 0,4 & 0,7 & 1,6 & 2,4 & 0,5 & 0,4 & 0,5 & 1,6 & 1,8 & 2,2 & 0,8 & 0,4 & 1,7 & 0,9 & 6,00 \\
\hline KR 1_31 & D. c. & 1,4 & 2,5 & 0,5 & 1,1 & 1,8 & 2,5 & 0,6 & 0,8 & 0,6 & 2,3 & 1,5 & 1,9 & 0,6 & 0,3 & 1,9 & 1,3 & 5,53 \\
\hline KR 1_4 & D. $t$. & 1,3 & 3,7 & 0,4 & 1,9 & 1,7 & 3,2 & 0,6 & 1,6 & 0,9 & 2,2 & 1,9 & 3,4 & 0,8 & 1,1 & 2,3 & 1,5 & 5,28 \\
\hline KR 1_6 & D. o. & 1,6 & 1,8 & 0,5 & 0,2 & 1,5 & 1,6 & 0,7 & 0,0 & 0,5 & 2,1 & 1,6 & 1,6 & 0,8 & 0,3 & 2,0 & 1,3 & 6,30 \\
\hline KR 1_7 & D. $i$. & 1,9 & 3,0 & 0,5 & 1,0 & 2,0 & 2,9 & 0,5 & 0,9 & 0,6 & 1,4 & 1,8 & 2,7 & 0,8 & 0,7 & 1,6 & 0,9 & 5,91 \\
\hline KR 1_8 & D. c. & 1,0 & 2,3 & 0,3 & 0,9 & 1,7 & 2,1 & 0,5 & 0,4 & 0,5 & 2,2 & 1,4 & 2,6 & 0,6 & 0,9 & 1,8 & 1,1 & 5,30 \\
\hline KR 1_9 & D. o. & 1,7 & 3,0 & 0,7 & 0,6 & 2,5 & 3,0 & 0,9 & 0,4 & 0,7 & 2,5 & 1,6 & 2,5 & 0,8 & 0,5 & 2,7 & 1,9 & 6,24 \\
\hline KR 2_1 & D. C. & 1,0 & 3,2 & 0,5 & 1,8 & 2,0 & 3,2 & 0,6 & 1,5 & 0,5 & 2,7 & 1,7 & 3,1 & 0,8 & 1,8 & 2,2 & 1,5 & 5,41 \\
\hline KR 2_3 & D. c. & 1,4 & 2,0 & 0,3 & 0,5 & 1,6 & 1,5 & 0,3 & 0,4 & 0,6 & 1,5 & 1,5 & 1,8 & 0,4 & 0,3 & 1,4 & 1,0 & 5,48 \\
\hline KR 2B_1 & D. C. & 0,9 & 1,4 & 0,2 & 0,6 & 1,4 & 1,8 & 0,5 & 0,6 & 0,4 & 1,3 & 1,5 & 1,9 & 0,5 & 0,1 & 1,5 & 0,9 & 5,52 \\
\hline KR 2B_2 & D. C. & 0,8 & 1,9 & 0,3 & 0,5 & 1,5 & 1,8 & 0,2 & 0,4 & 0,4 & 1,2 & 1,4 & 1,5 & 0,4 & 0,6 & 1,2 & 0,8 & 5,52 \\
\hline KR 2B_3 & D. C. & 1,3 & 1,5 & 0,5 & 0,6 & 1,5 & 2,1 & 0,4 & 0,9 & 0,8 & 1,9 & 1,5 & 1,9 & 0,6 & 0,2 & 2,2 & 1,5 & 5,58 \\
\hline KR 3_1 & D. $i$. & 1,7 & 1,8 & 0,5 & 0,4 & 1,7 & 1,9 & 0,7 & 0,6 & 0,5 & 2,1 & 1,8 & 1,7 & 0,7 & $-0,1$ & 2,1 & 1,4 & 6,31 \\
\hline KR 3_2 & D. $i$. & 1,4 & 2,3 & 0,6 & 0,4 & 1,9 & 2,4 & 0,6 & 0,4 & 0,8 & 2,1 & 2,0 & 2,2 & 0,7 & 0,5 & 1,9 & 1,2 & 6,12 \\
\hline KR 4_1 & D. $t$. & 1,4 & 1,8 & 0,3 & 0,9 & 1,1 & 1,9 & 0,3 & 0,6 & 0,3 & 0,9 & 1,3 & 2,1 & 0,4 & 0,4 & 0,9 & 0,5 & 4,98 \\
\hline KR 4_2 & D. $a$. & 1,9 & 1,5 & 0,8 & $-0,4$ & 1,8 & 1,5 & 0,5 & 0,2 & 0,6 & 2,2 & 2,1 & 1,4 & 0,7 & 0,4 & 1,1 & 0,4 & 7,18 \\
\hline KR 4_3 & D. $a$. & 1,7 & 1,6 & 0,5 & $-0,2$ & 1,8 & 1,7 & 0,5 & $-0,1$ & 0,5 & 1,8 & 1,5 & 1,6 & 0,5 & $-0,1$ & 1,6 & 1,1 & 7,17 \\
\hline KRK 1_1 & D. $i$. & 2,0 & 3,1 & 0,6 & 0,6 & 2,0 & 2,8 & 0,7 & 0,7 & 0,5 & 1,8 & 1,8 & 3,1 & 0,7 & 0,9 & 2,0 & 1,3 & 5,94 \\
\hline KRK 1_2 & D. $t$. & 1,5 & 2,0 & 0,6 & 0,7 & 1,6 & 1,7 & 0,6 & 0,2 & 0,4 & 1,0 & 1,3 & 2,1 & 0,6 & 0,9 & 1,4 & 0,7 & 4,99 \\
\hline KRK 1_3 & D. $i$. & 2,2 & 2,8 & 0,8 & 0,6 & 2,1 & 2,7 & 0,6 & 0,5 & 0,6 & 2,0 & 1,9 & 2,5 & 0,7 & 0,7 & 0,8 & 0,1 & 6,07 \\
\hline KRK 3 & D. $a$. & 2,1 & 2,5 & 0,8 & 0,3 & 2,1 & 2,1 & 0,5 & 0,4 & 0,4 & 1,5 & 2,0 & 2,5 & 0,7 & 0,7 & 2,0 & 1,2 & 7,08 \\
\hline KTI 01 & D. c. & 0,5 & 2,6 & 0,3 & 1,7 & 1,9 & 2,8 & 0,9 & 1,6 & 0,8 & 3,5 & 1,6 & 2,6 & 0,4 & 1,5 & 2,2 & 2,2 & 5,39 \\
\hline KTI 02 & D. c. & 1,1 & 3,2 & 0,2 & 2,1 & 1,6 & 3,6 & 1,1 & 2,0 & 0,8 & 4,1 & 1,1 & 3,1 & 0,5 & 1,5 & 1,7 & 1,8 & 5,24 \\
\hline KTI 03 & D. C. & 1,5 & 3,1 & 0,4 & 1,3 & 1,8 & 3,7 & 0,9 & 1,8 & 1,0 & 2,2 & 1,4 & 2,2 & 0,5 & 0,9 & 1,8 & 1,9 & 5,28 \\
\hline KUB 01 & D. t. & 1,5 & 2,0 & 0,5 & 0,6 & 1,6 & 1,9 & 0,4 & 0,7 & 0,3 & 1,3 & 1,4 & 1,9 & 0,7 & 0,7 & 1,4 & 1,5 & 4,89 \\
\hline KUB 02 & D. $z$. & 2,1 & 2,8 & 0,4 & 0,7 & 2,0 & 2,7 & 0,6 & 1,0 & 0,7 & 1,3 & 1,1 & 3,4 & 0,6 & 0,9 & 1,6 & 1,7 & 5,16 \\
\hline KUB 03 & D. $a$. & 1,6 & 1,7 & 0,5 & $-0,2$ & 2,0 & 1,8 & 0,7 & 0,1 & 0,8 & 2,1 & 2,0 & 1,6 & 0,8 & $-0,3$ & 2,3 & 2,4 & 7,19 \\
\hline KUB 04 & D. $a$. & 2,3 & 3,1 & 0,6 & 0,7 & 2,0 & 3,1 & 0,6 & 1,1 & 0,6 & 2,0 & 1,8 & 3,0 & 0,6 & 0,8 & 1,4 & 1,4 & 7,33 \\
\hline KUB 05 & D. $i$. & 1,6 & 2,6 & 0,4 & 0,7 & 1,6 & 2,4 & 0,8 & 0,4 & 0,6 & 1,9 & 1,5 & 2,1 & 0,6 & 0,7 & 1,9 & 2,0 & 6,42 \\
\hline KUB 06 & D. $a$. & 2,5 & 3,2 & 0,6 & 0,5 & 2,7 & 3,0 & 0,8 & 0,7 & 0,7 & 2,8 & 2,0 & 3,0 & 0,8 & 1,3 & 1,9 & 2,0 & 7,16 \\
\hline KUB 07 & D. o. & 1,8 & 2,5 & 0,8 & 0,9 & 2,0 & 2,6 & 0,9 & 0,7 & 0,7 & 2,5 & 1,8 & 2,6 & 0,8 & 1,0 & 2,6 & 2,6 & 6,37 \\
\hline KUB 08_1 & D. z. & 1,1 & 2,1 & 0,3 & 0,9 & 1,3 & 1,9 & 0,7 & 0,4 & 0,5 & 1,5 & 1,1 & 2,0 & 0,5 & 0,9 & 1,8 & 1,9 & 4,94 \\
\hline KUB 08_2 & D. $t$. & 1,9 & 2,0 & 0,4 & 0,4 & 1,4 & 1,8 & 0,5 & 0,1 & 0,4 & 0,8 & 1,6 & 2,2 & 0,5 & 0,2 & 1,3 & 1,3 & 5,40 \\
\hline KUB 08_3 & D. C. & 1,9 & 2,3 & 0,4 & 0,4 & 1,9 & 2,2 & 0,5 & 0,5 & 0,4 & 2,0 & 1,9 & 1,9 & 0,4 & 0,3 & 1,9 & 2,0 & 5,48 \\
\hline
\end{tabular}




\begin{tabular}{|c|c|c|c|c|c|c|c|c|c|c|c|c|c|c|c|c|c|c|}
\hline KUB 09 & D. c. & 1,9 & 2,2 & 0,4 & 2,4 & 2,0 & 2,4 & 0,5 & 0,7 & 0,7 & 2,0 & 1,8 & 3,1 & 0,5 & 0,6 & 1,5 & 1,6 & 5,48 \\
\hline KUB 10 & D. $a$. & 2,6 & 3,5 & 0,8 & 1,3 & 3,0 & 3,7 & 1,0 & 1,5 & 1,3 & 3,2 & 2,1 & 4,3 & 1,0 & 2,1 & 2,0 & 2,2 & 7,14 \\
\hline KUB 11 & D. $a$. & 1,9 & 2,2 & 0,8 & 0,1 & 1,9 & 2,0 & 0,8 & 0,3 & 0,9 & 2,3 & 2,0 & 2,1 & 0,8 & 0,0 & 1,4 & 1,4 & 7,23 \\
\hline KUB 12 & D. $a$. & 1,5 & 1,5 & 0,7 & $-0,3$ & 1,8 & 1,6 & 0,5 & $-0,3$ & 0,8 & 2,0 & 2,0 & 1,7 & 0,8 & $-0,4$ & 1,4 & 1,5 & 7,27 \\
\hline KUB 13 & D. o. & 1,8 & 2,3 & 0,5 & 1,3 & 2,0 & 2,2 & 0,8 & 0,4 & 0,7 & 2,7 & 2,0 & 1,9 & 0,6 & $-0,3$ & 1,9 & 1,9 & 6,33 \\
\hline KUB 14A & D. o. & 2,0 & 2,7 & 0,4 & 0,8 & 1,9 & 2,5 & 0,7 & 0,6 & 0,7 & 1,8 & 1,8 & 2,1 & 0,5 & 0,4 & 1,6 & 1,7 & 6,30 \\
\hline KUB 14B & D. o. & 1,7 & 2,0 & 0,5 & 0,2 & 1,7 & 1,9 & 0,6 & 0,1 & 0,6 & 2,1 & 1,4 & 1,9 & 0,7 & 0,4 & 2,0 & 2,1 & 6,29 \\
\hline KUB 15 & D. z. & 1,3 & 1,9 & 0,3 & 0,5 & 1,5 & 1,9 & 0,5 & 0,4 & 0,5 & 0,6 & 1,4 & 1,8 & 0,6 & 0,5 & 1,3 & 1,4 & 5,29 \\
\hline KUB $16 \mathrm{~A}$ & D. i. & 1,6 & 2,4 & 0,4 & 0,6 & 1,8 & 2,5 & 0,9 & 0,6 & 0,6 & 1,5 & 2,0 & 3,0 & 0,8 & 1,0 & 2,4 & 2,6 & 6,42 \\
\hline KVIL1 & D. $a$. & 1,5 & 1,3 & 0,6 & $-0,2$ & 1,7 & 1,2 & 0,7 & $-0,3$ & 0,6 & 2,0 & 1,5 & 1,4 & 0,6 & $-0,3$ & 2,0 & 1,4 & 7,45 \\
\hline LAK 01 & D. $a$. & 2,0 & 1,9 & 0,9 & 0,2 & 2,0 & 2,2 & 0,6 & 0,1 & 0,5 & 2,3 & 2,2 & 1,7 & 0,9 & $-0,6$ & 1,0 & 1,0 & 7,19 \\
\hline LAK 02 & D. $a$. & 2,4 & 2,4 & 0,8 & 0,1 & 2,4 & 2,6 & 0,7 & $-0,2$ & 0,5 & 2,2 & 2,3 & 2,1 & 0,8 & 0,3 & 0,7 & 0,7 & 7,28 \\
\hline LAK 03 & D. $a$. & 2,8 & 3,7 & 0,6 & 1,0 & 2,5 & 3,9 & 1,0 & 1,7 & 0,6 & 3,1 & 3,1 & 3,8 & 0,8 & 1,4 & 0,9 & 1,1 & 6,90 \\
\hline LAK 04 & D. $i$. & 1,7 & 3,4 & 0,5 & 1,8 & 2,0 & 3,4 & 1,0 & 1,4 & 0,7 & 2,4 & 2,1 & 3,8 & 0,9 & 1,3 & 1,9 & 2,2 & 6,37 \\
\hline LAK 05 & D. i. & 1,5 & 4,9 & 0,4 & 3,2 & 2,5 & 4,6 & 1,0 & 3,0 & 0,7 & 3,7 & 1,7 & 4,9 & 0,6 & 2,8 & 1,0 & 1,1 & 6,36 \\
\hline LAK 06 & D. $a$. & 2,2 & 2,9 & 0,9 & 0,5 & 2,6 & 2,7 & 0,7 & 0,2 & 0,7 & 2,6 & 2,5 & 2,9 & 0,9 & 0,6 & 1,5 & 1,5 & 7,09 \\
\hline MH1 & D. o. & 1,7 & 1,9 & 0,4 & 0,3 & 1,6 & 2,0 & 0,6 & 0,3 & 0,7 & 2,1 & 1,4 & 1,8 & 0,6 & 0,5 & 2,0 & 2,1 & 6,42 \\
\hline MH10 & D. o. & 1,5 & 0,6 & 0,4 & 0,8 & 1,6 & 1,9 & 0,6 & 0,5 & 0,7 & 2,4 & 1,7 & 2,1 & 0,6 & 0,8 & 2,0 & 2,1 & 6,39 \\
\hline $\mathrm{MH} 2$ & D.t. & 1,0 & 2,0 & 0,3 & 1,0 & 1,1 & 1,9 & 0,5 & 0,8 & 0,4 & 1,2 & 1,2 & 2,3 & 0,5 & 0,9 & 1,7 & 1,8 & 5,50 \\
\hline MH3 & D. o. & 2,2 & 3,1 & 0,6 & 1,0 & 2,6 & 3,4 & 0,9 & 1,0 & 0,6 & 2,5 & 1,9 & 3,6 & 0,8 & 1,8 & 2,3 & 2,5 & 6,51 \\
\hline MH4 & D. o. & 2,2 & 2,7 & 0,4 & 0,6 & 1,4 & 2,3 & 0,6 & 0,6 & 0,6 & 2,2 & 1,9 & 2,8 & 0,9 & 1,0 & 2,0 & 2,2 & 6,43 \\
\hline MH5 & D. $a$. & 2,1 & 3,2 & 0,6 & 0,9 & 2,2 & 3,3 & 0,8 & 1,2 & 0,9 & 2,8 & 1,8 & 2,5 & 0,8 & 0,7 & 1,6 & 1,7 & 7,32 \\
\hline MH6 & D. o. & 1,5 & 2,4 & 0,5 & 0,9 & 2,1 & 2,2 & 0,7 & 0,5 & 0,6 & 2,1 & 1,5 & 2,5 & 0,8 & 0,5 & 2,4 & 2,5 & 6,49 \\
\hline MH7 & D. o. & 1,2 & 2,5 & 0,5 & 0,8 & 1,9 & 2,7 & 0,8 & 0,5 & 0,7 & 2,4 & 1,6 & 2,8 & 0,7 & 0,9 & 2,2 & 2,3 & 6,37 \\
\hline MH8 & D. o. & 1,7 & 1,9 & 0,5 & 0,7 & 1,5 & 2,1 & 0,7 & 0,6 & 0,6 & 2,5 & 1,5 & 2,2 & 0,7 & 0,8 & 1,9 & 2,1 & 6,46 \\
\hline MH9 & D. o. & 1,4 & 1,8 & 0,5 & 0,1 & 1,9 & 1,9 & 0,6 & 0,3 & 0,5 & 2,5 & 1,4 & 1,9 & 0,9 & 0,2 & 2,2 & 2,4 & 6,36 \\
\hline OLS 1 & D. $a$. & 2,8 & 4,4 & 0,9 & 1,2 & 2,5 & 3,8 & 1,0 & 1,4 & 0,9 & 3,7 & 2,5 & 4,2 & 0,8 & 1,9 & 1,2 & 1,1 & 7,32 \\
\hline OLS 10 & D. $a$. & 2,5 & 2,4 & 0,5 & 0,0 & 2,4 & 2,1 & 0,9 & $-0,3$ & 0,8 & 3,2 & 2,1 & 2,3 & 0,9 & $-0,2$ & 1,6 & 1,8 & 7,31 \\
\hline OLS 11 & D. o. & 1,3 & 2,3 & 0,5 & 1,1 & 1,7 & 2,4 & 0,9 & 0,7 & 0,5 & 2,5 & 1,9 & 2,3 & 0,9 & 0,5 & 2,3 & 2,5 & 6,34 \\
\hline OLS 12 & D. i. & 2,2 & 3,5 & 0,7 & 1,2 & 2,0 & 3,1 & 1,2 & 1,2 & 0,7 & 2,6 & 2,8 & 2,9 & 0,8 & 0,8 & 2,6 & 2,7 & 6,44 \\
\hline OLS 13 & D. o. & 1,5 & 2,4 & 0,5 & 0,6 & 1,7 & 2,4 & 0,9 & 1,1 & 0,7 & 2,4 & 1,9 & 2,4 & 0,7 & 0,7 & 2,6 & 2,7 & 6,38 \\
\hline OLS 14 & D. $a$. & 2,1 & 2,5 & 0,8 & 0,0 & 2,3 & 2,2 & 0,7 & $-0,1$ & 0,7 & 3,4 & 2,4 & 2,5 & 0,9 & $-0,2$ & 1,3 & 1,3 & 7,32 \\
\hline OLS 15 & D. $a$. & 1,6 & 1,9 & 0,7 & $-0,2$ & 2,6 & 2,1 & 0,5 & $-0,4$ & 0,7 & 3,0 & 2,1 & 2,0 & 0,8 & $-0,3$ & 2,0 & 2,1 & 7,41 \\
\hline OLS 16 & D. $a$. & 2,1 & 1,7 & 0,7 & $-0,1$ & 1,8 & 1,7 & 0,4 & $-0,2$ & 0,8 & 2,2 & 2,0 & 1,7 & 0,8 & $-0,3$ & 2,1 & 2,2 & 7,35 \\
\hline OLS 17 & D. $a$. & 1,9 & 2,0 & 0,7 & 1,5 & 2,2 & 2,1 & 0,9 & $-0,2$ & 0,9 & 2,3 & 2,0 & 2,1 & 0,9 & $-0,3$ & 2,4 & 2,5 & 7,40 \\
\hline OLS 18 & D. $a$. & 2,2 & 2,8 & 0,7 & 0,4 & 1,9 & 2,6 & 0,7 & 0,7 & 0,8 & 2,1 & 1,9 & 2,3 & 0,7 & 1,4 & 1,7 & 1,7 & 7,35 \\
\hline OLS 19 & D. $a$. & 2,5 & 2,5 & 0,8 & 0,1 & 2,0 & 2,2 & 0,8 & 0,5 & 0,8 & 2,9 & 1,8 & 2,6 & 0,8 & 0,6 & 1,6 & 1,6 & 7,39 \\
\hline OLS 2 & D. $a$. & 2,2 & 2,6 & 0,6 & 0,3 & 2,3 & 2,3 & 1,0 & 0,2 & 0,7 & 2,6 & 1,6 & 2,8 & 0,7 & 0,8 & 1,3 & 1,4 & 7,39 \\
\hline OLS 3 & D. $a$. & 2,2 & 2,7 & 0,6 & 0,3 & 2,2 & 2,8 & 0,8 & 0,2 & 0,8 & 2,7 & 1,8 & 2,8 & 0,8 & 0,3 & 1,4 & 1,5 & 7,20 \\
\hline OLS 4 & D. $a$. & 2,3 & 2,8 & 0,7 & 0,5 & 2,3 & 2,2 & 0,9 & 0,4 & 0,5 & 2,3 & 2,2 & 2,7 & 0,7 & 0,6 & 1,6 & 1,6 & 6,84 \\
\hline OLS 5 & D. $a$. & 2,0 & 2,4 & 0,6 & 0,1 & 2,6 & 2,5 & 0,8 & 0,1 & 0,9 & 2,7 & 1,9 & 2,1 & 0,8 & $-0,1$ & 1,5 & 1,6 & 7,32 \\
\hline OLS 6 & D. $a$. & 2,5 & 2,4 & 0,7 & 0,0 & 2,3 & 2,2 & 0,8 & 0,1 & 0,9 & 2,9 & 2,1 & 2,2 & 0,9 & $-0,2$ & 2,0 & 2,1 & 7,28 \\
\hline OLS 7 & D. $a$. & 2,3 & 2,8 & 0,8 & 0,4 & 2,3 & 2,8 & 0,9 & 0,6 & 0,7 & 2,9 & 2,2 & 2,6 & 0,8 & 0,6 & 1,1 & 1,1 & 7,11 \\
\hline OLS 8 & D. $a$. & 2,1 & 2,1 & 0,6 & $-0,1$ & 2,3 & 2,3 & 0,7 & 0,3 & 0,7 & 2,8 & 2,0 & 2,2 & 0,8 & 0,3 & 1,3 & 1,4 & 7,25 \\
\hline OLS 9 & D. $a$. & 1,6 & 1,6 & 0,7 & 0,0 & 2,0 & 2,0 & 0,6 & 0,0 & 0,7 & 2,7 & 1,9 & 2,0 & 0,6 & 0,0 & 1,1 & 1,0 & 7,25 \\
\hline OST 02 & D. z. & 1,1 & 2,6 & 0,4 & 1,6 & 1,5 & 2,6 & 0,6 & 1,1 & 0,5 & 1,4 & 1,2 & 2,7 & 0,5 & 1,6 & 1,8 & 1,8 & 5,28 \\
\hline OST 03 & D. z. & 1,5 & 2,0 & 0,5 & 0,6 & 1,6 & 2,1 & 0,6 & 0,4 & 0,4 & 1,3 & 1,6 & 2,4 & 0,9 & 0,4 & 1,6 & 1,7 & 5,28 \\
\hline OST 04 & D. z. & 1,4 & 3,2 & 0,6 & 1,7 & 1,4 & 2,8 & 0,7 & 1,3 & 0,5 & 1,5 & 1,5 & 2,9 & 0,9 & 1,8 & 1,8 & , & 5,32 \\
\hline OST 05 & D. z. & 1,5 & 2,8 & 0,4 & 1,4 & 1,6 & 2,7 & 0,7 & 1,1 & 0,6 & 0,9 & 1,5 & 2,5 & 0,9 & 1,4 & 1,7 & 1,9 & 5,30 \\
\hline OST 06 & D. z. & 1,6 & 2,9 & 0,4 & 1,0 & 2,2 & 2,6 & 0,7 & 0,9 & 0,4 & 1,2 & 1,9 & 2,2 & 0,7 & 0,7 & 1,8 & 2,0 & 5,20 \\
\hline
\end{tabular}




\begin{tabular}{|c|c|c|c|c|c|c|c|c|c|c|c|c|c|c|c|c|c|c|}
\hline POL 1 & D. $t$. & 1,6 & 2,8 & 0,5 & 1,0 & 1,6 & 3,1 & 0,5 & 1,0 & 0,3 & 1,2 & 1,5 & 3,2 & 0,8 & 1,5 & 1,5 & 1,7 & 5,06 \\
\hline POL 2 & D. $t$. & 1,7 & 2,2 & 0,5 & 0,5 & 1,6 & 2,4 & 0,5 & 0,6 & 0,3 & 1,4 & 1,5 & 2,4 & 0,8 & 0,4 & 1,5 & 1,6 & 4,98 \\
\hline POL 4 & D.t. & 1,5 & 1,9 & 0,5 & 0,4 & 1,5 & 1,8 & 0,5 & 0,1 & 0,4 & 1,4 & 1,3 & 2,1 & 0,8 & 0,6 & 1,5 & 1,6 & 4,99 \\
\hline POL 5 & D.t. & 1,6 & 3,4 & 0,6 & 1,2 & 1,8 & 3,4 & 0,7 & 0,9 & 0,4 & 1,5 & 1,7 & 3,4 & 0,8 & 1,2 & 1,8 & 1,9 & 4,92 \\
\hline POL 6 & D.t. & 1,8 & 2,6 & 0,5 & 0,8 & 1,8 & 2,5 & 0,5 & 0,8 & 0,4 & 1,5 & 1,3 & 2,6 & 0,7 & 1,1 & 1,5 & 1,6 & 4,97 \\
\hline POL 7 & D. z. & 1,9 & 4,4 & 0,5 & 2,8 & 2,2 & 4,6 & 0,6 & 2,5 & 0,6 & 1,6 & 1,1 & 3,3 & 0,5 & 1,4 & 1,6 & 1,6 & 5,22 \\
\hline POL 8 & D.t. & 1,5 & 2,4 & 0,6 & 0,8 & 1,3 & 2,3 & 0,5 & 1,1 & 0,3 & 1,0 & 1,6 & 2,3 & 0,8 & 0,7 & 1,4 & 1,5 & 4,97 \\
\hline POL 9 & D.t. & 1,7 & 2,5 & 0,4 & 0,6 & 1,6 & 2,2 & 0,4 & 0,5 & 0,4 & 1,3 & 1,5 & 2,3 & 0,6 & 0,7 & 1,3 & 1,4 & 4,98 \\
\hline POL3 & D.t. & 1,7 & 3,2 & 0,4 & 1,4 & 1,8 & 3,2 & 0,5 & 1,3 & 0,4 & 0,9 & 1,5 & 2,8 & 0,6 & 1,0 & 1,4 & 1,5 & 5,07 \\
\hline PRI 01 & D. C. & 0,6 & 2,4 & 0,3 & 1,6 & 1,5 & 2,2 & 0,8 & 1,5 & 0,8 & 2,9 & 1,0 & 2,4 & 0,5 & 1,4 & 2,0 & 2,1 & 5,52 \\
\hline PRI 02 & D. C. & 1,0 & 3,1 & 0,4 & 2,5 & 1,5 & 2,9 & 0,9 & 1,7 & 0,9 & 3,3 & 1,1 & 2,5 & 0,7 & 1,8 & 2,3 & 2,5 & 5,59 \\
\hline PRI 03 & D. C. & 0,6 & 3,0 & 0,3 & 2,0 & 1,9 & 2,6 & 0,7 & 1,8 & 0,6 & 3,5 & 1,0 & 2,6 & 0,3 & 1,4 & 1,6 & 1,7 & 5,56 \\
\hline PRI 04 & D.c. & 1,1 & 4,5 & 0,4 & 3,3 & 1,7 & 4,0 & 0,8 & 2,5 & 1,2 & 3,5 & 1,6 & 3,5 & 0,3 & 2,2 & 2,7 & 2,7 & 5,47 \\
\hline PRI 05 & D. c. & 1,0 & 3,1 & 0,3 & 2,2 & 2,0 & 2,9 & 0,6 & 1,5 & 0,8 & 3,4 & 1,4 & 3,4 & 0,4 & 1,8 & 2,0 & 2,1 & 5,46 \\
\hline PRIL 01 & D. $a$. & 2,4 & 2,5 & 0,6 & 0,2 & 2,0 & 1,9 & 0,8 & 0,0 & 0,5 & 2,4 & 2,1 & 2,9 & 0,8 & 0,8 & 1,5 & 1,6 & 7,21 \\
\hline PRIL 02 & D. $a$. & 2,7 & 3,8 & 1,0 & 1,4 & 2,7 & 3,8 & 0,8 & 1,3 & 0,8 & 2,9 & 2,3 & 4,0 & 0,8 & 2,3 & 1,8 & 1,7 & 7,20 \\
\hline PRIL 03 & D. C. & 0,8 & 3,0 & 0,3 & 2,0 & 1,8 & 3,4 & 0,8 & 1,9 & 0,8 & 2,6 & 1,2 & 3,1 & 0,6 & 1,9 & 2,0 & 2,2 & 5,44 \\
\hline PRIL 04 & D. $a$. & 2,1 & 3,3 & 0,6 & 1,1 & 2,5 & 3,4 & 0,8 & 1,1 & 0,8 & 3,5 & 2,3 & 3,5 & 0,7 & 1,0 & 1,8 & 1,9 & 7,25 \\
\hline PRIL 05 & D. $a$. & 2,5 & 2,7 & 0,7 & 0,4 & 2,5 & 2,3 & 0,7 & $-0,2$ & 0,8 & 2,6 & 2,0 & 2,4 & 0,7 & 0,4 & 1,6 & 1,6 & 7,27 \\
\hline PRIL 06 & D. $i$. & 1,6 & 3,5 & 0,5 & 1,7 & 2,0 & 3,4 & 1,0 & 1,2 & 0,7 & 2,8 & 1,8 & 3,5 & 0,6 & 1,3 & 2,3 & 2,3 & 6,23 \\
\hline PRIL 07 & D. c. & 1,1 & 2,7 & 0,3 & 1,9 & 1,9 & 3,1 & 0,7 & 1,3 & 0,9 & 3,0 & 1,6 & 3,1 & 0,6 & 1,7 & 2,0 & 2,2 & 5,61 \\
\hline PRIL1 & D. $a$. & 2,6 & 3,9 & 0,9 & 1,4 & 2,7 & 4,3 & 0,9 & 1,5 & 0,8 & 2,5 & 2,2 & 3,9 & 0,9 & 1,5 & 2,7 & 1,8 & 7,22 \\
\hline PRIL2 & D. c. & 0,9 & 3,5 & 0,4 & 2,6 & 1,7 & 3,7 & 1,0 & 2,7 & 0,7 & 3,6 & 1,1 & 3,7 & 0,5 & 2,4 & 2,7 & 2,2 & 5,54 \\
\hline PRIL3 & D. C. & 1,5 & 3,3 & 0,4 & 2,0 & 2,3 & 2,9 & 0,9 & 1,6 & 0,8 & 3,1 & 1,2 & 2,8 & 0,5 & 1,4 & 2,5 & 2,0 & 5,53 \\
\hline PRIL4 & D. c. & 0,9 & 2,1 & 0,5 & 1,3 & 1,4 & 2,4 & 0,6 & 1,0 & 0,7 & 1,9 & 1,4 & 1,9 & 0,5 & 0,6 & 2,1 & 1,6 & 5,56 \\
\hline R1 & D. c. & 1,3 & 2,3 & 0,3 & 0,7 & 1,9 & 2,1 & 0,6 & 0,3 & 0,8 & 2,2 & 1,4 & 2,4 & 0,3 & 0,6 & 1,8 & 1,5 & 5,51 \\
\hline R10 & D. $i$. & 2,0 & 2,6 & 0,7 & 0,5 & 2,0 & 2,4 & 0,7 & 0,5 & 0,6 & 2,0 & 1,5 & 2,3 & 0,9 & 0,6 & 2,2 & 1,3 & 6,41 \\
\hline R11 & D. C. & 0,7 & 1,8 & 0,3 & 1,3 & 1,5 & 1,8 & 0,6 & 0,7 & 0,4 & 2,0 & 1,4 & 2,2 & 0,5 & 1,1 & 2,0 & 1,5 & 5,51 \\
\hline R12 & D. c. & 1,2 & 2,2 & 0,3 & 0,6 & 1,7 & 2,3 & 0,4 & 0,6 & 0,8 & 2,1 & 1,5 & 2,8 & 0,7 & 0,5 & 1,8 & 1,1 & 5,47 \\
\hline R13 & D.t. & 1,3 & 1,7 & 0,5 & 0,4 & 1,4 & 1,6 & 0,5 & 0,4 & 0,3 & 1,5 & 1,0 & 1,3 & 0,6 & 0,4 & 1,5 & 0,8 & 5,22 \\
\hline R14 & D. $t$. & 1,1 & 1,5 & 0,3 & 0,4 & 1,2 & 1,4 & 0,4 & 0,1 & 0,4 & 1,0 & 1,2 & 1,7 & 0,4 & 0,3 & 1,3 & 0,8 & 5,21 \\
\hline R15 & D.c. & 0,9 & 2,4 & 0,3 & 1,4 & 1,6 & 2,5 & 0,8 & 0,8 & 0,6 & 2,3 & 1,1 & 2,1 & 0,5 & 0,9 & 1,9 & 1,4 & 5,52 \\
\hline R17 & D. c. & 1,4 & 1,5 & 0,5 & 0,5 & 1,3 & 1,5 & 0,6 & 0,4 & 0,5 & 1,7 & 1,3 & 1,6 & 0,4 & 0,4 & 2,3 & 1,9 & 5,00 \\
\hline R18 & D.t. & 1,2 & 1,6 & 0,4 & 0,4 & 1,2 & 1,4 & 0,3 & 0,3 & 0,2 & 1,2 & 1,1 & 1,5 & 0,7 & 0,2 & 1,3 & 0,6 & 5,10 \\
\hline R19 & D. $i$. & 1,9 & 2,1 & 0,3 & 0,3 & 2,0 & 1,6 & 0,9 & 0,2 & 0,3 & 1,2 & 1,3 & 1,5 & 0,5 & 0,4 & 2,1 & 1,6 & 6,37 \\
\hline R2 & D. C. & 1,7 & 2,4 & 0,7 & 2,5 & 1,6 & 2,3 & 0,6 & 0,6 & 0,4 & 1,8 & 1,6 & 2,3 & 0,7 & 0,9 & 1,7 & 1,1 & 5,29 \\
\hline R20 & D.c. & 1,7 & 2,7 & 0,5 & 1,0 & 1,8 & 2,4 & 0,5 & 0,7 & 0,5 & 1,6 & 1,7 & 2,7 & 0,9 & 0,6 & 1,8 & 0,9 & 5,43 \\
\hline R21 & D.t. & 1,1 & 2,2 & 0,5 & 1,0 & 1,5 & 2,6 & 0,5 & 0,6 & 0,5 & 1,8 & 1,4 & 2,2 & 0,7 & 0,6 & 1,5 & 0,8 & 4,95 \\
\hline R23 & D. $i$. & 1,5 & 3,0 & 0,5 & 1,5 & 1,6 & 3,4 & 0,6 & 1,6 & 0,9 & 2,5 & 1,4 & 3,5 & 0,9 & 1,8 & 1,7 & 0,9 & 6,20 \\
\hline R24 & D.c. & 1,1 & 1,9 & 0,3 & 0,6 & 1,3 & 1,4 & 0,5 & 0,2 & 0,5 & 1,6 & 1,2 & 1,7 & 0,4 & 0,5 & 1,7 & 1,3 & 5,37 \\
\hline R26 & D. $a$. & 1,5 & 1,3 & 0,7 & $-0,4$ & 1,4 & 1,1 & 0,3 & $-0,4$ & 0,7 & 1,7 & 1,0 & 1,1 & 0,7 & 0,2 & 1,8 & 1,2 & 7,31 \\
\hline R27 & D.z. & 1,6 & 3,0 & 0,5 & 1,8 & 1,7 & 3,7 & 0,7 & 2,1 & 0,6 & 2,0 & 1,4 & 2,9 & 0,7 & 1,9 & 1,9 & 1,3 & 5,10 \\
\hline R28 & $v$ & 0,6 & 2,4 & 0,3 & 1,4 & 1,5 & 2,2 & 0,6 & 1,2 & 0,5 & 2,5 & 0,9 & 1,9 & 0,4 & 0,7 & 1,5 & 1,0 & 5,01 \\
\hline R29 & D.c. & 1,4 & 2,3 & 0,3 & 1,1 & 1,7 & 2,0 & 0,4 & 0,4 & 0,8 & 2,1 & 1,5 & 1,6 & 0,3 & 0,8 & 1,7 & 1,4 & 5,54 \\
\hline R3 & D.c. & 0,8 & 1,6 & 0,4 & 0,4 & 1,4 & 1,2 & 0,6 & 0,5 & 0,3 & 1,9 & 1,4 & 1,9 & 0,5 & 0,3 & 1,8 & 1,3 & 5,48 \\
\hline R30 & D.c. & 1,1 & 2,8 & 0,5 & 2,1 & 1,6 & 2,4 & 0,8 & 1,0 & 0,6 & 2,1 & 1,5 & 2,7 & 0,3 & 1,2 & 1,9 & 1,5 & 5,53 \\
\hline R31 & & 2,1 & 3,0 & 0,6 & 0,4 & 2,4 & 2,6 & 0,8 & 0,7 & 0,5 & 2,8 & 1,8 & 2,4 & 0,8 & 1,0 & 1,5 & 0,7 & 7,24 \\
\hline R32 & D. c. & 0,5 & 2,1 & 0,4 & 1,9 & 1,3 & 1,8 & 0,5 & 0,7 & 0,5 & 1,8 & 1,3 & 2,0 & 0,4 & 0,5 & 1,8 & 1,3 & 5,41 \\
\hline R33 & D. c. & 1,3 & 2,6 & 0,4 & 1,3 & 1,7 & 1,9 & 0,5 & 1,7 & 0,5 & 1,9 & 1,6 & 2,1 & 1,8 & 0,3 & 1,7 & $-0,1$ & 5,44 \\
\hline R34 & D. C. & 0,9 & 1,9 & 0,3 & 0,9 & 1,4 & 1,9 & 0,5 & 0,5 & 0,6 & 2,2 & 1,5 & 1,8 & 0,6 & 0,7 & 1,5 & 0,9 & 5,01 \\
\hline
\end{tabular}




\begin{tabular}{|c|c|c|c|c|c|c|c|c|c|c|c|c|c|c|c|c|c|c|}
\hline R35 & D. o. & 1,3 & 2,8 & 0,3 & 1,6 & 1,7 & 2,9 & 0,9 & 1,3 & 0,5 & 2,4 & 1,3 & 2,9 & 0,6 & 1,0 & 0,9 & 0,3 & 6,30 \\
\hline R36 & D. z. & 1,1 & 1,8 & 0,5 & 0,6 & 1,3 & 1,9 & 0,5 & 0,6 & 0,3 & 1,2 & 0,7 & 1,7 & 0,6 & 0,8 & 1,4 & 0,8 & 5,44 \\
\hline R37 & D. $t$. & 1,4 & 0,7 & 0,4 & 0,8 & 1,1 & 2,1 & 0,4 & 0,8 & 0,4 & 1,3 & 1,0 & 1,8 & 0,7 & 0,6 & 1,5 & 0,8 & 5,00 \\
\hline R38 & D. z. & 1,1 & 1,7 & 0,4 & 0,6 & 1,3 & 1,5 & 0,4 & 0,4 & 0,3 & 1,3 & 1,2 & 1,2 & 0,6 & 0,5 & 1,4 & 0,8 & 5,28 \\
\hline R39 & D. $i$. & 1,6 & 1,7 & 0,6 & 0,3 & 1,7 & 1,5 & 0,7 & 0,0 & 0,5 & 2,0 & 1,5 & 1,6 & 0,7 & 0,3 & 1,9 & 1,2 & 6,43 \\
\hline R4 & D. C. & 1,3 & 2,4 & 0,5 & 0,9 & 1,4 & 2,3 & 0,7 & 0,7 & 0,5 & 1,7 & 1,4 & 2,0 & 0,7 & 0,9 & 1,6 & 1,0 & 5,37 \\
\hline R40 & D. c. & 1,3 & 2,7 & 0,4 & 1,6 & 2,1 & 2,3 & 0,7 & 0,8 & 0,9 & 2,7 & 1,4 & 2,8 & 0,7 & 0,6 & 2,5 & 1,8 & 5,58 \\
\hline R41 & D. $a$. & 1,9 & 2,3 & 0,6 & 0,6 & 2,0 & 2,2 & 0,9 & 0,4 & 0,6 & 2,5 & 1,2 & 2,0 & 0,8 & 0,4 & 2,1 & 1,3 & 7,39 \\
\hline R42 & D. $t$. & 1,3 & 1,3 & 0,3 & 0,3 & 1,2 & 1,4 & 0,3 & 0,4 & 0,4 & 1,3 & 0,7 & 1,7 & 0,7 & 0,9 & 1,3 & 0,6 & 5,01 \\
\hline R43 & D. z. & 1,1 & 1,6 & 0,3 & 0,4 & 1,3 & 1,6 & 0,4 & 0,5 & 0,4 & 1,3 & 1,3 & 1,5 & 0,3 & 0,5 & 1,2 & 0,9 & 5,36 \\
\hline R44 & D. C. & 0,8 & 1,5 & 0,3 & 0,7 & 1,3 & 1,7 & 0,7 & 0,3 & 0,4 & 1,6 & 1,1 & 1,6 & 0,4 & 0,4 & 1,8 & 1,3 & 5,64 \\
\hline R45 & D. z. & 1,3 & 2,1 & 0,6 & 0,5 & 1,5 & 1,9 & 0,5 & 0,6 & 0,6 & 1,8 & 1,9 & 2,2 & 0,6 & 0,6 & 0,7 & 0,1 & 5,26 \\
\hline R5 & D. $t$. & 1,1 & 1,7 & 0,3 & 0,5 & 1,6 & 2,0 & 0,5 & 0,2 & 0,4 & 1,4 & 1,5 & 2,0 & 0,6 & 0,2 & 1,4 & 0,8 & 4,96 \\
\hline R6 & D. $t$. & 1,5 & 2,0 & 0,5 & 0,3 & 1,5 & 1,9 & 0,5 & 0,7 & 0,3 & 1,8 & 1,3 & 1,8 & 0,6 & 0,4 & 1,8 & 1,2 & 5,22 \\
\hline R7 & D. c. & 0,9 & 1,6 & 0,3 & 0,5 & 1,4 & 1,3 & 0,5 & 0,2 & 0,4 & 1,6 & 1,2 & 1,4 & 0,4 & 0,3 & 1,8 & 1,4 & 5,39 \\
\hline R8 & D. C. & 0,9 & 1,9 & 0,3 & 0,7 & 1,2 & 2,0 & 0,8 & 0,7 & 0,3 & 1,9 & 1,1 & 1,9 & 0,7 & 0,6 & 2,1 & 1,3 & 5,62 \\
\hline R9 & D. $a$. & 0,9 & 1,6 & 0,6 & 0,3 & 1,3 & 1,7 & 0,3 & $-0,3$ & 1,1 & 2,4 & 1,5 & 1,3 & 0,5 & 0,3 & 1,9 & 1,3 & 7,28 \\
\hline RAK & D. $a$. & 2,5 & 2,2 & 0,8 & $-0,1$ & 2,2 & 2,1 & 0,7 & 0,0 & 0,5 & 2,5 & 1,9 & 2,3 & 0,9 & 0,4 & 2,4 & 1,5 & 7,54 \\
\hline RAMZ01 & D. o. & 2,0 & 3,0 & 0,5 & 1,0 & 2,3 & 2,5 & 0,7 & 0,3 & 0,6 & 1,7 & 1,8 & 2,5 & 0,7 & 0,8 & 1,9 & 2,1 & 6,32 \\
\hline RAMZ02 & D.t. & 1,5 & 2,4 & 0,4 & 0,7 & 1,5 & 2,2 & 0,4 & 0,6 & 0,4 & 1,1 & 1,4 & 2,4 & 0,5 & 0,9 & 1,3 & 1,3 & 5,07 \\
\hline RAMZ03 & D. C. & 1,4 & 3,7 & 0,6 & 2,5 & 1,3 & 3,4 & 0,6 & 2,0 & 0,5 & 1,9 & 1,2 & 2,6 & 0,5 & 1,3 & 2,0 & 1,9 & 5,53 \\
\hline RAMZ04 & D. $a$. & 2,0 & 1,6 & 0,7 & $-0,3$ & 2,0 & 1,5 & 0,6 & $-0,3$ & 0,7 & 2,2 & 1,7 & 1,6 & 0,8 & $-0,5$ & 2,0 & 2,0 & 7,44 \\
\hline RAMZ06 & D. C. & 1,5 & 3,5 & 0,5 & 1,9 & 1,5 & 3,0 & 0,7 & 1,6 & 0,7 & 2,1 & 1,5 & 2,9 & 0,7 & 1,4 & 2,2 & 2,3 & 5,47 \\
\hline RAMZ07 & D. C. & 1,1 & 4,1 & 0,3 & 3,0 & 1,6 & 3,5 & 0,9 & 2,0 & 0,8 & 2,4 & 1,5 & 3,8 & 0,4 & 2,1 & 2,3 & 2,3 & 5,70 \\
\hline RAMZ08 & D.t. & 1,6 & 1,7 & 0,4 & 0,1 & 1,7 & 1,9 & 0,4 & 0,1 & 0,4 & 1,2 & 1,5 & 1,7 & 0,6 & 0,2 & 1,5 & 1,6 & 4,98 \\
\hline RAMZ09 & D. z. & 1,6 & 2,4 & 0,6 & 0,9 & 1,7 & 2,4 & 0,7 & 0,8 & 0,6 & 1,9 & 1,7 & 2,6 & 0,8 & 0,6 & 1,8 & 2,0 & 5,30 \\
\hline RAMZ10 & D. i. & 2,2 & 2,7 & 0,8 & 0,5 & 2,3 & 2,2 & 0,8 & 0,6 & 0,5 & 2,1 & 2,0 & 2,6 & 0,8 & 0,6 & 2,0 & 2,0 & 6,41 \\
\hline RAMZ11 & D. $i$. & 2,1 & 2,5 & 0,7 & 0,6 & 2,3 & 2,8 & 0,8 & 0,4 & 0,6 & 2,3 & 1,9 & 2,1 & 0,8 & 0,9 & 2,2 & 2,2 & 6,41 \\
\hline RAMZ13 & D.t. & 1,5 & 2,4 & 0,6 & 0,9 & 1,8 & 2,0 & 0,3 & 0,5 & 0,3 & 0,9 & 1,4 & 2,5 & 0,7 & 0,9 & 1,1 & 1,2 & 4,98 \\
\hline RYŽ 1 & D. C. & 1,5 & 3,6 & 0,6 & 2,1 & 2,0 & 3,3 & 0,9 & 1,7 & 0,8 & 1,9 & 1,6 & 3,1 & 0,8 & 2,0 & 2,0 & 1,3 & 5,42 \\
\hline RYŽ 2 & D. $a$. & 1,8 & 2,3 & 0,4 & 0,4 & 1,8 & 2,2 & 0,7 & 0,5 & 0,5 & 1,8 & 2,0 & 2,4 & 0,7 & 0,4 & 2,0 & 1,2 & 7,12 \\
\hline RYŽ 3 & D. $a$. & 1,8 & 2,1 & 0,5 & 0,4 & 1,8 & 1,9 & 0,7 & 0,2 & 0,5 & 1,9 & 1,5 & 2,0 & 0,6 & 0,4 & 0,7 & 0,1 & 7,20 \\
\hline SRN & D. i. & 2,5 & 4,0 & 0,7 & 1,3 & 2,3 & 3,5 & 0,9 & 1,5 & 0,8 & 2,3 & 2,1 & 3,9 & 0,9 & 1,9 & 2,8 & 1,9 & 6,12 \\
\hline SRN 01 & D. i. & 2,2 & 2,9 & 0,4 & 0,9 & 2,1 & 2,8 & 0,8 & 1,0 & 0,5 & 2,2 & 2,0 & 2,6 & 0,6 & 0,6 & 2,3 & 2,4 & 6,08 \\
\hline SRN 02 & D. $i$. & 2,4 & 3,0 & 0,5 & 0,7 & 2,2 & 3,0 & 0,8 & 0,8 & 0,5 & 1,2 & 2,0 & 3,2 & 0,8 & 0,9 & 2,5 & 2,6 & 6,08 \\
\hline STA 1 & D. o. & 1,9 & 2,5 & 0,5 & 0,5 & 2,1 & 2,3 & 0,7 & 0,3 & 0,7 & 1,8 & 1,8 & 2,0 & 0,6 & 0,4 & 2,3 & 1,7 & 6,40 \\
\hline STA 2 & D. o. & 2,2 & 2,5 & 0,7 & 0,4 & 2,4 & 3,1 & 1,0 & 0,3 & 0,6 & 2,2 & 1,8 & 2,3 & 0,8 & 0,8 & 2,2 & 1,4 & 6,49 \\
\hline Š1 & D. $a$. & 1,8 & 2,0 & 0,4 & 0,1 & 1,9 & 2,2 & 0,6 & 0,4 & 0,5 & 1,7 & 1,6 & 1,9 & 0,6 & 0,4 & 1,4 & 0,8 & 7,09 \\
\hline Š10 & D. $a$. & 2,3 & 1,6 & 0,6 & $-0,7$ & 1,7 & 1,6 & 0,6 & $-0,5$ & 0,8 & 2,2 & 1,9 & 1,4 & 0,9 & $-0,6$ & 2,3 & 1,4 & 7,28 \\
\hline Š11 & D. $a$. & 2,1 & 2,1 & 0,8 & $-0,4$ & 2,2 & 1,9 & 0,5 & $-0,1$ & 1,1 & 2,3 & 2,2 & 2,2 & 0,7 & 0,4 & 2,1 & 1,5 & 7,28 \\
\hline Š2 & D. i. & 1,6 & 2,8 & 0,6 & 1,4 & 1,9 & 3,0 & 0,8 & 1,1 & 0,7 & 2,3 & 1,8 & 3,1 & 0,9 & 1,2 & 2,0 & 1,2 & 6,37 \\
\hline Š4 & D. $a$. & 2,2 & 2,7 & 0,8 & 0,2 & 2,3 & 2,1 & 0,8 & 0,4 & 0,7 & 2,5 & 1,9 & 2,4 & 0,8 & 0,2 & 2,5 & 1,7 & 7,30 \\
\hline Š5 & D. $a$. & 1,9 & 2,8 & 0,5 & 0,5 & 2,1 & 3,0 & 0,6 & 1,1 & 0,4 & 1,8 & 2,3 & 1,9 & 0,7 & $-0,2$ & 1,8 & 1,2 & 7,19 \\
\hline Š6 & D. $a$. & 2,1 & 2,2 & 0,7 & 0,2 & 2,1 & 2,4 & 0,7 & 0,5 & 0,6 & 2,3 & 1,8 & 1,8 & 0,7 & 0,4 & 1,1 & 0,4 & 7,31 \\
\hline ธ̌7 & D. i. & 1,8 & 1,9 & 0,5 & $-0,2$ & 1,6 & 1,7 & 0,6 & 0,2 & 0,5 & 1,5 & 1,5 & 1,7 & 0,7 & 0,3 & 1,7 & 1,0 & 6,34 \\
\hline Š8 & D. $a$. & 2,0 & 1,5 & 0,7 & $-0,5$ & 2,1 & 1,7 & 0,6 & $-0,5$ & 0,8 & 2,1 & 1,4 & 1,4 & 0,8 & 0,4 & 1,8 & 1,0 & 6,90 \\
\hline Š8 & D. $a$. & 1,7 & 1,4 & 0,4 & $-0,3$ & 1,9 & 1,4 & 0,5 & $-0,1$ & 0,7 & 1,9 & 1,6 & 1,1 & 0,5 & 0,1 & 1,8 & 1,2 & 6,90 \\
\hline Š9 & D. $a$. & 2,4 & 3,5 & 0,9 & 1,4 & 2,1 & 3,7 & 0,9 & 1,5 & 0,8 & 2,9 & 2,2 & 3,1 & 0,8 & 1,0 & 1,6 & 0,8 & 7,33 \\
\hline T1 & D. C. & 1,6 & 2,3 & 0,4 & 0,4 & 2,0 & 2,0 & 0,6 & 0,5 & 0,6 & 2,5 & 1,9 & 2,3 & 0,4 & 0,4 & 1,7 & 1,2 & 5,56 \\
\hline T10 & D. $t$. & 1,5 & 2,5 & 0,5 & 0,6 & 1,4 & 2,5 & 0,4 & 0,9 & 0,3 & 0,9 & 1,3 & 2,4 & 0,6 & 0,7 & 1,4 & 0,7 & 5,01 \\
\hline
\end{tabular}




\begin{tabular}{|c|c|c|c|c|c|c|c|c|c|c|c|c|c|c|c|c|c|c|}
\hline $\mathrm{T} 2$ & D. $t$. & 1,4 & 1,9 & 0,4 & 0,6 & 1,4 & 2,2 & 0,4 & 0,9 & 0,2 & 1,3 & 1,5 & 2,4 & 0,5 & 0,4 & 1,4 & 0,8 & 5,00 \\
\hline T3 & D. $t$. & 1,5 & 2,3 & 0,3 & 0,9 & 1,4 & 2,6 & 0,4 & 1,0 & 0,4 & 0,8 & 1,8 & 2,9 & 0,6 & 1,9 & 1,4 & 0,8 & 5,02 \\
\hline T4 & D.t. & 1,7 & 2,0 & 0,4 & 0,4 & 1,4 & 1,7 & 0,4 & 0,3 & 0,5 & 1,4 & 1,4 & 2,3 & 0,5 & 0,6 & 1,5 & 0,9 & 5,28 \\
\hline T5 & D. c. & 1,3 & 3,2 & 0,4 & 1,9 & 1,5 & 2,8 & 0,5 & 1,4 & 0,5 & 2,3 & 1,5 & 2,0 & 0,7 & 0,3 & 1,7 & 0,9 & 5,73 \\
\hline T6 & D. z. & 1,7 & 3,2 & 0,4 & 1,4 & 1,6 & 3,0 & 0,5 & 1,5 & 0,5 & 1,5 & 1,5 & 3,0 & 0,7 & 1,6 & 1,9 & 1,2 & 5,40 \\
\hline T7 & D. $t$. & 1,4 & 2,0 & 0,5 & 1,2 & 1,3 & 2,7 & 0,3 & 1,4 & 0,3 & 1,1 & 1,6 & 2,6 & 0,7 & 0,7 & 1,3 & 0,7 & 5,12 \\
\hline T8 & D. t. & 1,5 & 2,3 & 0,3 & 0,8 & 1,5 & 2,3 & 0,4 & 1,1 & 0,3 & 0,9 & 1,7 & 2,3 & 0,5 & 0,8 & 1,3 & 0,8 & 5,09 \\
\hline T9 & D. C. & 1,5 & 3,3 & 0,5 & 1,3 & 1,5 & 2,9 & 0,5 & 1,3 & 0,5 & 1,0 & 1,6 & 3,1 & 0,8 & 1,3 & 1,5 & 0,7 & 5,28 \\
\hline TET 01 & D. $a$. & 1,9 & 2,1 & 0,6 & $-0,1$ & 1,9 & 2,1 & 0,6 & 0,1 & 0,5 & 2,2 & 2,0 & 1,8 & 0,8 & $-0,1$ & 2,5 & 2,6 & 7,36 \\
\hline TET 02 & D. $a$. & 1,5 & 1,4 & 0,5 & $-0,2$ & 2,1 & 1,1 & 0,5 & $-0,3$ & 0,6 & 2,4 & 1,7 & 1,6 & 0,8 & $-0,3$ & 2,2 & 2,4 & 7,23 \\
\hline TET 03 & D. $a$. & 2,0 & 1,9 & 0,7 & $-0,3$ & 1,8 & 1,7 & 0,4 & 0,1 & 0,7 & 2,2 & 1,6 & 1,7 & 0,8 & $-0,1$ & 2,7 & 2,8 & 7,24 \\
\hline TET 04 & D. $a$. & 1,8 & 1,5 & 0,7 & $-0,3$ & 1,8 & 1,4 & 0,5 & $-0,3$ & 0,6 & 2,0 & 1,7 & 1,4 & 0,8 & $-0,2$ & 2,1 & 2,1 & 7,29 \\
\hline VCE 01 & D.c. & 1,1 & 2,7 & 0,4 & 2,3 & 1,6 & 2,7 & 0,9 & 1,4 & 0,7 & 1,9 & 1,8 & 3,3 & 0,6 & 0,8 & 1,9 & 2,1 & 5,62 \\
\hline VCE 02 & D. c. & 1,2 & 2,6 & 0,5 & 1,5 & 1,5 & 3,0 & 1,0 & 1,4 & 0,8 & 2,0 & 1,8 & 3,0 & 0,7 & 1,0 & 2,1 & 2,2 & 5,54 \\
\hline VCE 03 & D. $i$. & 1,5 & 2,5 & 0,5 & 0,8 & 1,8 & 2,2 & 0,8 & 0,6 & 0,7 & 2,0 & 1,7 & 3,1 & 0,7 & 1,1 & 1,9 & 2,0 & 6,40 \\
\hline VCE 04 & D. $i$. & 1,4 & 2,9 & 0,5 & 1,3 & 2,1 & 2,5 & 0,7 & 1,2 & 0,7 & 2,2 & 1,6 & 3,1 & 0,9 & 1,7 & 2,2 & 2,5 & 6,35 \\
\hline VCE1 & D. C. & 0,8 & 3,1 & 0,5 & 1,8 & 1,4 & 2,9 & 0,8 & 1,6 & 0,8 & 2,4 & 1,3 & 3,1 & 0,6 & 1,6 & 3,0 & 2,3 & 5,71 \\
\hline VCE2 & D. $i$. & 1,6 & 3,6 & 0,6 & 1,7 & 2,0 & 3,1 & 1,0 & 0,9 & 0,7 & 2,5 & 1,9 & 3,0 & 0,9 & 1,4 & 3,2 & 2,2 & 6,24 \\
\hline VJ1 & D. C. & 0,9 & 3,3 & 0,3 & 2,1 & 1,9 & 3,1 & 0,6 & 1,6 & 0,9 & 4,0 & 1,1 & 2,6 & 0,4 & 1,4 & 1,3 & 0,9 & 5,55 \\
\hline VJ2 & D. c. & 1,0 & 3,3 & 0,4 & 1,9 & 1,9 & 3,3 & 0,9 & 1,7 & 0,8 & 3,6 & 1,3 & 2,5 & 0,5 & 1,2 & 1,4 & 0,9 & 5,48 \\
\hline VJ3 & D. C. & 0,9 & 2,6 & 0,3 & 1,4 & 1,6 & 2,1 & 0,9 & 1,6 & 0,8 & 3,2 & 1,3 & 2,2 & 0,4 & 0,6 & 1,4 & 1,1 & 5,35 \\
\hline VJ4 & D. c. & 1,2 & 2,6 & 0,5 & 1,3 & 1,8 & 2,6 & 0,7 & 1,4 & 0,8 & 2,9 & 1,4 & 2,5 & 0,5 & 0,9 & 2,4 & 1,9 & 5,49 \\
\hline VJ5 & D. c. & 1,3 & 3,1 & 0,4 & 1,7 & 2,0 & 3,1 & 1,0 & 1,0 & 0,8 & 2,9 & 1,1 & 2,9 & 0,7 & 1,6 & 1,9 & 1,2 & 5,50 \\
\hline VLT 01 & D. $a$. & 1,8 & 1,9 & 0,7 & 0,2 & 1,9 & 2,0 & 0,9 & 0,1 & 0,6 & 2,7 & 1,9 & 2,5 & 0,9 & 0,5 & 2,7 & 2,8 & 7,19 \\
\hline VLT 02 & D. $a$. & 1,5 & 1,7 & 0,5 & 0,0 & 1,9 & 1,4 & 0,8 & $-0,2$ & 0,6 & 1,9 & 2,0 & 1,5 & 0,6 & $-0,4$ & 1,9 & 1,9 & 7,15 \\
\hline VLT 03 & D. $a$. & 1,6 & 1,8 & 0,7 & 0,0 & 1,9 & 1,9 & 0,7 & $-0,1$ & 0,6 & 2,5 & 1,9 & 1,7 & 0,7 & 0,1 & 2,1 & 2,2 & 7,37 \\
\hline VLT 04 & D. $i$. & 1,2 & 3,3 & 0,4 & 2,3 & 2,4 & 3,2 & 1,0 & 1,1 & 0,7 & 2,5 & 2,3 & 3,5 & 0,7 & 1,0 & 1,1 & 1,3 & 6,41 \\
\hline VLT 05 & D. $a$. & 2,3 & 2,7 & 0,7 & 0,4 & 2,4 & 2,8 & 0,9 & 0,5 & 0,8 & 2,0 & 2,0 & 2,5 & 0,8 & 0,4 & 1,0 & 1,0 & 7,20 \\
\hline VLT 06 & D. $i$. & 1,7 & 3,6 & 0,6 & 1,7 & 2,4 & 3,9 & 1,1 & 1,4 & 0,7 & 2,6 & 1,8 & 3,9 & 0,8 & 1,6 & 2,4 & 2,5 & 6,31 \\
\hline VLT 07 & D. c. & 1,4 & 4,5 & 0,5 & 2,6 & 2,3 & 4,6 & 1,0 & 2,5 & 1,0 & 3,1 & 1,3 & 4,4 & 0,4 & 2,6 & 1,4 & 1,4 & 5,91 \\
\hline VLT 08 & D. $i$. & 1,8 & 4,8 & 0,6 & 2,5 & 2,8 & 4,0 & 1,1 & 1,3 & 0,8 & 3,0 & 2,5 & 4,8 & 0,9 & 2,8 & 2,9 & 3,1 & 6,31 \\
\hline VÝSL 1 & D.c. & 1,0 & 2,6 & 0,6 & 1,2 & 1,5 & 2,0 & 0,5 & 0,8 & 0,7 & 1,8 & 1,6 & 2,3 & 0,6 & 0,7 & 2,1 & 1,5 & 5,32 \\
\hline VYSL 2 & D. c. & 1,2 & 3,0 & 0,5 & 1,7 & 1,8 & 2,6 & 0,8 & 0,7 & 0,7 & 2,3 & 1,5 & 3,0 & 0,8 & 1,4 & 2,3 & 1,5 & 5,43 \\
\hline VÝSL 3 & D. C. & 1,0 & 2,9 & 0,5 & 1,2 & 1,9 & 2,5 & 0,8 & 0,5 & 0,8 & 2,2 & 1,6 & 2,9 & 0,9 & 1,1 & 1,9 & 1,0 & 5,36 \\
\hline Z1 & D. $a$. & 1,4 & 1,2 & 0,6 & $-0,1$ & 1,5 & 1,0 & 0,0 & 0,7 & 0,7 & 2,0 & 0,8 & 1,3 & 0,7 & 0,2 & 1,7 & 1,0 & 7,43 \\
\hline $\mathrm{Z2}$ & D. $a$. & 1,0 & 2,0 & 0,7 & 0,0 & 1,9 & 1,9 & 0,0 & 0,5 & 0,5 & 2,3 & 1,0 & 1,8 & 0,8 & 0,6 & 1,6 & 0,8 & 7,31 \\
\hline Z3 & D. $a$. & 1,7 & 2,2 & 0,5 & 0,6 & 2,2 & 2,5 & 0,0 & 0,2 & 0,8 & 2,4 & 1,1 & 2,2 & 0,6 & 0,6 & 1,7 & 1,1 & 7,50 \\
\hline Z4 & D. $a$. & 1,2 & 2,0 & 0,6 & 0,5 & 1,7 & 1,6 & 0,0 & 0,4 & 0,6 & 2,1 & 1,4 & 1,6 & 0,7 & 0,3 & 1,6 & 0,9 & 7,31 \\
\hline Z5 & D. $a$. & 1,4 & 2,4 & 0,7 & 0,5 & 2,1 & 2,3 & 0,0 & 0,2 & 0,7 & 2,6 & 1,4 & 1,9 & 0,8 & 0,6 & 1,9 & 1,0 & 7,39 \\
\hline Z6 & D. $a$. & 1,2 & 1,7 & 0,8 & 0,3 & 1,9 & 1,8 & 0,0 & 0,3 & 0,8 & 2,7 & 1,4 & 2,0 & 0,9 & 0,7 & 1,9 & 1,0 & 7,44 \\
\hline ZAD1 & D. $a$. & 2,2 & 2,7 & 0,8 & 0,1 & 2,7 & 2,8 & 1,0 & $-0,1$ & 0,6 & 2,8 & 2,2 & 2,5 & 1,0 & 0,6 & 3,2 & 2,2 & 7,20 \\
\hline ZAD2_1 & D. $i$. & 2,4 & 3,3 & 0,8 & 0,8 & 2,7 & 3,2 & 3,6 & 0,7 & 0,7 & 2,6 & 2,4 & 3,4 & 1,0 & 0,8 & 2,8 & 1,8 & 6,38 \\
\hline ZAD2_10 & D. $i$. & 2,1 & 2,7 & 0,7 & 0,6 & 2,3 & 2,7 & 0,8 & 0,6 & 0,5 & 2,3 & 1,6 & 2,7 & 0,8 & 0,7 & 2,1 & 1,4 & 6,27 \\
\hline ZAD2_11 & D. c. & 0,9 & 2,2 & 0,5 & 1,0 & 1,4 & 2,0 & 0,7 & 0,8 & 0,6 & 2,0 & 1,3 & 2,0 & 0,8 & 0,9 & 2,0 & 1,1 & 5,65 \\
\hline ZAD2_12 & D. $i$. & 1,9 & 2,1 & 0,5 & 0,3 & 1,9 & 2,1 & 0,7 & 0,2 & 0,5 & 1,9 & 1,5 & 2,2 & 0,8 & 0,2 & 1,9 & 1,1 & 6,36 \\
\hline ZAD2_2 & D. $i$. & 2,7 & 3,6 & 0,7 & 0,9 & 2,8 & 3,3 & 1,1 & 0,9 & 0,8 & 3,2 & 2,4 & 3,4 & 0,9 & 1,2 & 3,5 & 2,6 & 6,28 \\
\hline ZAD2_3 & & 2,3 & 1,9 & 0,8 & $-0,3$ & 2,0 & 2,1 & 0,6 & $-0,1$ & 0,6 & 2,3 & 2,6 & 2,2 & 0,8 & 0,1 & 2,3 & 1,5 & 7,19 \\
\hline ZAD2_4 & D. $i$. & 1,9 & 2,8 & 0,6 & 0,7 & 2,0 & 2,5 & 0,8 & 0,6 & 0,7 & 2,2 & 1,8 & 2,4 & 0,9 & 0,3 & 2,5 & 1,6 & 6,48 \\
\hline ZAD2_5 & D. $i$. & 2,1 & 2,5 & 0,8 & 0,3 & 2,1 & 2,2 & 2,4 & 0,2 & 0,7 & 2,1 & 1,9 & 2,8 & 0,9 & 0,8 & 2,2 & 1,3 & 6,35 \\
\hline ZAD2_6 & D. C. & 0,9 & 2,8 & 0,5 & 1,4 & 1,7 & 2,0 & 0,7 & 0,7 & 0,5 & 2,1 & 1,6 & 2,8 & 0,7 & 1,7 & 2,3 & 1,5 & 5,67 \\
\hline
\end{tabular}




\begin{tabular}{|c|c|c|c|c|c|c|c|c|c|c|c|c|c|c|c|c|c|c|}
\hline ZAD2_7 & D. $a$. & 2,4 & 2,5 & 0,6 & 0,3 & 2,6 & 3,1 & 0,9 & 0,5 & 0,7 & 3,2 & 2,3 & 3,3 & 0,8 & 0,6 & 2,1 & 1,3 & 7,11 \\
\hline ZAD2_8 & D. c. & 1,1 & 2,8 & 0,5 & 1,8 & 1,6 & 2,3 & 0,7 & 1,0 & 0,7 & 2,1 & 1,3 & 2,8 & 0,7 & 1,2 & 2,3 & 1,7 & 5,46 \\
\hline ZAD2_9 & D. $i$ & 1,9 & 2,3 & 0,8 & 0,2 & 2,0 & 2,5 & 1,0 & 0,4 & 0,7 & 2,2 & 2,0 & 2,7 & 0,9 & 0,4 & 2,1 & 1,2 & 6,33 \\
\hline ZLI 01 & D. i. & 1,4 & 2,5 & 0,5 & 1,1 & 2,0 & 2,6 & 1,1 & 1,0 & 0,6 & 2,4 & 1,5 & 2,6 & 0,6 & 0,7 & 2,0 & 2,1 & 6,21 \\
\hline ZLI 02_1 & D. $t$. & 1,9 & 2,9 & 0,5 & 1,1 & 1,8 & 2,2 & 0,5 & 0,1 & 0,5 & 1,2 & 1,4 & 2,1 & 0,4 & 0,5 & 1,5 & 1,5 & 5,21 \\
\hline ZLI 02_2 & D. z. & 1,4 & 2,0 & 0,4 & 0,6 & 1,6 & 2,0 & 0,6 & 0,4 & 0,5 & 1,3 & 1,7 & 2,1 & 0,6 & 0,9 & 1,8 & 1,9 & 5,17 \\
\hline ZLI 03 & D. c. & 1,4 & 3,0 & 0,3 & 1,4 & 1,7 & 3,2 & 0,6 & 1,6 & 0,7 & 1,6 & 1,5 & 3,1 & 0,6 & 1,5 & 1,8 & 2,0 & 5,32 \\
\hline ZLI 04 & D. z. & 1,7 & 3,4 & 0,5 & 1,5 & 2,0 & 3,4 & 0,6 & 1,4 & 0,6 & 1,3 & 2,1 & 3,5 & 0,7 & 1,3 & 1,6 & 1,6 & 5,15 \\
\hline ZLI 05 & D. z. & 1,5 & 1,9 & 0,4 & 0,2 & 1,6 & 1,6 & 0,5 & 0,1 & 0,5 & 1,3 & 1,5 & 1,9 & 0,4 & 0,5 & 1,5 & 1,5 & 5,17 \\
\hline ZLI 06 & D. o. & 2,2 & 2,7 & 0,6 & 0,3 & 2,0 & 2,5 & 0,7 & 0,6 & 0,5 & 2,3 & 2,0 & 2,7 & 0,9 & 0,6 & 2,2 & 2,4 & 6,19 \\
\hline ZLI 07 & D. z. & 1,1 & 2,3 & 0,4 & 1,2 & 1,5 & 1,1 & 0,5 & 1,1 & 0,5 & 1,1 & 1,2 & 2,7 & 0,4 & 1,3 & 1,4 & 1,5 & 5,16 \\
\hline ZLI 08 & D. $a$. & 2,1 & 2,4 & 0,7 & 0,0 & 2,2 & 2,5 & 0,8 & 0,1 & 0,9 & 3,4 & 1,8 & 2,2 & 1,0 & 0,7 & 2,0 & 2,2 & 7,14 \\
\hline ZLI 09_1 & D.t. & 1,1 & 2,5 & 0,4 & 1,1 & 1,4 & 2,4 & 0,6 & 1,2 & 0,4 & 1,4 & 1,4 & 2,8 & 0,4 & 1,2 & 1,5 & 1,5 & 5,19 \\
\hline ZLI 09_2 & D. $i$ & 1,1 & 2,5 & 0,5 & 2,7 & 1,6 & 2,3 & 0,6 & 1,2 & 0,6 & 2,2 & 1,9 & 2,5 & 0,7 & 1,0 & 1,8 & 1,9 & 6,38 \\
\hline ZLI 10 & D. z. & 1,3 & 2,1 & 0,4 & 0,8 & 1,5 & 2,1 & 0,7 & 0,6 & 0,5 & 1,1 & 1,5 & 2,1 & 0,5 & 0,4 & 1,5 & 1,6 & 5,20 \\
\hline ZLI 11 & D. $a$. & 1,8 & 2,4 & 0,7 & 0,0 & 1,8 & 2,2 & 0,9 & 0,2 & 0,8 & 2,4 & 1,9 & 1,8 & 0,7 & $-0,1$ & 1,7 & 1,8 & 7,32 \\
\hline ZLI 12 & D. $a$. & 1,8 & 2,7 & 0,4 & 1,1 & 2,0 & 3,0 & 1,0 & 0,5 & 0,8 & 0,6 & 1,5 & 2,7 & 0,4 & 0,7 & 2,1 & 2,1 & 7,61 \\
\hline ZLI 13 & D. o. & 2,0 & 2,8 & 0,6 & 0,7 & 2,0 & 2,6 & 0,6 & 0,5 & 0,6 & 1,5 & 1,5 & 2,7 & 0,7 & 1,4 & 1,8 & 1,9 & 6,02 \\
\hline ZLI 14 & D. c. & 0,8 & 2,5 & 0,4 & 1,5 & 1,2 & 2,4 & 0,7 & 1,1 & 0,6 & 1,9 & 1,3 & 2,6 & 0,5 & 1,3 & 1,8 & 1,9 & 5,52 \\
\hline ZLI 15_1 & D. z. & 1,2 & 3,0 & 0,5 & 1,5 & 1,4 & 3,0 & 0,6 & 1,5 & 0,6 & 1,2 & 1,5 & 2,9 & 0,7 & 1,0 & 1,7 & 1,8 & 5,21 \\
\hline ZLI 15_2 & D. $t$. & 1,7 & 3,1 & 0,3 & 1,8 & 1,6 & 3,1 & 0,5 & 1,1 & 0,5 & 1,4 & 1,4 & 2,4 & 0,5 & 0,9 & 1,5 & 1,6 & 5,13 \\
\hline ZMU 01 & D. $a$. & 1,9 & 1,6 & 0,7 & $-0,3$ & 1,5 & 1,7 & 0,8 & $-0,1$ & 0,8 & 2,0 & 1,9 & 1,4 & 0,8 & $-0,6$ & 2,1 & 2,2 & 7,25 \\
\hline ZMU 02 & D. $a$. & 2,4 & 2,3 & 0,6 & $-0,1$ & 2,1 & 2,3 & 0,7 & 0,2 & 0,8 & 2,2 & 2,0 & 2,3 & 0,8 & 0,4 & 1,4 & 1,5 & 7,19 \\
\hline
\end{tabular}

Northern Europe

\begin{tabular}{|c|c|c|c|c|c|c|c|c|c|c|c|c|c|c|c|c|c|c|}
\hline ID & taxa & v2 & v3 & v4 & v5 & v6 & v7 & v8 & v9 & v10 & v11 & v12 & v13 & v14 & v15 & v16 & v17 & GS (2C) \\
\hline FIN 1/1 & D. z. & 1,3 & 2,8 & 2,4 & 1,3 & 1,4 & 2,6 & 0,6 & 1,1 & 0,5 & 1,5 & 1,5 & 2,6 & 0,8 & 1,2 & 1,9 & 1,0 & 5,24 \\
\hline FIN $1 / 2$ & D. z. & 1,3 & 2,5 & 0,6 & 1,1 & 1,5 & 2,5 & 0,6 & 0,8 & 0,5 & 1,8 & 1,5 & 2,4 & 0,6 & 0,8 & 1,8 & 1,2 & 5,13 \\
\hline FIN 2 & D. c. & 1,4 & 2,1 & 0,5 & 0,7 & 1,7 & 2,5 & 0,6 & 0,8 & 0,5 & 2,1 & 1,6 & 2,6 & 0,6 & 0,7 & 2,0 & 1,3 & 5,36 \\
\hline FIN 3/1 & D. c. & 1,1 & 4,4 & 0,6 & 2,9 & 1,7 & 3,6 & 0,8 & 1,5 & 0,6 & 2,5 & 1,4 & 4,1 & 0,8 & 2,4 & 2,1 & 1,2 & 5,46 \\
\hline FIN $3 / 2$ & D. c. & 0,9 & 2,7 & 0,4 & 2,1 & 1,2 & 3,4 & 0,7 & 1,9 & 0,7 & 2,3 & 1,3 & 3,0 & 0,7 & 1,5 & 2,1 & 1,4 & 5,4 \\
\hline FIN 4/1 & D. c. & 1,0 & 3,0 & 0,5 & 1,8 & 1,5 & 2,8 & 0,8 & 1,3 & 0,5 & 1,9 & 1,5 & 2,9 & 0,9 & 1,6 & 2,0 & 1,1 & 5,42 \\
\hline $\mathrm{FIN} 4 / 2$ & D. c. & 1,1 & 2,4 & 0,5 & 1,1 & 1,4 & 2,4 & 0,6 & 0,9 & 0,6 & 1,5 & 1,3 & 2,6 & 0,9 & 0,6 & 1,8 & 0,9 & 5,22 \\
\hline FIN 5/1 & D. c. & 1,1 & 3,5 & 0,6 & 2,1 & 1,6 & 3,4 & 0,8 & 1,8 & 0,8 & 2,4 & 1,7 & 4,0 & 0,8 & 2,2 & 2,6 & 1,7 & 5,54 \\
\hline FIN 5/2 & D. C. & 5,3 & 3,1 & 0,5 & 1,6 & 1,9 & 3,2 & 0,9 & 1,6 & 0,9 & 2,9 & 1,4 & 3,0 & 0,8 & 1,4 & 2,8 & 2,0 & 5,49 \\
\hline FIN 5/3 & D. c. & 1,0 & 3,2 & 0,7 & 1,9 & 1,7 & 3,1 & 0,7 & 1,6 & 0,7 & 2,1 & 1,6 & 2,8 & 0,8 & 1,5 & 2,1 & 1,3 & 5,44 \\
\hline FIN 6/1 & D. c. & 1,5 & 2,0 & 0,6 & 0,4 & 1,4 & 2,0 & 0,6 & 0,3 & 0,5 & 1,5 & 1,1 & 1,6 & 0,9 & 0,6 & 1,4 & 0,5 & 5,47 \\
\hline FIN 6/2 & D. z. & 1,4 & 2,3 & 0,5 & 0,5 & 1,7 & 2,2 & 0,6 & 0,7 & 1,4 & 1,4 & 1,4 & 2,0 & 0,6 & 0,6 & 1,6 & 1,0 & 5,14 \\
\hline FIN 7/1 & D. c. & 1,2 & 2,2 & 0,6 & 0,9 & 1,7 & 2,2 & 0,7 & 0,8 & 0,6 & 2,0 & 1,4 & 2,3 & 0,8 & 0,8 & 2,2 & 1,3 & 5,4 \\
\hline FIN $7 / 2$ & D. c. & 1,0 & 2,0 & 0,5 & 1,0 & 1,4 & 2,1 & 0,7 & 0,7 & 0,6 & 2,1 & 1,2 & 2,2 & 0,8 & 0,6 & 2,1 & 1,3 & 5,31 \\
\hline FIN 8/1 & D. C. & 0,9 & 2,9 & 0,6 & 2,4 & 1,6 & 2,7 & 0,8 & 1,5 & 1,0 & 2,5 & 1,6 & 2,7 & 0,9 & 1,3 & 2,2 & 1,3 & 5,43 \\
\hline FIN 8/2 & D. c. & 1,1 & 3,2 & 0,6 & 2,1 & 1,6 & 3,2 & 0,8 & 1,9 & 1,0 & 2,6 & 1,5 & 3,0 & 1,0 & 1,4 & 2,6 & 1,6 & 5,44 \\
\hline GPS21 & D. $a$. & 2,3 & 2,8 & 0,8 & $-0,5$ & 2,9 & 2,2 & 0,5 & 0,5 & 1,6 & 2,1 & 2,9 & 2,1 & 1,1 & $-0,8$ & 1,9 & 0,8 & 7,12 \\
\hline NOR B/1 & D. $a$. & 1,9 & 1,8 & 0,8 & $-0,2$ & 1,7 & 1,7 & 0,5 & 0,1 & 0,7 & 1,6 & 2,5 & 2,1 & 1,0 & $-0,2$ & 2,0 & 1,1 & 7,12 \\
\hline NOR B/2 & D. $a$. & 1,9 & 2,0 & 1,0 & $-0,1$ & 1,3 & 1,7 & 0,5 & $-0,1$ & 0,6 & 1,7 & 1,6 & 2,0 & 0,8 & 0,3 & 2,0 & 1,2 & 7,22 \\
\hline NOR C/1 & D. c. & 1,1 & 2,9 & 0,6 & 1,8 & 1,8 & 2,8 & 0,7 & 1,3 & 0,6 & 2,3 & 1,7 & 3,0 & 0,8 & 1,3 & 2,1 & 1,3 & 5,61 \\
\hline NOR $C / 2$ & D. c. & 1,3 & 2,5 & 0,5 & 1,3 & 1,5 & 2,2 & 0,6 & 0,9 & 1,0 & 2,0 & 1,4 & 2,6 & 0,9 & 1,1 & 2,0 & 1,1 & 5,46 \\
\hline NOR $C / 3$ & D. c. & 1,0 & 2,5 & 0,5 & 2,5 & 1,5 & 2,3 & 0,6 & 0,8 & 0,6 & 2,0 & 1,4 & 2,4 & 0,9 & 1,1 & 1,9 & 0 & 5,47 \\
\hline NOR1 & D. $a$. & 2,4 & 2,2 & 0,8 & $-0,2$ & 2,4 & 2,5 & 0,8 & 0,1 & 0,7 & 2,5 & 1,9 & 2,2 & 0,9 & 0,2 & 2,2 & 1,4 & 7,11 \\
\hline
\end{tabular}




\begin{tabular}{|c|c|c|c|c|c|c|c|c|c|c|c|c|c|c|c|c|c|c|}
\hline NOR2 & D. $a$. & 2,1 & 1,5 & 0,6 & $-0,5$ & 1,9 & 1,5 & 0,5 & $-0,3$ & 0,7 & 2,0 & 1,9 & 1,5 & 0,8 & $-0,3$ & 2,0 & 1,2 & 7,29 \\
\hline SC1 & D. $a$. & 2,1 & 2,3 & 0,8 & $-0,5$ & 3,4 & 2,6 & 0,6 & $-0,1$ & 1,7 & 2,2 & 1,6 & 2,6 & 0,9 & $-0,7$ & 1,9 & 1,0 & 7,07 \\
\hline SC10 & D. $a$. & 2,2 & 2,2 & 1,0 & $-0,4$ & 1,9 & 2,2 & 0,4 & $-0,5$ & 1,2 & 2,2 & 1,6 & 2,0 & 0,9 & $-0,7$ & 1,9 & 1,0 & 7,04 \\
\hline SC2 & D. $a$. & 3,4 & 2,8 & 1,0 & $-0,9$ & 3,6 & 2,8 & 0,5 & $-0,8$ & 1,2 & 1,9 & 3,3 & 2,9 & 1,1 & $-1,0$ & 1,9 & 0,9 & 7,06 \\
\hline SC3 & D. $a$. & 1,1 & 1,3 & 0,8 & $-0,7$ & 1,8 & 1,3 & 0,3 & $-0,4$ & 1,3 & 2,4 & 2,6 & 1,4 & 0,8 & $-1,2$ & 2,2 & 1,4 & 1 \\
\hline $\mathrm{SC} 4$ & D. a. & 2,4 & 2,6 & 0,8 & $-0,2$ & 1,9 & 2,0 & 0,8 & $-0,2$ & 0,7 & 2,1 & 2,9 & 2,2 & 0,8 & $-0,7$ & 2,4 & 1,5 & ,03 \\
\hline SC5 & D. c. & 0,8 & 2,1 & 0,2 & 1,1 & 1,6 & 2,4 & 0,5 & 0,9 & 0,7 & 2,8 & 1,1 & 2,2 & 0,7 & $-0,4$ & 1,9 & 1,2 & 5,43 \\
\hline SC6/1 & D. C. & 1,4 & 3,5 & 0,8 & 1,9 & 1,8 & 4,2 & 1,0 & 2,7 & 1,3 & 2,2 & 1,8 & 3,9 & 1,1 & $-1,4$ & 2,8 & 1,7 & 5,49 \\
\hline $\mathrm{SC} 6 / 2$ & D. c. & 1,2 & 2,3 & 0,5 & 1,3 & 2,1 & 2,6 & 0,8 & 0,8 & 0,9 & 2,5 & 0,9 & 2,7 & 0,8 & $-1,5$ & 2,3 & 1,6 &, 51 \\
\hline $\mathrm{SC6} / 3$ & D. c. & 1,9 & 2,9 & 0,5 & 1,2 & 1,8 & 2,4 & 0,7 & 0,9 & 1,2 & 2,5 & 1,2 & 2,8 & 0,7 & $-1,3$ & 2,1 & 1,4 & 49 \\
\hline SC6/4 & D. C. & 1,4 & 2,7 & 0,6 & 0,8 & 2,0 & 2,3 & 0,8 & 0,8 & 0,9 & 3,0 & 1,2 & 2,3 & 1,0 & $-1,3$ & 2,3 & 1,3 & 5,51 \\
\hline $\mathrm{SC} 6 / 5$ & D. C. & 1,6 & 3,4 & 0,7 & 1,4 & 1,7 & 3,9 & 0,8 & 1,9 & 0,6 & 2,3 & 1,4 & 3,3 & 0,8 & $-0,8$ & 2,0 & 1,2 & 5,43 \\
\hline sc6/6 & D. c. & 1,1 & 2,2 & 0,5 & 1,3 & 1,8 & 2,1 & 0,7 & $-0,4$ & 2,0 & 2,1 & 1,1 & 2,5 & 0,9 & $-1,2$ & 2,3 & 1,5 & 42 \\
\hline SC6/7 & D. C. & 1,0 & 2,1 & 0,4 & 1,1 & 1,9 & 2,5 & 0,6 & 1,1 & 0,7 & 2,1 & 1,4 & 2,2 & 0,7 & $-1,3$ & 1,8 & 1,0 & ויני \\
\hline SC6/8 & D. C. & 1,2 & 1,8 & 0,5 & 0,7 & 1,3 & 1,8 & 0,7 & 0,9 & 0,7 & 2,4 & 1,2 & 2,2 & 0,8 & $-1,5$ & 2,2 & 1,4 & 5,5 \\
\hline SC7 & D. $a$. & 1,2 & 1,5 & 0,8 & $-1,2$ & 2,1 & 1,4 & 0,3 & $-0,3$ & 0,9 & 2,0 & 1,4 & 1,1 & 0,8 & $-0,6$ & 2,0 & 1,3 & 7,08 \\
\hline SC8 & D. $a$. & 2,5 & 3,0 & 1,0 & $-0,9$ & 2,5 & 3,0 & 0,4 & 0,3 & 0,7 & 2,1 & 2,3 & 3,2 & 0,9 & $-0,9$ & 2,1 & 1,2 & 7,01 \\
\hline SC9 & D. $a$. & 2,6 & 2,6 & 0,8 & $-0,3$ & 2,9 & 2,4 & 0,3 & $-0,1$ & 1,1 & 2,1 & 1,7 & 2,6 & 0,8 & $-0,9$ & 2,0 & 1,2 & 6,98 \\
\hline SK $1 / 1$ & D. $a$. & 2,0 & 2,3 & 0,6 & 0,4 & 2,1 & 2,2 & 0,8 & 0,2 & 0,6 & 2,4 & 2,3 & 0,8 & 0,8 & 0,3 & 2,4 & 1,5 & 7,15 \\
\hline SK $1 / 2$ & D. $a$. & 2,2 & 2,7 & 0,8 & 0,1 & 2,4 & 2,4 & 0,8 & 0,1 & 0,8 & 2,6 & 2,5 & 0,9 & 0,9 & 0,5 & 2,4 & 1,5 & 7,26 \\
\hline SK $1 / 3$ & D. $a$. & 2,0 & 3,4 & 0,7 & 1,1 & 2,1 & 3,4 & 1,0 & 1,1 & 0,7 & 2,7 & 3,1 & 0,9 & 0,9 & 1,3 & 1,8 & 0,9 & ,28 \\
\hline SK 2/1 & D. $a$. & 2,3 & 1,8 & 0,6 & $-0,4$ & 2,0 & 1,7 & 0,7 & $-0,1$ & 0,6 & 2,4 & 1,8 & 0,9 & 0,9 & $-0,1$ & 2,3 & 1,4 & 7,18 \\
\hline SK $2 / 2$ & D. $a$. & 2,2 & 2,4 & 0,8 & $-0,2$ & 2,0 & 2,3 & 1,0 & 0,1 & 0,7 & 2,7 & 2,1 & 0,9 & 0,9 & 0,3 & 2,7 & 1,8 & 15 \\
\hline SK $3 / C$ & D. $a$. & 2,0 & 1,9 & 0,6 & 0,0 & 1,8 & 2,1 & 0,7 & 0,1 & 0,5 & 1,9 & 1,8 & 1,8 & 0,8 & 0,2 & 2,4 & 1,6 & 7,11 \\
\hline SK 3/D & D. $a$. & 2,4 & 2,0 & 0,8 & $-0,5$ & 1,8 & 2,1 & 0,8 & 0,0 & 0,7 & 2,7 & 1,6 & 2,0 & 0,8 & 0,1 & 2,7 & 2,0 & 7,06 \\
\hline SK 3A/1 & D. $a$. & 2,3 & 2,0 & 0,8 & $-0,3$ & 1,8 & 1,9 & 0,8 & $-0,1$ & 0,5 & 2,6 & 1,8 & 0,9 & 0,9 & $-0,1$ & 2,5 & 1,5 & 7,33 \\
\hline SK 3A/2 & D. $a$. & 2,2 & 2,0 & 0,6 & 0,2 & 2,0 & 1,6 & 0,6 & $-0,1$ & 0,7 & 2,0 & 1,7 & 1,7 & 0,9 & $-0,2$ & 2,2 & 1,4 & 32 \\
\hline SK $3 \mathrm{~A} / 3$ & D. $a$. & 2,2 & 2,4 & 0,8 & 0,9 & 2,1 & 2,8 & 0,6 & 0,8 & 0,6 & 1,6 & 1,8 & 3,2 & 0,8 & 0,9 & 1,4 & 0,6 & 2 \\
\hline SK 3B/1 & D. $a$. & 1,7 & 1,8 & 0,5 & 0,0 & 1,8 & 1,9 & 0,9 & 0,2 & 0,6 & 2,3 & 1,6 & 1,8 & 0,8 & 0,3 & 2,4 & 1,7 & ,12 \\
\hline SK 4/2 & D. $a$. & 2,4 & 2,7 & 0,7 & $-0,1$ & 2,5 & 2,4 & 1,1 & 0,1 & 0,7 & 2,5 & 2,0 & 2,4 & 1,0 & 0,5 & 2,6 & 1,6 & 7,28 \\
\hline SK $4 / 3$ & D. $a$. & 2,0 & 2,3 & 0,8 & $-0,1$ & 2,4 & 2,3 & 1,3 & 0,1 & 0,8 & 3,6 & 2,3 & 2,7 & 1,0 & $-0,1$ & 3,7 & 2,7 & 11 \\
\hline SK 4/4 & D. $a$. & 2,0 & 1,9 & 0,7 & $-0,1$ & 2,1 & 2,0 & 0,7 & $-0,1$ & 0,6 & 2,6 & 1,9 & 1,8 & 0,7 & $-0,2$ & 1,9 & 1,2 & 04 \\
\hline SK $4 / 5$ & D. $a$. & 2,6 & 3,7 & 0,5 & 0,7 & 2,3 & 3,3 & 1,2 & 0,8 & 0,8 & 2,8 & 2,1 & 3,5 & 0,9 & 1,2 & 3,3 & 2,4 & $r, 21$ \\
\hline VB & D. $a$. & 2,2 & 2,1 & 0,9 & $-0,1$ & 2,3 & 1,9 & 0,8 & 0,1 & 0,7 & 3,3 & 2,1 & 1,0 & 1,0 & $-0,6$ & 3,2 & 2,1 & 24 \\
\hline
\end{tabular}

